\title{
A well-posedness framework for inpainting based on coherence transport
}

\author{
by
}

Thomas März 



\title{
A WELL-POSEDNESS FRAMEWORK FOR INPAINTING BASED ON COHERENCE TRANSPORT
}

\author{
THOMAS MÄRZ *
}

\begin{abstract}
Image inpainting is the process of touching-up damaged or unwanted portions of a picture and is an important task in image processing. For this purpose Bornemann and März [J. Math. Imaging Vis. , 28 (2007), pp. 259278] introduced a very efficient method called Image Inpainting Based on Coherence Transport which fills the missing region by advecting the image information along integral curves of a coherence vector field from the boundary towards the interior of the hole. The mathematical model behind this method is a first-order functional advection PDE posed on a compact domain with all inflow boundary. We show that this problem is well-posed under certain conditions.
\end{abstract}

Key words. functional PDEs, method of characteristics, Lyapunov functions, functions of bounded variation, fixedpoint theory, image inpainting

AMS subject classifications. 26B30, 35F15, 35A30, 35D30, 47H10

1. Introduction. As image processing has become an active field of mathematical research $[15,5]$, the task of digital image inpainting has also been approached by mathematical methods in the last decades. Image inpainting serves the purpose of touching-up damaged or unwanted portions of a picture. In mathematical image processing, images are considered to be functions $w: \Omega_{0} \rightarrow \mathbb{R}$ defined on a typically rectangular image domain $\Omega_{0} \subset \mathbb{R}^{2}$. The value $w(x) \in \mathbb{R}$ often represents an intensity of light which is perceptible as a gray color. Technically, image inpainting is a problem of data interpolation: apart from $\Omega_{0}$ we are given, as part of the problem formulation, an inpainting domain, i.e., a subdomain $\Omega \subset \Omega_{0}$ which marks the damage or the portion which has to be touched-up. And the "good" part of the image, which is to be kept, is given as a function $u_{0}: \Omega_{0} \backslash \Omega \rightarrow \mathbb{R}$ defined on the data domain $\Omega_{0} \backslash \Omega\left(u_{0}\right.$ is undefined on $\Omega$ ). Now, the task is to find a function $u: \Omega \rightarrow \mathbb{R}$, defined on the missing part $\Omega$, which interpolates the data $u_{0}$. The very important side condition on an acceptable solution $u$ is that the completed image $\bar{u}:=u_{0} \cdot \mathbb{1}_{\Omega_{0} \backslash \Omega}+u \cdot \mathbb{1}_{\Omega}$, now defined on the whole image domain $\Omega_{0}$, should look nice, i.e., $u$ should interpolate the data $u_{0}$ in a visually plausible manner.

Since there is no ubiquitous mathematical definition of the terms "nice" and "visually plausible" such a definition is part of the task. Consequently, many different approaches to the inpainting problem have been made. Regarding non-texture inpainting PDE-based models were first suggested in the articles of Caselles et al. [14] or Masnou et al. [26] and several other PDE-based models have been proposed thereafter. These models were either phenomenologically derived, or came from variational principles, or were inspired by a variational idea with phenomenological modification of the Euler-Lagrange equation. The orders of the resulting PDEs range from first order, e.g. as in advection models [30,11,24], to second order, e.g. as in anisotropic diffusion [31] or the complex Ginzburg-Landau equation [21], to third order, e.g. as in nonlinear transport and curvature-driven diffusion equations [7, 16, 17], and fourth order, e.g. the models based on Euler's elastica [25, 28] or on a modified Cahn-Hilliard equation $[9,8,12]$. While PDE-based approaches are good at inpainting geometric features they are typically not as good at inpainting textures. In order to cope with textures exemplar-based and patch-based algorithms [18, 27, 29, 6] have been designed and combined with PDE models [13] as also non-local operators [20] have been developed to handle both textures and geometric features.

Many of the PDE-based approaches are non-linear and/or of a high order. Thus, the numerical algorithms are typically iterative and computationally expensive. In contrast, Image Inpainting Based on Coherence Transport [11, 24] features a first-order quasi-linear functional PDE. The resulting algorithm is very efficient because of the low order of the PDE (only one pass through the

\footnotetext{
* Oxford Centre for Collaborative Applied Mathematics, University of Oxford, OX1 3LB, UK. Email: maerz@maths . ox.ac.uk. This work was supported in part by the Graduiertenkolleg Angewandte Algorithmische Mathematik (GKAAM) funded by the Deutsche Forschungsgemeinschaft (DFG) at the Technische Universität München (TUM), and by award KUK-C1-013-04 made by King Abdullah University of Science and Technology (KAUST). Manuscript as of April 26, 2013.
} 


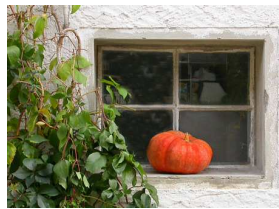

(a) original

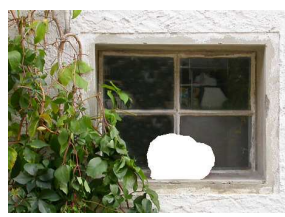

(b) masked

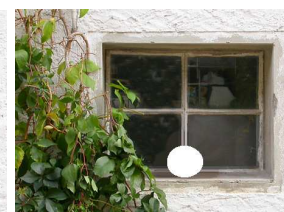

(c) stage 1

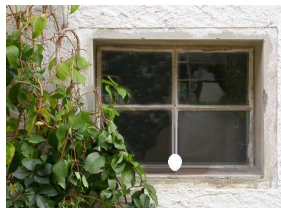

(d) stage 2

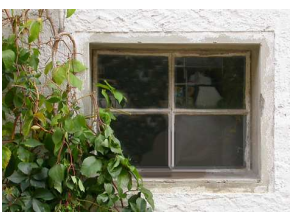

(e) inpainted

FIG. 1.1. Object removal by inpainting using the methods of [11, 24]. (a) shows the original image. (b) the masked region defines the inpainting domain. (c) $\mathcal{E}(d)$ show two intermediate stages of the inpainting process. (e) shows the final inpainted result.

pixels is required) while the functional dependence on the image makes for visually pleasing results. The aim of this paper is a framework for well-posedness of the functional PDE model underlying Image Inpainting Based on Coherence Transport [11, 24].

1.1. Image Inpainting Based on Coherence Transport. In the PDE-based approaches cited above, the authors usually start with continuous models and discretize them to obtain algorithms for digital image inpainting. In [11], we approached the problem from the opposite direction; our point of departure was the discrete inpainting problem.

The simple idea of the generic inpainting algorithm (cf. [11, Section 2]) is to fill the inpainting domain by traversing its pixels in a fixed order starting on the boundary and proceeding towards the interior. Thereby we compute the color-value of each pixel as a weighted average of colorvalues from initially known or already inpainted neighboring pixels.

In $[30,11]$ the default way to order the pixels was to order them according to their Euclidean distance-to-boundary. But generalized distance-to-boundary concepts can be applied too. Indeed, in [24] we have demonstrated that employing adapted distance functions produces more pleasing results. The algorithm is non-iterative, each pixel is visited once and the domain inpainted in a single pass. Figure 1.1 shows some intermediate stages of the filling process. Because of its simple structure the algorithm is extremely fast.

In [11] we demonstrated that this method is related to a PDE model. The high-resolution vanishing-viscosity limit (cf. [11, Section 3]) of the weighted-averaging step in the generic algorithm, for a special class of weights, yields the transport equation

$$
\langle c[u](x), \nabla u(x)\rangle=0, \quad x \in \Omega \backslash \Sigma,\left.\quad u\right|_{\Omega_{0} \backslash \Omega}=u_{0} .
$$

This is a PDE of first order where $c[u]$ defines characteristic curves along which we advect image information. In addition, in [11] we obtained that the transport field of (1.1) satisfies

$$
\langle c[u](x), \nabla T(x)\rangle \geq \beta>0, \quad x \in \Omega \backslash \Sigma
$$

where $T$ is exactly that generalized distance-to-boundary function (cf. [24]) which induced the pixel order in the algorithm. We will see later that this inequality rules out characteristic points, i.e., points where the characteristic curves would tangentially intersect the data domain.

Looking at (1.1), we can give the following rationale: imagine a (human) restorer doing brush strokes in the missing area $\Omega$. Assuming that she only uses color given by the data $u_{0}$ on $\partial \Omega$ and that her brush strokes lie along trajectories of a vector field $c$ which start at the boundary, we end up with the dynamical system

$$
\begin{array}{ll}
x^{\prime}=c[u](x), & x(0)=x_{0} \in \partial \Omega, \\
u^{\prime}=0, & u(0)=u_{0}\left(x_{0}\right),
\end{array}
$$

which describes exactly the characteristics of problem (1.1). Because we paint from every boundary point into $\Omega$, the characteristics, which are the brush strokes, are supposed to meet somewhere; the locations where they meet are contained in the exceptional set $\Sigma$ of equation (1.1). In fact control of this exceptional set is important for user interaction [24].

Ideally, in order to obtain an aesthetic inpainting, the vector field $c$ in (1.3) would need to reflect the full expertise of our restorer, which is generally not possible. At least the vector field 
$c$ should be adapted to and hence depend on the image $u$. In [11], the vector $c[u](x)$ includes an estimation of the tangent vector, which is tangent to the level line of $u$ going through the point $x$. This is because brush strokes are supposed to continue level lines of $u_{0}$ which have been interrupted by $\Omega$. By applying structure tensor analysis to the image we estimate approximate tangent information or so-called coherence information. The structure tensor $S$ is a positive semidefinite $2 \times 2$-matrix. Its set-up consists of the following two steps:

$$
v_{\sigma}(x, y)=\int_{\Omega_{0}} k_{\sigma}(x, y, h) u(h) d h, \quad S_{\sigma, \rho}(x)=\int_{\Omega_{0}} K_{\rho}(x, y) \nabla_{y} v_{\sigma}(x, y) \cdot \nabla_{y} v_{\sigma}(x, y)^{T} d y,
$$

where $\sigma>0$ and $\rho>0$ are the smoothing scales of the kernels $k_{\sigma}$ and $K_{\rho}$. The approximate tangent $g(x)$ is the eigenvector of $S_{\sigma, \rho}(x)$ with respect to the minimal eigenvalue. The motivation for this is (cf. [1]) that we look for a normed vector $g(x)$ which is almost orthogonal to the gradient $\nabla u$ in neighborhood of $x$, i.e., $\langle g(x), \nabla u\rangle^{2} \approx 0$. Since want to reduce the influence of noise and irrelevant small-scale features, we replace $u$ with a smoothed version $v_{\sigma}$ and consider $\left\langle g(x), \nabla_{y} v_{\sigma}\right\rangle^{2} \approx 0$ instead. Finally, we integrate the expression and formulate the almost orthogonality condition rigorously as a weighted least squares problem $\left(g^{T} S_{\sigma, \rho}(x) g \rightarrow \mathrm{min}\right)$ which involves the structure tensor. The benefit and the robustness of structure tensor analysis have been revealed in different works, e.g. [33] and [1].

The approximate tangent or coherence vector $g(x)$ then is inserted in the weight function

$$
w(x, y)=\frac{1}{|x-y|} \sqrt{\frac{\pi}{2}} \mu \exp \left(-\frac{\mu^{2}}{2 \varepsilon^{2}}\left\langle g^{\perp}(x), x-y\right\rangle^{2}\right)
$$

which is used in the algorithm to advect color-values by computing weighted averages [11]. The vector $g$ depends on the image $u$ and guides the advection as it influences $w$. The transport vector $c[u](x)$, as it corresponds to $w$ by the vanishing-viscosity limit (cf. [11, Section 3]), is thus guided by coherence and is mostly (up to regularization effects from vanishing-viscosity) aligned with the approximate tangent.

1.2. Contribution of this Paper. In the following we will provide a well-posedness framework for problem (1.1) which entails two major challenges: the general geometry which inpainting domains can exhibit on the one hand, and the involved dependence of $c[u]$ on $u$ on the other. Because of the structure tensor analysis, the vector $c[u](x)$ not only depends on the single value $u(x)$ but on a part $\left.u\right|_{B}$ of the image, meaning the dependence on $u$ is of a functional type.

Problem (1.1) is not restricted to inpainting or image processing and could occur in other contexts. In this paper, we study the problem independent of the particular construction of the coefficients within the inpainting model, but dependent on general features such as continuity, differentiability, etc. In fact we study the more general problem

$$
\begin{aligned}
\langle c[u](x), D u\rangle & =f[u](x) \cdot \mathcal{L}^{2}, \quad \text { in } \quad \Omega \backslash \Sigma, \\
\left.u\right|_{\partial \Omega} & =u_{0} .
\end{aligned}
$$

where $D u$ denotes the $B V$-derivative measure and $\mathcal{L}^{2}$ the Lebesgue measure.

Our strategy is to focus on the linear problem which we obtain from fixing the functional argument $c[v](x)$ by a function $v$ first. In this step we cope with the general geometry of inpainting domains $\Omega$ as well as that of the exceptional set $\Sigma$. By taking into account a regularization feature which is true for our inpainting model, namely that $c[v]$ is $C^{1}$ on $\Omega \backslash \Sigma$ for every choice of $v$, we construct a candidate solution using the method of characteristics. The characteristics start at the boundary and cover all of $\Omega \backslash \Sigma$ where we can now define a solution. But since $\Sigma$ is the place where different characteristics meet which might provide incompatible function values, we cannot expect the solution to be continuous across $\Sigma$, even in the case of smooth boundary data. In Lemma 3.5 we show that constructed solution can be extended onto the exceptional set $\Sigma$ as an element of the space $B V(\Omega)$ and we give an estimate of its $B V$-norm in terms of the problem data. 
Apart from the uniqueness of the solution (Theorem 3.8) we prove in Theorem 3.9 that the solution depends continuously, with respect to the $B V$-weak*-topology, on the coefficient functions, the boundary data, and also on the generalized distance-to-boundary function $T$.

In the second step we tackle the quasi-linear problem (1.6) by fixed-point theory. From the linear theory we obtain a solution $U[v]$ depending on $v$. Now, $U$ is a self-mapping (Corollary 4.2) and so any fixed-point $u=U[u]$ of the map $U$ will solve (1.6). Our existence result of Theorem 4.5 is a consequence of Schauder's fixed-point theorem and allows for a very general functional dependence of the coefficients in (1.6) on the solution. Theorem 4.5 as well as Schauder's theorem does not provide uniqueness. Indeed, we give an example which demonstrates that the solution is not unique when we allow for a too general functional dependence. Thus, we restrict later to a causal Volterra-type functional dependence which is also a feature of our inpainting model. Under this causal functional dependence we can prove existence and uniqueness (Theorem 4.14) and also continuous dependence (Theorem 4.16) on the data $c, f$, and $u_{0}$ by a contraction argument. Similar approaches which utilize fixed-point theory for the derivation of local solutions to functional-differential problems posed on the half space can be found in [22] where the author considers classical solutions for time-dependent non-linear PDEs with memory effect.

As a consequence of the general theory, we can positively answer the question of the wellposedness of the inpainting model (1.3): there is a unique solution which depends continuously on the data image $u_{0}$ and the parameters in the model.

1.3. Outline of the Paper. In Section 2 we give a full description of the problem with all its requirements. Section 3 discusses the linear problem in detail: in Section 3.1 we study the characteristic coordinates, in Section 3.2 we prove the existence of a solution, and in Section 3.3 we show that this solution is unique and stable. Section 4 deals with the general quasi-linear problem (1.6) by using fixed-point theory: in Section 4.1 we justify the formulation of the problem as a fixed-point problem, in Section 4.1.1 we prove that a fixed-point exists and in Section 4.1.2 we give an example of non-uniqueness. In Section 4.2 we introduce the concept of causality with respect to the functional argument. Uniqueness and stability of the solution in the case of functional causality are the subjects of Sections 4.2.1 and 4.2.2.

2. The Problem and its Requirements. In this section we will first collect all the requirements and then state the full problem at the end. We begin with the domain.

REQUIREMENT 2.1. Domains $\Omega \subset \mathbb{R}^{2}$ are required to be open, bounded, simply connected, and to have have a $C^{1}$-boundary.

The boundary $\partial \Omega$ is a simple closed $C^{1}$-curve. Throughout this paper we denote by $\gamma: \mathbb{R} \rightarrow$ $\partial \Omega$ a generic periodic parametrization of $\partial \Omega$. Furthermore, by $I=[a, b[\subset \mathbb{R}$ we denote an interval such that $\left.\gamma\right|_{I}$ is a generator of $\gamma$.

In the introduction we talked about the generalized distance-to-boundary function $T$ which induced the pixel serialization in the algorithm. This function is also involved in the continuous model and plays the role of time. Hence, we call $T$ time function. Here we consider time functions whose range corresponds to a finite time interval. That means that these time functions will incorporate a stop set, on which they become maximal. Here we state the geometric properties of admissible stop sets.

REQUIREMENT 2.2. A stop sets $\Sigma$ is a closed subset of $\Omega$, where $\Sigma$ is either an isolated point, or a connected set with tree-like structure. If $\Sigma$ is not an isolated point, we assume that $\Sigma$ is the union of finitely many rectifiable $C^{1}$-arcs $\Sigma_{k}$. The collection $\left\{\Sigma_{k}\right\}_{k=1, \ldots, n}$ is assumed to be minimal in the number $n$ of arcs, so $\Sigma$ is decomposed by breaking it up at corners and branching points. Furthermore, we require for each arc $\Sigma_{k}$ that its relative interior $\Sigma_{k}$ has a given orientation by a continuous unit normal $n_{k}: \Sigma_{k} \rightarrow S^{1}$.

Later on, we will need a concept of one-sided limits towards $z \in \Sigma^{\circ}$.

DEFINITION 2.3. A point $x \in \Omega \backslash \Sigma$ which has a unique closest point $p$ in the interior of some arc $\stackrel{\circ}{\Sigma}_{k}$ is said to be on the plus-side (minus-side) of $\stackrel{\circ}{\Sigma}_{k}$ if

$$
\frac{x-p}{|x-p|}=+n_{k}(p) \quad\left(-n_{k}(p)\right) \text {. }
$$


A sequence $\left(x_{n}\right)_{n \in \mathbb{N}}, x_{n} \in \Omega \backslash \Sigma$ approaches $z \in \Sigma_{k}^{\circ}$ from the plus-side (minus-side) if the sequence converges to $z$ and almost all elements $x_{n}$ are on the plus-side (minus-side) in symbols:

$$
x_{n} \rightarrow z_{+} \quad\left(x_{n} \rightarrow z_{-}\right) .
$$

Next, we collect the properties of admissible time functions. We do this in two steps. In the first collection of requirements we summarize sufficient features which make time functions behave reasonably in between the boundary and the stop set. In the second collection we add requirements considering the behavior close to and on the stop set.

REQUIREMENT 2.4. Time functions $T: \Omega \rightarrow \mathbb{R}$ are defined on a domain $\Omega$ in accordance with Requirement 2.1. and satisfy the following conditions:

1. $T \in C^{2}(\bar{\Omega})$.

2. The boundary of $\Omega$ is the start level: $\left.T\right|_{\partial \Omega}=0$.

3. $T$ incorporates a stop set $\Sigma$ in accordance with Requirement 2.2, i.e. $\Sigma$ is the maximal level of $T$. We norm the maximal value as $\left.T\right|_{\Sigma}=1$.

4. $T$ increases strictly from $\partial \Omega$ to $\Sigma$, i.e., $\nabla T(x)=0 \Leftrightarrow x \in \Sigma$.

5. Good behavior near $\Sigma:$ let $y \in \Sigma, h \in S^{1}$ a unit vector, and let $T(y+r h)=1-\mathcal{O}\left(r^{p}\right)(p=p(y, h))$ as $r \rightarrow 0_{+}$. We require that there is a bound $q$ such that $p(y, h)<q$ for all $y \in \Sigma, h \in S^{1}$.

In the following we denote upper level-sets of $T$ by $\Omega_{T \geq \lambda}:=\{x \in \Omega: T(x) \geq \lambda\}$. Because of Requirement 2.4, the field $N$,

$$
N(x):=\frac{\nabla T(x)}{|\nabla T(x)|}
$$

is well-defined on $\Omega \backslash \Sigma$. $N$ is continuously differentiable and extendable onto $\partial \Omega$ and gives the normals to level lines $\Omega_{T=\lambda}=\partial \Omega_{T \geq \lambda}$. We also need a good behavior of the maps $T$ and $N$ at $\Sigma$.

REQUIREMENT 2.5 .

1. $N$ has one-sided extensions onto the relatively open components $\stackrel{\circ}{\Sigma}_{k}$ and those extensions coincide with $\pm n_{k}$ :

$$
N^{+}(y):=\lim _{x \rightarrow y_{+}} N(x)=-n_{k}(y), \quad N^{-}(y):=\lim _{x \rightarrow y_{-}} N(x)=n_{k}(y)
$$

for every $y \in \stackrel{\circ}{\Sigma}_{k}$.

2. The derivative $D N$ has one-sided extensions onto the relatively open components $\Sigma_{k}^{\circ}$, i.e.,

$$
(D N)^{+}(y):=\lim _{x \rightarrow y_{+}} D N(x), \quad(D N)^{-}(y):=\lim _{x \rightarrow y_{-}} D N(x)
$$

exist for every $y \in \stackrel{\circ}{\Sigma}_{k}$.

3. $|D N| \in L^{1}(\Omega)$, i.e., poles of $|D N|$ at corner-, branching-and terminal nodes of $\Sigma$ are integrable. This feature is assumed to hold in the case that $\Sigma$ is an isolated point as well.

What remains are the assumptions on admissible transport fields and the right-hand sides. Those are as follows.

REQUIREMENT 2.6. Assume that a domain $\Omega$ and a time function $T$ with stop set $\Sigma$ according to the requirements stated above are already specified. Transport fields are maps of the form

$$
c: L^{1}(\Omega) \rightarrow C^{1}(\Omega \backslash \Sigma)^{2}, \text { with } c[.](x): L^{1}(\Omega) \rightarrow \mathbb{R}^{2} \text {, and } c[v]: \Omega \backslash \Sigma \rightarrow \mathbb{R}^{2} \text {. }
$$

and are required to satisfy: ( $D_{x} c[v]$ denotes the derivative of $c[v]$ with respect to $x \in \Omega \backslash \Sigma$.)

1. For fixed $v \in L^{1}(\Omega)$ we have $c[v] \in C^{1}(\Omega \backslash \Sigma)^{2}$ with the properties:

a) $c[v]$ and $D_{x} c[v]$ extend continuously onto $\partial \Omega$.

b) $c[v]$ and $D_{x} c[v]$ have one-sided extensions $\left(c[v]^{+}, c[v]^{-}\right.$respectively $\left.\left(D_{x} c[v]\right)^{+},\left(D_{x} c[v]\right)^{-}\right)$onto the relatively open components $\Sigma_{k}^{\circ}$ of $\Sigma$.

2. Uniform unit speed and inward-pointing condition:

a) $|c[v](x)|=1$ for all $x \in \bar{\Omega} \backslash \Sigma$ and for all $v \in L^{1}(\Omega)$. 
b) There is a uniform lower bound $\beta>0$ such that

$$
\beta \leq\langle c[v](x), N(x)\rangle \leq 1 \quad \forall x \in \bar{\Omega} \backslash \Sigma \quad \text { and } \quad \forall v \in L^{1}(\Omega) .
$$

c) Both conditions a) $\mathcal{E}$ b) hold for the one-sided extensions $c[v]^{+}, c[v]^{-}$of $c[v]$.

3. Uniform bounds and continuity in $v$

a) Let $z_{k}, k \in\{1, \ldots, m\}$ denote the terminal-, branching- and kink nodes of $\Sigma$. For every $\varepsilon>0$, such that each disk $B_{\varepsilon}\left(z_{k}\right)$ is compactly contained in $\Omega$, we set $V_{\varepsilon}:=\Sigma \cup \bigcup_{k=1}^{m} \overline{B_{\varepsilon}\left(z_{k}\right)}$. For every admissible $\varepsilon>0$, there is a bound $M_{\varepsilon}$ such that

$$
\left|D_{x} c[v](x)\right| \leq M_{\mathcal{E}}, \quad \forall x \in \Omega \backslash V_{\mathcal{\varepsilon}}, \quad \text { and } \quad \forall v \in L^{1}(\Omega) .
$$

b) The matrix-valued map $D_{x} c: L^{1}(\Omega) \rightarrow C(\Omega \backslash \Sigma)^{2 \times 2}$ is $L^{1}$-bounded by

$$
\left\|D_{x} c[v]\right\|_{L^{1}(\Omega)}<M_{1} \quad \forall v \in L^{1}(\Omega) .
$$

In particular, for every $v \in L^{1}(\Omega)$, poles of $\left|D_{x} c[v]\right|$ which occur at the terminal-, branching-and kink nodes of $\sum$ are integrable.

c) $c$ is continuous in the following manner: if $v \in L^{1}(\Omega)$ and $\left(v_{n}\right)_{n \in \mathbb{N}}$ is a sequence in $L^{1}(\Omega)$ with $\left\|v-v_{n}\right\|_{L^{1}(\Omega)} \rightarrow 0$, then the sequence of images $c\left[v_{n}\right]$ converges uniformly to $c[v]$,

$$
\left\|c[v]-c\left[v_{n}\right]\right\|_{\infty} \rightarrow 0 .
$$

REQUIREMENT 2.7. Right-hand sides are maps of the form

$$
f: L^{1}(\Omega) \rightarrow C^{1}(\bar{\Omega}), \text { with } f[.](x): L^{1}(\Omega) \rightarrow \mathbb{R}, \text { and } f[v]: \Omega \rightarrow \mathbb{R} .
$$

and are required to satisfy: $\left(\nabla_{x} f[v]\right.$ denotes the derivative of $f[v]$ with respect to $x \in \Omega \backslash \Sigma$.)

a) The map $f$ is bounded by

$$
\|f[v]\|_{\infty} \leq M_{2} \quad \forall v \in L^{1}(\Omega) .
$$

b) The map $\nabla_{x} f: L^{1}(\Omega) \rightarrow C(\bar{\Omega})^{2}$ - the derivative of $f[v]$ w.r.t. the variable $x$ - is bounded by

$$
\left\|\nabla_{x} f[v]\right\|_{\infty} \leq M_{3} \quad \forall v \in L^{1}(\Omega) .
$$

c) $f$ is continuous in the following manner: if $v \in L^{1}(\Omega)$ and $\left(v_{n}\right)_{n \in \mathbb{N}}$ is a sequence in $L^{1}(\Omega)$ with $\left\|v-v_{n}\right\|_{L^{1}(\Omega)} \rightarrow 0$, then the sequence of images $f\left[v_{n}\right]$ converges uniformly to $f[v]$,

$$
\left\|f[v]-f\left[v_{n}\right]\right\|_{\infty} \rightarrow 0 .
$$

Now that we have collected all assumptions, we finally state the problem.

PROBLEM 2.8. We search for $u \in B V(\Omega)$, such that

$$
\begin{aligned}
\langle c[u](x), D u\rangle & =f[u](x) \cdot \mathcal{L}^{2} \quad \text { in } \quad \Omega \backslash \Sigma, \\
\left.u\right|_{\partial \Omega} & =u_{0},
\end{aligned}
$$

where $u_{0} \in B V(\partial \Omega)$ and the coefficients satisfy the requirements above.

Remark: in Section 3.2 we will apply the method of characteristics to construct a solution. Requirement 2.6 part $2 \mathrm{~b}$ ) states that the specified time function $T$ is a global Lyapunov function (cf. [3]) for this dynamical system and the set $\Sigma$ is an attractor. This might seem unnatural, but it is not: considering the Cauchy problem $\partial_{t} u+a \partial_{x} u=0$ on the half-plane $t>0$, we see that the natural time function $T(t, x)=t$ is a Lyapunov function for the flow induced by the transport field $c=(1, a)^{T}$. Note also that the natural time $T(t, x)=t$ is the Euclidean distance to the $x$-axis which is the boundary of the half-plane $t>0$.

Throughout the paper we denote by $\Omega$ a domain, by $\Sigma$ a stop set, by $T$ a time function with the field of normals $N$ and by $c$ a transport field all in accordance with the requirements 2.1, 2.2, $2.4,2.5$, and 2.6. 
3. The Linear Problem. In this section we concentrate on the linear problem

$$
\langle c[v](x), D u\rangle=f[v](x) \cdot \mathcal{L}^{2} \quad \text { in } \Omega \backslash \Sigma,\left.\quad u\right|_{\partial \Omega}=u_{0},
$$

which we obtain by keeping the functional argument fixed with some arbitrary $v \in L^{1}(\Omega)$. Our goal is to construct a global solution $u \in B V(\Omega)$ which is defined on all of $\Omega$. Throughout this section we simplify the notation $c:=c[v], f:=f[v]$ by omitting the functional argument.

3.1. A Customized Coordinate System. Requirements 2.4, 2.5, and 2.6 in combination are such that the family of characteristics gives us a customized coordinate system for the linear problem (3.1). This section reveals the properties of the induced coordinate system. The following lemma yields a bound on the arc-length of characteristics.

LEMMA 3.1.

a) Let $q$ be the bound from Requirement 2.4 part 5 , let $\varphi(t):=-t^{\frac{1}{q}}$ and let

$$
T_{0}(x):=1+\varphi(1-T(x)) .
$$

Then, the gradient $\nabla T_{0}$ of the transformed time function $T_{0}$ blows up at $\Sigma$ and is bounded below $\left|\nabla T_{0}(x)\right| \geq m_{0}>0$, for all $x \in \Omega \backslash \Sigma$, away from zero.

b) For every regular $C^{1}$-curve $x:[0, a[\rightarrow \bar{\Omega} \backslash \Sigma(a=\infty$ admissible $)$ which satisfies the following condition

$$
0<\beta \leq\left\langle N(x(\tau)), \frac{x^{\prime}(\tau)}{\left|x^{\prime}(\tau)\right|}\right\rangle, \quad \forall \tau \in[0, a[,
$$

the arc-length of $x$ is uniformly bounded by

$$
\operatorname{arclength}(x) \leq \frac{1}{\beta \cdot m_{0}}
$$

Proof.

a) The function $T_{0}$ is well-defined since $0 \leq T \leq 1$ and its derivative is $\nabla T_{0}(x)=H(x) \cdot N(x)$ with

$$
H(x):=\frac{1}{q}(1-T(x))^{\frac{1-q}{q}} \cdot|\nabla T(x)|>0 .
$$

Let $y \in \Sigma, h \in S^{1}$ and $r>0$. By Requirement 2.4 part 5 we have $1-T(y+r h)=C_{1} r^{p}$ with $C_{1}>0$. Because $T \in C^{2}(\Omega)$ and $\left.\nabla T\right|_{\Sigma}=0$ we conclude that $|\nabla T(y+r h)|=C_{2} r^{p-1}$ with the same $p$ as before. Consequently, $H$ exhibits the asymptotic

$$
H(y+r h)=C_{3} r^{\frac{p(1-q)}{q}} r^{p-1}=C_{3} r^{\frac{p-q}{q}}
$$

as $r \rightarrow 0_{+}$and, since $q>p(y, h)$ holds uniformly according to Requirement 2.4 part 5, must blow up $H(y+r h) \rightarrow \infty$ for any choice of $y \in \Sigma, h \in S^{1}$.

We will show next that $\left|\nabla T_{0}\right| \geq m_{0}>0$. Assume by contradiction that the infimum of $H=\left|\nabla T_{0}(x)\right|$ is zero. Now, choose an open neighborhood $U$ of $\Sigma$ such that $\left.H\right|_{U} \geq M$ for some constant $M>0$. This is possible because of the blow up. The restriction $\left.H\right|_{\bar{\Omega} \backslash U}$ onto the compact complement $\bar{\Omega} \backslash U$ is a continuous function and must take the minimum at some point $\hat{x} \in \bar{\Omega} \backslash U$, i.e., $H(\hat{x})=0$. But then (3.5) implies $|\nabla T(\hat{x})|=0$ which contradicts Requirement 2.4 part 4 , since $\hat{x} \notin \Sigma$. Thus, $H=\left|\nabla T_{0}\right|$ has a minimum $m_{0}$ greater than zero.

b) Using $T_{0}$ we estimate the arc-length from above by

$$
\begin{aligned}
T_{0}(x(a))-T_{0}(x(0)) & =\int_{0}^{a}\left\langle\nabla T_{0}(x), x^{\prime}\right\rangle d \tau=\int_{0}^{a}\left\langle N(x), \frac{x^{\prime}}{\left|x^{\prime}\right|}\right\rangle \cdot\left|\nabla T_{0}(x)\right| \cdot\left|x^{\prime}\right| d \tau \\
& \geq \beta \int_{0}^{a}\left|\nabla T_{0}(x)\right| \cdot\left|x^{\prime}\right| d \tau \geq \beta \cdot m_{0} \int_{0}^{a}\left|x^{\prime}\right| d \tau=\beta \cdot m_{0} \cdot \operatorname{arclength}(x) .
\end{aligned}
$$


Since $0 \leq T_{0} \leq 1$, we conclude (3.4). The bound on the arc-length depends only on $\beta$ and information from $T$.

Because of its nice properties the transformed version $T_{0}$ defined by (3.2) will be identified with the time variable of the characteristics. Whenever we speak about $T_{0}$ we mean this transformed version of a given time function $T$. The next lemma discusses the globality of the characteristics: they cover all of $\Omega \backslash \Sigma$.

LEMMA 3.2.

a) The characteristic initial value problem (IVP)

$$
y^{\prime}=c(y), \quad y(0)=x \in \Omega \backslash \Sigma,
$$

has a unique maximally continued solution $y:] t_{-}, t_{+}\left[\rightarrow \mathbb{R}^{2}\right.$, with $-\infty<t_{-}<0<t_{+}<\infty$. Every trajectory y connects the sets $\partial \Omega$ and $\Sigma$, i.e.,

$$
\lim _{t \rightarrow t_{-}} y(t) \in \partial \Omega, \quad \quad \quad \lim _{t \rightarrow t_{+}} y(t) \in \Sigma .
$$

For every point $z \in \stackrel{\Sigma}{\Sigma}_{k}$ in the relative interior of some $C^{1}$-arc of $\Sigma$, there are exactly two trajectories which hit $z$ in the limit $t \rightarrow t_{+}$, one for each side of $\Sigma_{k}$.

b) The transformed transport field $c_{0}$

$$
c_{0}:=\frac{c}{\left\langle c, \nabla T_{0}\right\rangle}
$$

extends continuously onto the stop set $\Sigma$ by $\left.c_{0}\right|_{\Sigma} \equiv 0$. Its trajectories $y_{0}$, i.e., solutions of $y^{\prime}=c_{0}(y)$, satisfy

$$
T_{0}\left(y_{0}(\lambda+s)\right)=\lambda+T_{0}\left(y_{0}(s)\right) .
$$

Proof.

a) Because $c$ is Lipschitz continuous there is a maximally continued, unique solution $y$ of (3.6) with time domain $] t_{-}, t_{+}[$and $0 \in] t_{-}, t_{+}[$. Because of the unit speed condition $|c|=1, y$ never stops inside $\Omega \backslash \Sigma$ and never blows up. The inward-pointing condition (2.1) implies, by

$$
\frac{d}{d t} T_{0}(y(t))=\left\langle\nabla T_{0}(y(t)), c(y(t))\right\rangle \geq m_{0} \cdot \beta>0,
$$

that $T_{0}(y(t))$ strictly increases at least by a rate of $m_{0} \cdot \beta$. Thus, $y$ collapses at boundary of $\Omega \backslash \Sigma$. Forward in time, as $t \rightarrow t_{+}, y$ will collapse at $\Sigma$ and backward in time, as $t \rightarrow t_{-}, y$ will collapse at $\partial \Omega$. Because of unit speed the values $t_{+}, t_{-}$mean the arc-length of the piece which connects $x$ with $\Sigma$ and that of the piece which connects $x$ with $\partial \Omega$. Hence, by Lemma $3.1, t_{+}, t_{-}$are finite. Assume now that $z \in \Sigma_{k}^{\circ}$ and consider the side where the normal $n_{k}(z)$ points to. According to Definition $2.3 \mathrm{~b}$ ) we call this side the "plus-side" and the opposite side the "minus-side". Since $c$ and $D c$ both extend from the plus-side onto $\Sigma_{k}^{\circ}$ by $c^{+}$and $(D c)^{+}$, the backward IVP

$$
y^{\prime}=-c(y), \quad y(0)=z, \quad \text { with } c(z):=c^{+}(z) .
$$

has a unique solution that starts at $z \in \stackrel{\Sigma}{\Sigma}_{k}$ and evolves away from $\Sigma$ into the plus-side. Hence, vice versa there is only one solution $y$ of the forward IVP (3.6) that approaches $z$ from the plus-side. The same argumentation holds true for the minus-side.

b) From (3.2) we know that $T_{0} \leq 1$. Consider again the forward IVP (3.6). For $\lambda$ with $T_{0}(x) \leq$ $\lambda<1$, there is a unique time $\tau(\lambda)$, when the trajectory $y$ crosses the $\lambda$-level of $T_{0}$. This is because $T_{0}(y(t))$ increases strictly by (3.8), so $y$ crosses the $\lambda$-level only once . Viewing $\tau$ as a 
function of $\lambda$, the implicit function theorem applied to $T_{0}(y(\tau))=\lambda$ yields the differentiability of $\tau$ with respect to $\lambda$ and the derivative is given by

$$
\tau^{\prime}(\lambda)=\left.\frac{1}{\left\langle\nabla T_{0}(z), c(z)\right\rangle}\right|_{z=y(\tau(\lambda))} .
$$

Using again the inward-pointing condition (2.1) and recalling that $\left|\nabla T_{0}\right| \geq m_{0}>0$, we infer

$$
0<\tau^{\prime} \leq \frac{1}{m_{0} \cdot \beta}
$$

Let $s:=T_{0}(x)$. Then, the function $\tau$ maps $\left[s, 1\left[\right.\right.$ to $\left[0, t_{+}\left[\right.\right.$with $\tau(s)=0$ and $\tau(1)=t_{+}$. Moreover, we have for $\tau^{\prime}(\lambda) \rightarrow 0$ as $\lambda \rightarrow 1$ since $\left|\nabla T_{0}(y(\tau(1)))\right|=\left|\nabla T_{0}\left(y\left(t_{+}\right)\right)\right|=\infty$. Now, we change the independent variable

$$
y_{0}(\lambda):=y(\tau(\lambda)) .
$$

Then, $y_{0}$ satisfies the initial condition $y_{0}(s)=y(0)=x$ and has the derivative

$$
y_{0}^{\prime}(\lambda)=y^{\prime}(\tau(\lambda)) \cdot \tau^{\prime}(\lambda)=\left.\frac{c(z)}{\left\langle\nabla T_{0}(z), c(z)\right\rangle}\right|_{z=y_{0}(\lambda)}=c_{0}\left(y_{0}(\lambda)\right)
$$

Consequently, $y_{0}$ is a trajectory of $c_{0}$ which satisfies, by construction,

$$
T_{0}\left(y_{0}(\lambda+s)\right)=T_{0}(y(\tau(\lambda+s)))=\lambda+s=\lambda+T_{0}(x)=\lambda+T_{0}\left(y_{0}(s)\right) .
$$

Because $\nabla T_{0}$ blows up at $\Sigma$ while $|c|=1$, in the limit we have $c_{0}(y) \rightarrow 0$ as $y \rightarrow z \in \Sigma$ which yields the continuous extension of $c_{0}$ onto $\Sigma$.

Part b) of Lemma 3.2 shows that the time variable of a characteristic $y_{0}$ of the transformed field $c_{0}$ is given by $T_{0}$. We use this feature to introduce new coordinates on $\Omega \backslash \Sigma$ whose conception is similar to polar coordinates on a disk. Whenever we speak about $c_{0}$ we mean the transformed version of a given transport field $c$ according to equation (3.7).

COROLLARY 3.3. Let $\gamma: \mathbb{R} \rightarrow \partial \Omega$, a periodic parametrization of $\partial \Omega$ and let $I=[a, b[\subset \mathbb{R}$ be an interval such that $\gamma(I)=\partial \Omega$. Then, the general solution $\xi(t, s)$ of the forward IVP

$$
y^{\prime}=c_{0}(y), \quad y(0)=\gamma(s),
$$

defines a diffeomorphism $\xi:] 0,1[\times] a, b[\rightarrow \Omega \backslash(\Sigma \cup S)$, where $S:=\{\xi(t, a): t \in] 0,1[\}$. The inverse map $\xi^{-1}(x)=(t(x), s(x))^{T}$ is given by $\xi^{-1}(x)=\left(T_{0}(x), \gamma^{-1}\left(\eta\left(T_{0}(x), x\right)\right)\right)^{T}$ where $\eta(t, x)$ denotes the general solution of the backward IVP

$$
y^{\prime}=-c_{0}(y), \quad y(0)=x \in \Omega \backslash(\Sigma \cup S) .
$$

The relation between $\xi$ and $\eta$ is

$$
\xi(t, s(x))=\eta\left(T_{0}(x)-t, x\right)
$$

Proof. $\xi$ solves the forward IVP and we know from Lemma 3.2 that any curve $\xi(., s)$ is located in $\Omega \backslash \Sigma$ and connects the sets $\partial \Omega$ and $\Sigma$. By this, it is obvious that the set $\Omega \backslash(\Sigma \cup S)$ is simply connected and open in $\mathbb{R}^{2}$, and that $\xi$ maps $] 0,1[\times] a, b[$ onto $\Omega \backslash(\Sigma \cup S)$.

Clearly, $\xi$ is differentiable with respect to time $t$. Since $\gamma \in C^{1}(] a, b[, \partial \Omega)$ and $c_{0} \in C^{1}\left(\Omega \backslash \Sigma, \mathbb{R}^{2}\right)$, we obtain $\partial_{s} \xi$ from the variational equation

$$
\partial_{t}\left(\partial_{s} \xi\right)=D c_{0} \circ \xi \cdot \partial_{s} \xi, \quad \partial_{s} \xi(0, s)=\gamma^{\prime}(s) .
$$


Next we discuss $\eta$, the solution of the backward IVP. From Lemma 3.2 we know the trajectory $\eta(., x)$ hits the boundary after time $t=T_{0}(x)$. Hence, $\eta\left(T_{0}(x), x\right)$ is the unique point on the boundary that corresponds to $x$ which implies further

$$
\eta\left(T_{0}(x), x\right)=\gamma(s(x)) \Rightarrow s(x)=\gamma^{-1}\left(\eta\left(T_{0}(x), x\right)\right) .
$$

Because $\xi(., s(x))$ and $\eta(., x)$ both connect the points $x$ and $\gamma(s(x))$ along the same curve in opposite direction and have the same absolute velocity $\left|c_{0}\right|$, it follows that $t(x)=T_{0}(x)$ and furthermore the relation

$$
\xi(t, s(x))=\eta\left(T_{0}(x)-t, x\right) .
$$

With $t(x)$ and $s(x)$ we have the inverse map $\xi^{-1}(x)=(t(x), s(x))^{T}$. The differentiability properties of $\gamma^{-1}, T_{0}$ and $c_{0}$ imply the differentiability of $\xi^{-1}(x)$ as we obtain $D_{x} \eta$ from the variational equation for the backward IVP

$$
\frac{d}{d t}\left(D_{x} \eta\right)=-D c_{0} \circ \eta \cdot D_{x} \eta, \quad D_{x} \eta(0, x)=I .
$$

In the following lemma, we collect diverse properties of the coordinate system introduced above. We will make use of these properties in the proofs later on where we will often change to characteristic variables.

LEMMA 3.4. Let $\gamma$ and $\xi$ as in Corollary 3.3. Then,

a) if $\gamma$ is clockwise, the Jacobian $D \xi=\left(\partial_{t} \xi \mid \partial_{s} \xi\right)$ of the diffeomorphism $\xi$ has a positive determinant which satisfies the estimate

$$
0<\operatorname{det} D \xi \leq\left|\partial_{t} \xi\right|\left|\partial_{s} \xi\right| \leq \frac{\operatorname{det} D \xi}{\beta}
$$

If $\gamma$ is counter-clockwise, the assertions hold for $-\operatorname{det} D \xi$.

b) for each of the relatively open $C^{1}$-arcs $\Sigma_{k}^{\circ}$ of $\Sigma$, we can find two subsets $J_{k,+}$ and $J_{k,-}$ of I such that the maps $\xi(1, s)$ with $s \in J_{k,+}$ and $\xi(1, s)$ with $s \in J_{k,-}$ are both regular $C^{1}$-parametrizations of $\Sigma_{k}^{\circ}(+$ and - relate to the orientation given by $n_{k}$ ).

c) $D c_{0} \circ \xi \cdot \partial_{s} \xi$ is integrable over $[0,1[\times[a, b[$.

d) The inverse map $\xi^{-1}$ is one-sided extensible onto the relatively open $C^{1}-\operatorname{arcs} \Sigma_{k}^{\circ}$ of $\Sigma$.

Proof.

a) By Lemma 3.2 part b), we have

$$
\left.T_{0}(\xi(t, s))=t, \quad \forall s \in\right] a, b[.
$$

That means that the function $\xi(t,):.] a, b\left[\rightarrow \Omega\right.$ parametrizes the $t$-level of $T_{0}$ and is clockwise if the initial one $\xi(0, s)=\gamma(s)$ was. In this case $\partial_{s} \xi^{\perp}:=\left(-\partial_{s} \xi_{2}, \partial_{s} \xi_{1}\right)$ points to the exterior of $\Omega_{T_{0} \geq t}$, and can be decomposed $\partial_{s} \xi^{\perp}=-N \circ \xi \cdot\left|\partial_{s} \xi\right|$ in terms of the normal field $N$ (which points into the interior) and its magnitude. Since $\partial_{t} \xi$ is aligned with the normed vector $c \circ \xi$, we decompose it similarly $\partial_{t} \xi=c \circ \xi \cdot\left|\partial_{t} \xi\right|$. We conclude that

$$
\pm \operatorname{det} D \xi=\operatorname{det}\left(\partial_{t} \xi \mid \partial_{s} \xi\right)=\left\langle\partial_{t} \xi,-\partial_{s} \xi^{\perp}\right\rangle=\left|\partial_{t} \xi\right|\left|\partial_{s} \xi\right|\langle c, N\rangle \circ \xi
$$

depending on the orientation of $\gamma$. Because $\xi$ is a diffeomorphism, the determinant is never zero. The inward-pointing condition (2.1) implies the positiveness of $\pm \operatorname{det} D \xi$ as well as the relation

$$
\pm \operatorname{det} D \xi \leq\left|\partial_{t} \xi\right|\left|\partial_{s} \xi\right| \leq \pm \frac{\operatorname{det} D \xi}{\beta}
$$



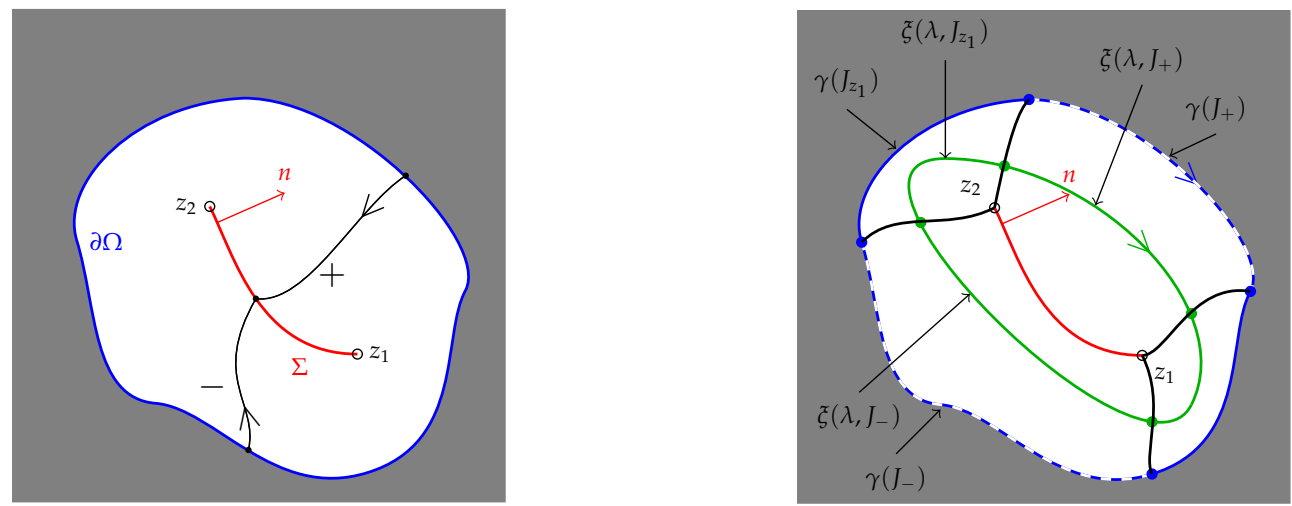

FIG. 3.1. LEFT: The left image shows the domain $\Omega$ in white, the boundary $\partial \Omega$ in blue, and in red the stop set $\Sigma$ with terminal nodes $z_{1}$ and $z_{2}$. The red vector $n$ is the normal of $\Sigma$ and defines the plus and the minus side. The black curves are two characteristics, both start at $\partial \Omega$, but the upper one approaches $\Sigma$ from the plus side while the other comes from the minus side. RIGHT: The right image illustrates the decomposition of the level line $\left\{T_{0}=\lambda\right\}=\xi(\lambda,$.$) , the green curve, into four parts. In black we have the$ characteristics which hit the terminal nodes. For each node only the "first" and "last" one of the characteristics hitting the node are painted. In solid blue we have the parts $\gamma\left(J_{z_{1}}\right)$ and $\gamma\left(J_{z_{2}}\right)$ which comprise all the start points for which characteristics hit terminal nodes of $\Sigma$. The complement is painted dashed blue and consists of the two parts $\gamma\left(J_{+}\right)$and $\gamma\left(J_{-}\right)$. Here, $\gamma\left(J_{+}\right)\left(\gamma\left(J_{-}\right)\right)$contains all start points for which characteristics approach $\Sigma$ from the plus side (minus side) and end in the relative interior of $\Sigma$. With parameter sets $J_{z_{1}}, J_{z_{2}}, J_{+}$, and $J_{-}$, that correspond to the decomposition of the boundary curve $\gamma$, we obtain an analogous decomposition of the level line $\left\{T_{0}=\lambda\right\}=\xi(\lambda,$.$) by \xi\left(\lambda, J_{z_{1}}\right), \xi\left(\lambda, J_{z_{2}}\right), \xi\left(\lambda, J_{+}\right), \xi\left(\lambda, J_{-}\right)$. If now $\lambda \rightarrow 1$, i.e., $\left\{T_{0}=\lambda\right\} \rightarrow \Sigma$, the curves $\xi\left(1, J_{+}\right)$ and $\xi\left(1, J_{-}\right)$remain and give two parametrizations of $\Sigma$.

b) From the proof of Lemma 3.2 part b) we know that $\xi$ by $\xi(\lambda, s)=y(\tau(\lambda, s)$,s) is related to the original characteristic problem $y^{\prime}=c(y)$ with initial value $y(0, s)=\gamma(s)$, where $\tau$ was implicitly defined by

$$
T_{0}(y(\tau, s))=\lambda, \quad 0 \leq \lambda<1 .
$$

Hence, $\tau$ depends on $s \in\left[a, b\left[\right.\right.$ as well. Clearly, $\tau$ is periodic in $s$ and $C^{1}$-smooth on $[0,1[\times$ $\left[a, b\left[\right.\right.$. The question here is what happens in the limit $\lambda \rightarrow 1$. Since $T_{0}(\xi(1, s))=1$ implies $\xi(1, s) \in \Sigma$, we see that $s \rightarrow \xi(1, s)$ parametrizes $\Sigma$. From Lemma 3.2 part a) we know that if $z \in \Sigma_{k}$ then there are only two trajectories which meet at $z$, one for each side of $\Sigma_{k}$. Now, let the set $J$ consist of all $s \in] a, b\left[\right.$ such that $\xi(1, s)$ is a terminal node of one of the arcs $\Sigma_{1}, \ldots, \Sigma_{n}$. In the set $J_{k,+}$ we collect all elements of $I \backslash J$ such that the family of curves

$$
\xi(\lambda, .): J_{k,+} \rightarrow \Omega_{T_{0}=\lambda}, \quad s \rightarrow \xi(\lambda, s)
$$

reaches the $\operatorname{arc} \Sigma_{k}^{\circ}$ from the plus-side in the limit $\lambda \rightarrow 1$. Analogously, considering the minusside, we choose $J_{k,-}$. For an illustration see Figure 3.1 in which $\Sigma=\Sigma_{1}$ is oriented by $n_{1}$ and $\gamma$ is clockwise. For $s \in J_{k,+}$ we extend the right-hand side of the original IVP by $c^{+}$, then both limits

$$
\lim _{t \rightarrow t_{+}(s)} y(t, s)=z(s) \in \stackrel{\Sigma}{\Sigma}_{k}, \quad \quad \lim _{t \rightarrow t_{+}(s)} y^{\prime}(t, s)=c^{+}(z(s)),
$$

exist and the equation $T_{0}(y(\tau, s))=1$ has the unique solution $\tau(1, s)=t_{+}(s)$. Since $\left|\nabla T_{0}\right|$ blows up at $\Sigma$ (see Lemma 3.1 part a)), we are not finished with the properties of $\partial_{s} \tau$. The continuity of $\tau(1, s)=t_{+}(s)$ is a consequence of the mean value theorem by

$$
\begin{aligned}
|\tau(1, s)-\tau(1, p)| & \leq|\tau(1, s)-\tau(\lambda, s)|+|\tau(\lambda, s)-\tau(\lambda, p)|+|\tau(\lambda, p)-\tau(1, p)| \\
& \leq\left|\partial_{\lambda} \tau\left(\lambda_{1}, s\right)\right||1-\lambda|+|\tau(\lambda, s)-\tau(\lambda, p)|+\left|\partial_{\lambda} \tau\left(\lambda_{2}, p\right)\right||1-\lambda| \\
& \leq \frac{2}{m_{0} \cdot \beta}|1-\lambda|+|\tau(\lambda, s)-\tau(\lambda, p)| .
\end{aligned}
$$


Similarly, we obtain the continuous extension of $\tau(\lambda, s)$ by $\tau(1, s)$ :

$$
|\tau(1, s)-\tau(\lambda, p)| \leq \frac{1}{m_{0} \cdot \beta}|1-\lambda|+|\tau(\lambda, s)-\tau(\lambda, p)| .
$$

As a first result we have that the parametrization $\xi(1, s)=y(\tau(1, s), s)$ of the arc $\Sigma_{k}^{\circ}$ is continuous. Now, we study the partial derivative $\partial_{s} \tau$. The implicit function theorem applied to (3.11) gives

$$
0=\left\langle\nabla T_{0}, \partial_{t} y\right\rangle \partial_{s} \tau+\left\langle\nabla T_{0}, \partial_{s} y\right\rangle \quad \Rightarrow \quad \partial_{s} \tau=-\frac{\left\langle N, \partial_{s} y\right\rangle}{\langle N, c\rangle},
$$

Because $c$ and $N$ have continuous one-sided extensions, we have

$$
\lim _{\lambda \rightarrow 1} N(y(\tau(\lambda, s), s))=N^{+}(z(s)), \quad \quad \lim _{\lambda \rightarrow 1} c(y(\tau(\lambda, s), s))=c^{+}(z(s))
$$

and thus, it suffices to look at $\partial_{s} y$. The IVP for $\partial_{s} y$ gives us

$$
\partial_{s} y(t, s)=\gamma^{\prime}(s)+\int_{0}^{t} D c \circ y(h, s) \cdot \partial_{s} y(h, s) d h .
$$

By Requirement 2.6 part 1a) $c$ and $D c$ both extend continuously, and by Requirement 2.6 part 3a) $|D c \circ y(h, s)|$ has a uniform bound when we restrict the domain of $s$ to a small $\varepsilon$ neighborhood $U_{\mathcal{\varepsilon}}(\sigma)$ of say $\sigma \in J_{k,+}$

$$
|D c \circ y(h, s)| \leq M, \quad(h, s) \in[0, \tau(1, s)] \times U_{\mathcal{\varepsilon}}(\sigma) .
$$

Gronwall's lemma (cf. [32]) yields the bound

$$
\left|\partial_{s} y(t, s)\right| \leq\left|\gamma^{\prime}(s)\right| e^{M t} \leq\left|\gamma^{\prime}(s)\right| e^{M \tau(1, s)},
$$

which implies the existence of $\lim _{\lambda \rightarrow 1} \partial_{s} y(\tau(\lambda, s), s)$. The continuity of this limit as function of $s$ follows from the mean value theorem, the established bounds, and the corresponding continuity of $\tau$ :

$$
\left|\partial_{s} y(\tau(1, s), s)-\partial_{s} y(\tau(\lambda, s), s)\right| \leq M\left|\gamma^{\prime}(s)\right| e^{M \tau(1, s)}|\tau(1, s)-\tau(\lambda, s)| .
$$

The continuous extension of $(\lambda, p) \rightarrow \partial_{s} y(\tau(\lambda, p), p)$ follows from this and the triangle inequality. From (3.12) we know that $\partial_{s} \tau(\lambda, s)$ has the same continuity properties as $\partial_{s} y$. Summarizing, we have for $s \in J_{k,+}$ that

$$
\partial_{s} \xi(\lambda, s)=\partial_{t} y(\tau(\lambda, s), s) \cdot \partial_{s} \tau(\lambda, s)+\partial_{s} y(\tau(\lambda, s), s)
$$

extends continuously onto $\lambda=1$ by $\partial_{s} \xi(1, s)$. Finally, for the regularity of $\partial_{s} \xi(1, s)$, we have to show that $\left|\partial_{s} \xi(1, s)\right| \neq 0$. In part a), we have already used that $\partial_{s} \xi=\left|\partial_{s} \xi\right| \cdot N^{\perp}$ and that

$$
\operatorname{det}\left(c \mid \partial_{s} \xi\right)=\left|\partial_{s} \xi\right| \operatorname{det}\left(c \mid N^{\perp}\right)=\left|\partial_{s} \xi\right|\langle c, N\rangle \geq\left|\partial_{s} \xi\right| \cdot \beta .
$$

Now, we substitute (3.13) into the left-hand side of (3.14). Bearing in mind that $\partial_{t} y=c \circ y$, we obtain

$$
\left|\partial_{s} \xi(\lambda, s)\right|\langle c, N\rangle=\operatorname{det}\left(c \circ y(\tau(\lambda, s), s) \mid \partial_{s} y(\tau(\lambda, s), s)\right) .
$$

Hence, $\left|\partial_{s} \xi(\lambda, s)\right|$ becomes zero if and only if $\operatorname{det}\left(c \circ y \mid \partial_{s} y\right)$ does. It is easy to check, that the determinant $\operatorname{det}\left(c \circ y \mid \partial_{s} y\right)$ satisfies the IVP

$\frac{d}{d t} \operatorname{det}\left(c \circ y \mid \partial_{s} y\right)=\operatorname{trace}(D c \circ y) \cdot \operatorname{det}\left(c \circ y \mid \partial_{s} y\right),\left.\quad \operatorname{det}\left(c \circ y \mid \partial_{s} y\right)\right|_{t=0}=\operatorname{det}\left(c \circ \gamma(s) \mid \gamma^{\prime}(s)\right)>0$ 
Thus, the determinant has the same sign as the initial value and being an exponential function cannot become zero within finite time:

$$
\lim _{t \rightarrow t_{+}(s)} \operatorname{det}\left(c \circ y(t, s) \mid \partial_{s} y(t, s)\right) \neq 0 .
$$

Hence, we have $\left|\partial_{s} \xi(1, s)\right| \neq 0$ and the parametrization is regular.

c) By the chain rule, the derivative of $c_{0}=c /\left\langle c, \nabla T_{0}\right\rangle$ is

$$
D c_{0}=\frac{1}{\left\langle c, \nabla T_{0}\right\rangle}\left(D c-\frac{c}{\left\langle c, \nabla T_{0}\right\rangle}\left(\nabla T_{0}^{T} \cdot D c+c^{T} \cdot D^{2} T_{0}\right)\right) .
$$

Using that $\partial_{s} \xi= \pm N^{\perp} \circ \xi \cdot\left|\partial_{s} \xi\right|$ and $\left|\partial_{t} \xi\right|=1 /\left\langle c, \nabla T_{0}\right\rangle$, we write the product $D c_{0} \circ \xi \cdot \partial_{s} \xi$ as

$$
D c_{0} \circ \xi \cdot \partial_{s} \xi= \pm\left|\partial_{t} \xi\right|\left|\partial_{s} \xi\right| \cdot\left(D c \cdot N^{\perp}-\frac{c}{\langle c, N\rangle}\left(N^{T} \cdot D c \cdot N^{\perp}+c^{T} \cdot \frac{D^{2} T_{0}}{\left|\nabla T_{0}\right|} \cdot N^{\perp}\right)\right) \circ \xi .
$$

Inspecting the derivative of $N=\nabla T_{0} /\left|\nabla T_{0}\right|$ we see that

$$
D N=\left(I-N \cdot N^{T}\right) \cdot \frac{D^{2} T_{0}}{\left|\nabla T_{0}\right|}=N^{\perp} \cdot N^{\perp T} \cdot \frac{D^{2} T_{0}}{\left|\nabla T_{0}\right|} \Rightarrow c^{T} \cdot \frac{D^{2} T_{0}}{\left|\nabla T_{0}\right|} \cdot N^{\perp}=N^{\perp T} \cdot D N \cdot c .
$$

Finally, we estimate

$$
\begin{aligned}
\left|D c_{0} \circ \xi \cdot \partial_{s} \xi\right| & \leq\left|\partial_{t} \xi\right|\left|\partial_{s} \xi\right|\left(\left|D c \cdot N^{\perp}\right|+\frac{|c|}{\langle c, N\rangle}\left(\left|N^{T} \cdot D c \cdot N^{\perp}\right|+\left|N^{\perp T} \cdot D N \cdot c\right|\right)\right) \circ \xi \\
& \leq \frac{\operatorname{det} D \xi}{\beta}\left(|D c|+\frac{1}{\beta}(|D c|+|D N|)\right) \circ \xi
\end{aligned}
$$

where we have used (3.10) in the last step. The right-hand side of the last inequality is integrable over $] 0,1[\times[a, b[$. This follows from resubstituting $\xi$ and the integrability requirements on $D c$ and $D N$ over $\Omega$ (cf. Requirements 2.6 and 2.5).

d) Let $z \in \Sigma_{k}^{\circ}$. For the plus-side there is a unique $s \in J_{k,+}$ with $\xi(1, s)=z$. By this we define $s^{+}(z)$, which extends the $s(x)$ component of $\xi^{-1}(x)$. Since $t(x)=T_{0}(x)$ is defined on $\Sigma$, we are done.

Remark: A side effect of Lemma 3.4 part c) is that

$$
\partial_{s} \xi(1, s)=\gamma^{\prime}(s)+\int_{0}^{1} D c_{0} \circ \xi(\tau, s) \cdot \partial_{s} \xi(\tau, s) d \tau, \quad s \in J_{k,+}
$$

is integrable over $J_{k,+}$, and dominated convergence yields the rule

$$
\lim _{t \rightarrow 1} \int_{J_{k,+}} \partial_{s} \xi(t, s) d s=\int_{J_{k,+}} \partial_{s} \xi(1, s) d s .
$$

3.2. Existence of a Solution. In this section we prove the existence of a solution to the linear problem (3.1) in the space of functions of bounded variation. We construct a candidate solution with the method of characteristics. First we scale the PDE of (3.1) with the positive factor $1 /\left\langle c(x), \nabla T_{0}(x)\right\rangle$ to get the equivalent PDE

$$
\left\langle c_{0}(x), D u\right\rangle=f_{0}(x) \cdot \mathcal{L}^{2} \quad \text { in } \quad \Omega \backslash \Sigma,
$$

with

$$
f_{0}(x)=\frac{f(x)}{\left\langle c(x), \nabla T_{0}(x)\right\rangle} \quad \text { and } \quad c_{0}(x)=\frac{c(x)}{\left\langle c(x), \nabla T_{0}(x)\right\rangle}
$$


The latter is the transformed transport field as in (3.7). For PDE (3.16) the characteristic equation is exactly the forward IVP from Corollary 3.3 and its solution $\xi$ gives the family of forward characteristics. Similarly, the solution $\eta$ of the backward IVP from Corollary 3.3 gives the family of backward characteristics. Let now $v(t, s):=u \circ \xi(t, s)$. Then, at least formally, the partial derivative of $v$ with respect to $t$ is given by

$$
\partial_{t} v(t, s)=\left\langle\nabla u \circ \xi(t, s), \partial_{t} \xi(t, s)\right\rangle=\left\langle\nabla u, c_{0}\right\rangle \circ \xi(t, s)=f_{0} \circ \xi(t, s) .
$$

Apart from that $v$ satisfies the initial condition $\left(\gamma^{*}\right.$ denotes the pull-back operation)

$$
v(0, s)=u(\xi(0, s))=u(\gamma(s))=u_{0}(\gamma(s))=\gamma^{*} u_{0}(s) .
$$

Thus, by the fundamental theorem of calculus, we obtain

$$
v(t, s)=\gamma^{*} u_{0}(s)+\int_{0}^{t} f_{0} \circ \xi(\tau, s) d \tau .
$$

The function $v$ represents our candidate solution $u$ in characteristic variables $(t, s)$. By using the inverse map $\xi^{-1}$ from Corollary 3.3 and the relation (3.9) between $\xi$ and $\eta$, we push $v$ forward onto $\Omega \backslash \Sigma$ to have $u=v \circ \xi^{-1}$ in original variables $x$

$$
u(x)=u_{0}\left(\eta\left(T_{0}(x), x\right)\right)+\int_{0}^{T_{0}(x)} f_{0} \circ \eta(\tau, x) d \tau .
$$

For the analysis of the candidate solution, it is useful to decompose it additively as

$$
\begin{array}{ll}
v_{1}(t, s)=\gamma^{*} u_{0}(s) \quad, \quad v_{2}(t, s)=\int_{0}^{t} f_{0} \circ \xi(\tau, s) d \tau, \\
u_{1}(x)=u_{0}\left(\eta\left(T_{0}(x), x\right)\right), \quad u_{2}(x)=\int_{0}^{T_{0}(x)} f_{0} \circ \eta(\tau, x) d \tau .
\end{array}
$$

The lemma below shows that the so-constructed candidate belongs to $B V$, the space of functions which the linear problem (3.1) as well as the quasi-linear Problem 2.8 is stated for.

LEMMA 3.5. The candidate solution $u$ from (3.18) with its decomposition $u=u_{1}+u_{2}$ from (3.19) has the properties:

a) $u$ is an element of $B V(\Omega \backslash \Sigma) \cap L^{\infty}(\Omega)$. Its derivative measure is

$$
D u=c_{0}^{\perp}(x) \cdot \mu+\nabla u_{2}(x) \cdot \mathcal{L}^{2} \text { with } \mu:=\xi_{\sharp}\left(\mathcal{L}^{1} \otimes D \gamma^{*} u_{0}\right),
$$

and the total variation is bounded by $|D u|(\Omega \backslash \Sigma) \leq M_{\Omega \backslash \Sigma}$ with

$$
M_{\Omega \backslash \Sigma}:=\frac{\left|D u_{0}\right|}{\beta \cdot m_{0}}+\left(\frac{\|f\|_{\infty}}{\beta}+\frac{\|\nabla f\|_{\infty}}{\beta^{2} \cdot m_{0}}\right) \cdot \mathcal{L}^{2}(\Omega)+\frac{\|f\|_{\infty}}{\beta^{3} \cdot m_{0}^{2}} \cdot\left(\|D c\|_{L^{1}(\Omega)}+\|D N\|_{L^{1}(\Omega)}\right) .
$$

The $L^{\infty}(\Omega)$-norm is bounded by

$$
\|u\|_{L^{\infty}(\Omega)} \leq\left\|u_{0}\right\|_{L^{\infty}(\partial \Omega)}+\frac{\|f\|_{\infty}}{\beta \cdot m_{0}} .
$$


b) $u$ extends onto $\Sigma$, i.e., $u$ is an element of $B V(\Omega) \cap L^{\infty}(\Omega)$. Compared to part a) the extension introduces in the derivative $D u$ an additional jump part for every $C^{1}-\operatorname{arc} \Sigma_{k}$ of $\Sigma$ :

$$
D u=c_{0}^{\perp}(x) \cdot \mu+\nabla u_{2}(x) \cdot \mathcal{L}^{2}+\sum_{k=1}^{n}\left(u_{\Sigma_{k}}^{+}(x)-u_{\Sigma_{k}}^{-}(x)\right) n_{k}(x) \cdot \mathcal{H}^{1}\left\llcorner\Sigma_{k},\right.
$$

where $u_{\Sigma_{k}}^{-}$and $u_{\Sigma_{k}}^{+}$are the left and right interior $B V$-traces of $u$ on $\Sigma_{k}$. The bound on the total variation is added up by

$$
|D u|(\Omega) \leq M_{\Omega \backslash \Sigma}+2 \cdot\|u\|_{L^{\infty}(\Omega)} \cdot \mathcal{H}^{1}(\Sigma) .
$$

Before going into the details of the proof, we summarize some facts concerning the change of variables. If $\varphi \in C_{c}^{1}(\Omega)$ or $\varphi \in C_{c}^{1}(\Omega \backslash \Sigma)$ is a test function, we will denote by $\psi(t, s):=\varphi$ 。 $\xi(t, s)$ the test function in characteristic variables. By the chain rule we then obtain the derivative $\nabla_{t, s} \psi=D \xi^{T} \cdot \nabla_{x} \varphi \circ \xi$ with respect to $(t, s)$. By inversion we get

$$
\operatorname{det} D \xi \cdot \nabla_{x} \varphi \circ \xi=\left(-\partial_{s} \xi^{\perp} \mid \partial_{t} \xi^{\perp}\right) \cdot \nabla_{t, s} \psi .
$$

Let $l=l(k)$ be the non-trivial permutation of $\{1,2\}$, then we write for the $k$-th component

$$
\operatorname{det} D \xi \cdot \partial_{k} \varphi \circ \xi=(-1)^{l} \cdot\left(\partial_{s} \xi_{l} \partial_{t} \psi-\partial_{t} \xi_{l} \partial_{s} \psi\right)=(-1)^{l} \cdot\left(\partial_{t}\left(\partial_{s} \xi_{l} \cdot \psi\right)-\partial_{s}\left(\partial_{t} \xi_{l} \cdot \psi\right)\right) \text {. }
$$

The last equality can easily be derived from the product rule. Finally, we remark that $\psi(t, s)$ is periodic in the variable $s$, since $\xi$ is, i.e., $\psi(t, a)=\psi(t, b)$.

Proof. (of Lemma 3.5) First, we compute the derivative measure $D u$, which in both parts is the same process. Let $\varphi \in C_{c}^{1}(\Omega)$ be a test function. For the moment we restrict the discussion to lower level-sets of $T_{0}$ denoted by $\Omega_{T_{0} \leq \lambda}$ for $0<\lambda<1$. Note that $\Omega_{T_{0} \leq 1}=\Omega$.

When later on we have $\varphi \in C_{c}^{1}(\Omega \backslash \Sigma)$ (respectively $\varphi \in C_{c}^{1}(\Omega \backslash \Sigma)^{2}$ ), as is the case for part a), we will choose $\lambda$ big enough such that $\operatorname{supp} \varphi \subset \Omega_{T_{0} \leq \lambda}$. For part b) we will pass to the limit $\lambda \rightarrow 1$ instead.

The derivatives of $u_{1}$ and $u_{2}$ from the decomposition (3.19) will be computed separately. For $D_{k} u_{1}$ we have to study the following integral:

$$
\begin{aligned}
\int_{\Omega_{T_{0} \leq \lambda}} u_{1}(x) \partial_{k} \varphi(x) d x & =\int_{a}^{b} \int_{0}^{\lambda} v_{1}(t, s) \partial_{k} \varphi \circ \xi(t, s) \operatorname{det} D \xi(t, s) d t d s \\
& =(-1)^{l} \int_{a}^{b} \int_{0}^{\lambda} \gamma^{*} u_{0}(s)\left(\partial_{t}\left(\partial_{s} \xi_{l} \cdot \psi\right)-\partial_{s}\left(\partial_{t} \xi \cdot \psi\right)\right) d t d s .
\end{aligned}
$$

By changing the order of integration, using the integration-by-parts formula for functions of one variable, and the fact that $\gamma^{*} u_{0}$ is a periodic $B V$-function, one obtains

$$
\begin{aligned}
& =(-1)^{l} \int_{a}^{b} \gamma^{*} u_{0}(s) \int_{0}^{\lambda} \partial_{t}\left(\partial_{s} \xi_{l} \cdot \psi\right) d t d s-(-1)^{l} \int_{0}^{\lambda} \int_{a}^{b} \gamma^{*} u_{0}(s) \partial_{s}\left(\partial_{t} \xi_{l} \cdot \psi\right) d s d t \\
& =(-1)^{l} \int_{a}^{b} \gamma^{*} u_{0}(s)\left[\partial_{s} \xi_{l} \cdot \psi\right]_{t=0}^{\lambda} d s+(-1)^{l} \int_{0}^{\lambda} \int_{a}^{b} \partial_{t} \xi_{l} \cdot \psi d D \gamma^{*} u_{0}(s) d t .
\end{aligned}
$$

Because $\varphi$ has compact support in $\Omega$, we have furthermore $\psi(0, s)=\varphi \circ \xi(0, s)=\varphi(\gamma(s))=0$, so the result reduces to

$$
=(-1)^{l} \int_{a}^{b} v_{1}(\lambda, s) \partial_{s} \xi_{l}(\lambda, s) \cdot \psi(\lambda, s) d s+(-1)^{l} \int_{a}^{b} \int_{0}^{\lambda} \partial_{t} \xi_{l} \cdot \psi d t d D \gamma^{*} u_{0}(s) .
$$


For the vector-valued version we test with $\varphi \in C_{\mathcal{c}}^{1}(\Omega)^{2}$ and obtain

$$
\begin{gathered}
\int_{\Omega_{T_{0} \leq \lambda}} u_{1}(x) \operatorname{div} \varphi(x) d x=\int_{\Omega_{T_{0} \leq \lambda}} u_{1}(x) \partial_{1} \varphi_{1}(x) d x+\int_{\Omega_{T_{0} \leq \lambda}} u_{1}(x) \partial_{2} \varphi_{2}(x) d x \\
=-\int_{a}^{b}\left\langle\psi(\lambda, s), \partial_{s} \xi^{\perp}(\lambda, s)\right\rangle v_{1}(\lambda, s) d s-\int_{a}^{b} \int_{0}^{\lambda}\left\langle\psi, \partial_{t} \xi^{\perp}\right\rangle d t d D \gamma^{*} u_{0}(s) .
\end{gathered}
$$

By once more using the relations $\partial_{t} \xi^{\perp}=c_{0}^{\perp} \circ \xi, \partial_{s} \xi^{\perp}=-N \circ \xi \cdot\left|\partial_{s} \xi\right|$, the last result can be written as

$\int_{\Omega_{T_{0} \leq \lambda}} u_{1}(x) \operatorname{div} \varphi(x) d x=\int_{a}^{b}\left(\langle\varphi, N\rangle \cdot u_{1}\right) \circ \xi(\lambda, s)\left|\partial_{s} \xi(\lambda, s)\right| d s-\int_{a}^{b} \int_{0}^{\lambda}\left\langle\varphi, c_{0}^{\perp}\right\rangle \circ \xi d t d D \gamma^{*} u_{0}(s)$.

The first summand integrates with respect to the one-dimensional Hausdorff measure $\mathcal{H}^{1}$ along the $\lambda$-level of $T_{0}$. For the restriction of $\mathcal{H}^{1}$ onto $\lambda$-levels of $T_{0}$ we will use the notation $\mathcal{H}^{1}\llcorner\lambda:=$ $\mathcal{H}^{1}\left\llcorner\Omega_{T_{0}=\lambda}\right.$. In the second integral we change variables by pushing-forward the product measure $\mathcal{L}^{1} \otimes D \gamma^{*} u_{0}$ with the diffeomorphism $\xi$. Let $\mu$ denote the pushed-forward measure

$$
\mu:=\xi_{\sharp}\left(\mathcal{L}^{1} \otimes D \gamma^{*} u_{0}\right),
$$

then we finally obtain

$$
\int_{\Omega_{T_{0} \leq \lambda}} u_{1}(x) \operatorname{div} \varphi(x) d x=\int_{\Omega}\langle\varphi(x), N(x)\rangle u_{1}(x) d \mathcal{H}^{1}\left\llcorner\lambda(x)-\int_{\Omega_{T_{0} \leq \lambda}}\left\langle\varphi(x), c_{0}^{\perp}(x)\right\rangle d \mu(x) .\right.
$$

For the derivative of $u_{2}$ we perform the same steps as above with the integral

$$
\int_{\Omega_{T_{0} \leq \lambda}} u_{2}(x) \partial_{k} \varphi(x) d x=(-1)^{l} \int_{a}^{b} \int_{0}^{\lambda} v_{2}(t, s)\left(\partial_{t}\left(\partial_{s} \xi_{l} \cdot \psi\right)-\partial_{s}\left(\partial_{t} \xi_{l} \cdot \psi\right)\right) d t d s .
$$

We change the order of integration, perform integration by parts, and use the periodicity in $s$ to arrive at

$$
=(-1)^{l} \int_{a}^{b} v_{2}(\lambda, s) \partial_{s} \xi_{l}(\lambda, s) \cdot \psi(\lambda, s) d s-\int_{a}^{b} \int_{0}^{\lambda}(-1)^{l}\left(\partial_{s} \xi_{l} \partial_{t} v_{2}-\partial_{t} \xi_{l} \partial_{s} v_{2}\right) \cdot \psi d t d s .
$$

Because of $v_{2}=u_{2} \circ \xi$ we can, according to (3.22), substitute the last integrand to get

$$
=(-1)^{l} \int_{a}^{b} v_{2}(\lambda, s) \partial_{s} \xi_{l}(\lambda, s) \cdot \psi(\lambda, s) d s-\int_{a}^{b} \int_{0}^{\lambda} \operatorname{det} D \xi \cdot \partial_{k} u_{2} \circ \xi \cdot \psi d t d s .
$$

By means of the last result and a change of variables, we end up with

$$
\int_{\Omega_{T_{0} \leq \lambda}} u_{2}(x) \operatorname{div} \varphi(x) d x=\int_{\Omega}\langle\varphi(x), N(x)\rangle u_{2}(x) d \mathcal{H}^{1}\left\llcorner\lambda(x)-\int_{\Omega_{T_{0} \leq \lambda}}\left\langle\varphi(x), \nabla u_{2}(x)\right\rangle d x,\right.
$$

when testing with $\varphi \in C_{c}^{1}(\Omega)^{2}$. Adding the partial results for $u_{1}$ and $u_{2}$ gives us

$$
\begin{aligned}
\int_{\Omega_{T_{0}} \leq \lambda} u(x) \operatorname{div} \varphi(x) d x & =\int_{\Omega}\langle\varphi(x), N(x)\rangle u(x) d \mathcal{H}^{1}\llcorner\lambda(x) \\
& -\int_{\Omega_{T_{0} \leq \lambda}}\left\langle\varphi(x), c_{0}^{\perp}(x)\right\rangle d \mu(x)-\int_{\Omega_{T_{0} \leq \lambda}}\left\langle\varphi(x), \nabla u_{2}(x)\right\rangle d x .
\end{aligned}
$$

Now, we are ready to turn to the proof of the assertions a) and b). 
a) In this part we have $\Omega \backslash \Sigma$ as the domain of $u$. If we test with $\varphi \in C_{\mathcal{c}}^{1}(\Omega \backslash \Sigma)^{2}$, we can choose $\lambda<1$ so big that (3.23) reduces to

$$
\int_{\Omega} u(x) \operatorname{div} \varphi(x) d x=-\int_{\Omega}\left\langle\varphi(x), c_{0}^{\perp}(x) d \mu(x)+\nabla u_{2}(x) d x\right\rangle .
$$

Thus, in this case the derivative measure is given by

$$
D u=c_{0}^{\perp}(x) \cdot \mu+\nabla u_{2}(x) \cdot \mathcal{L}^{2} .
$$

What remains to show is the boundedness of $\|u\|_{B V(\Omega \backslash \Sigma)}$.

For the total variation $|D u|(\Omega \backslash \Sigma)$ we estimate both summands separately, beginning with

$$
\begin{aligned}
\left|\int_{\Omega}\left\langle\varphi, c_{0}^{\perp}\right\rangle d \mu(x)\right| & =\left|\int_{a}^{b} \int_{0}^{1}\left\langle\varphi, c_{0}^{\perp}\right\rangle \circ \xi d t d D \gamma^{*} u_{0}(s)\right| \leq \int_{a}^{b} \int_{0}^{1}\left|c_{0} \circ \xi\right| d t d\left|D \gamma^{*} u_{0}\right|(s) \cdot\|\varphi\|_{\infty} \\
& \leq \frac{1}{\beta \cdot m_{0}} \cdot \int_{a}^{b} d\left|D \gamma^{*} u_{0}\right|(s) \cdot\|\varphi\|_{\infty}=\frac{\left|D u_{0}\right|}{\beta \cdot m_{0}} \cdot\|\varphi\|_{\infty} .
\end{aligned}
$$

Clearly, the total variation of this summand is bounded by

$$
\left|c_{0}^{\perp}(x) \cdot \mu\right|(\Omega \backslash \Sigma) \leq \frac{\left|D u_{0}\right|}{\beta \cdot m_{0}},
$$

which is the total variation of the boundary data times the bound on the arc-lengths of characteristics from Lemma 3.1. The total variation of the second summand is exactly the $L^{1}$-norm of $\nabla u_{2}$ :

$$
\begin{aligned}
\int_{\Omega}\left|\nabla u_{2}(x)\right| d x & =\int_{a}^{b} \int_{0}^{1}\left|\nabla u_{2} \circ \xi \cdot \operatorname{det} D \xi\right| d t d s=\int_{a}^{b} \int_{0}^{1}\left|-\partial_{s} \xi^{\perp} \partial_{t} v_{2}+\partial_{t} \xi^{\perp} \partial_{s} v_{2}\right| d t d s \\
& \leq \int_{a}^{b} \int_{0}^{1}\left|\partial_{s} \xi\right|\left|\partial_{t} v_{2}\right| d t d s+\int_{a}^{b} \int_{0}^{1}\left|\partial_{t} \xi\right|\left|\partial_{s} v_{2}\right| d t d s
\end{aligned}
$$

This step is completed if the last two integrals are bounded. We write $\partial_{t} v_{2}=f_{0} \circ \xi$ and use (3.10) to get

$$
\left|\partial_{s} \xi\right|\left|\partial_{t} v_{2}\right|=\left|\partial_{s} \xi\right|\left|f_{0} \circ \xi\right|=\left|\partial_{s} \xi\right|\left|\frac{f}{\left\langle c, \nabla T_{0}\right\rangle} \circ \xi\right|=\left|\partial_{s} \xi\right|\left|\partial_{t} \xi\right||f \circ \xi| \leq \frac{\operatorname{det} D \xi}{\beta}|f \circ \xi|
$$

and obtain a bound on the first integral:

$$
\int_{a}^{b} \int_{0}^{1}\left|\partial_{s} \xi\right|\left|\partial_{t} v_{2}\right| d t d s \leq \frac{1}{\beta} \int_{a}^{b} \int_{0}^{1} \operatorname{det} D \xi|f \circ \xi| d t d s \leq \frac{1}{\beta} \cdot\|f\|_{\infty} \cdot \mathcal{L}^{2}(\Omega) .
$$

The second part is a bit more involved. We estimate the integrand by

$\partial_{s} v_{2}=\int_{0}^{t}\left\langle\nabla f_{0} \circ \xi(\tau,),. \partial_{s} \xi(\tau,).\right\rangle d \tau \Rightarrow\left|\partial_{t} \xi\right|\left|\partial_{s} v_{2}\right| \leq \frac{1}{\beta \cdot m_{0}} \int_{0}^{1}\left|\left\langle\nabla f_{0} \circ \xi(\tau,),. \partial_{s} \xi(\tau,).\right\rangle\right| d \tau$

Because the latter is independent of $t$, we obtain

$$
\int_{a}^{b} \int_{0}^{1}\left|\partial_{t} \xi\right|\left|\partial_{s} v_{2}\right| d t d s \leq \frac{1}{\beta \cdot m_{0}} \cdot \int_{a}^{b} \int_{0}^{1}\left|\left\langle\nabla f_{0} \circ \xi(\tau, s), \partial_{s} \xi(\tau, s)\right\rangle\right| d \tau d s
$$


Now, the same argumentation that we used in the proof of Lemma $3.4 \mathrm{c}$ ) to show the integrability of $D c_{0} \circ \xi \cdot \partial_{s} \xi$ applies here for the integrability of $\left\langle\nabla f_{0} \circ \xi, \partial_{s} \xi\right\rangle$. After expanding $\nabla f_{0}$ we obtain

$$
\left\langle\nabla f_{0} \circ \xi, \partial_{s} \xi\right\rangle=\left|\partial_{t} \xi\right|\left|\partial_{s} \xi\right|\left(-\left\langle\nabla f, N^{\perp}\right\rangle+\frac{f}{\langle c, N\rangle} \cdot\left(N^{T} \cdot D c \cdot N^{\perp}+N^{\perp T} \cdot D N \cdot c\right)\right) \circ \xi .
$$

Hence, there is the integrable upper bound

$$
\left|\left\langle\nabla f_{0} \circ \xi, \partial_{s} \xi\right\rangle\right| \leq \frac{\operatorname{det} D \xi}{\beta}\left(\|\nabla f\|_{\infty}+\frac{\|f\|_{\infty}}{\beta \cdot m_{0}}(|D c|+|D N|)\right) \circ \xi
$$

which implies

$$
\int_{a}^{b} \int_{0}^{1}\left|\partial_{t} \xi\right|\left|\partial_{s} v_{2}\right| d t d s \leq \frac{\|\nabla f\|_{\infty}}{\beta^{2} m_{0}} \mathcal{L}^{2}(\Omega)+\frac{\|f\|_{\infty}}{\beta^{3} m_{0}^{2}}\left(\|D c\|_{L^{1}(\Omega)}+\|D N\|_{L^{1}(\Omega)}\right) .
$$

Putting together the partial results we firstly get a bound on the $L^{1}$-norm of $\nabla u_{2}$

$$
\left\|\nabla u_{2}(x)\right\|_{L^{1}(\Omega)} \leq \frac{1}{\beta} \cdot\|f\|_{\infty} \cdot \mathcal{L}^{2}(\Omega)+\frac{\|\nabla f\|_{\infty}}{\beta^{2} m_{0}} \mathcal{L}^{2}(\Omega) \quad+\frac{\|f\|_{\infty}}{\beta^{3} m_{0}^{2}}\left(\|D c\|_{L^{1}(\Omega)}+\|D N\|_{L^{1}(\Omega)}\right),
$$

and secondly learn that the total variation $|D u|(\Omega \backslash \Sigma)$ is bounded by $M_{\Omega \backslash \Sigma}$.

By (3.18) we have for the $L^{\infty}$-norm of $u$

$$
\|u\|_{L^{\infty}(\Omega)} \leq\left\|u_{0}\right\|_{L^{\infty}(\partial \Omega)}+\left\|T_{0}\right\|_{\infty}\left\|f_{0}\right\|_{\infty} \leq\left\|u_{0}\right\|_{L^{\infty}(\partial \Omega)}+\frac{\|f\|_{\infty}}{\beta \cdot m_{0}} .
$$

b) Now, we want to view $u$ as a $B V$-function on the domain $\Omega$. Thus, test functions are in $C_{\mathcal{c}}^{1}(\Omega)^{2}$, and thus, we have to study the limit $\lambda \rightarrow 1$ in (3.23). In part a) we already have bounds on the total variation of the components concerning $c_{0}^{\perp}(x) \cdot \mu$ and $\nabla u_{2}(x) \cdot \mathcal{L}^{2}$, which do not depend on $\lambda$. Hence, these bounds stay the same, and we can focus on the remainder

$$
\int_{\Omega}\langle\varphi(x),-N(x)\rangle u(x) d \mathcal{H}^{1}\left\llcorner\lambda(x)=\int_{a}^{b}\left\langle\psi(\lambda, s), \partial_{s} \xi^{\perp}(\lambda, s)\right\rangle v(\lambda, s) d s .\right.
$$

In Lemma 3.4 b) we introduced a partition of the interval $I$,

$$
I=\bigcup_{k=1}^{n}\left(J_{k,+} \cup J_{k,-}\right) \cup J
$$

such that $\left.\xi(1,)\right|_{.J_{k,+}}$ and $\left.\xi(1,)\right|_{.J_{k,-}}$ are both regular parametrizations of $\Sigma_{k}^{\circ}$, one for the plusside and one for the minus-side of $\Sigma_{k}^{\circ}$. If $s \in J$ then the characteristic $\xi(\lambda, s)$ hits a terminal-, branching- or kink-node of $\Sigma$, as $\lambda$ tends to 1 . Let $z_{1}, \ldots, z_{m}$ denote all these nodes, then we partition $J$ into a collection $J_{z_{1}}, \ldots, J_{z_{m}}$ by defining $J_{z_{l}}:=\left\{s \in J: \xi(\lambda, s) \rightarrow z_{l}\right.$ as $\left.\lambda \rightarrow 1\right\}$. Accordingly, we decompose the integral

$$
\int_{a}^{b}\left\langle\psi(\lambda, s), \partial_{s} \xi^{\perp}(\lambda, s)\right\rangle v(\lambda, s) d s=\sum_{l=1}^{m} \int_{J_{z_{l}}} \ldots d s+\sum_{k=1}^{n}\left(\int_{J_{k,+}} \ldots d s+\int_{J_{k,-}} \ldots d s\right) .
$$

For $J_{z_{l}}$-contributions, we have the estimate

$$
\left|\int_{J_{z_{l}}}\left\langle\psi(\lambda, s), \partial_{s} \xi^{\perp}(\lambda, s)\right\rangle v(\lambda, s) d s\right|=\left|\int_{\xi\left(\lambda, J_{z_{l}}\right)}\langle\varphi, N\rangle u d \mathcal{H}^{1}(x)\right| \leq\|\varphi\|_{\infty}\|u\|_{L^{\infty}(\Omega)} \mathcal{H}^{1}\left(\xi\left(\lambda, J_{z_{l}}\right)\right) .
$$


Because the curve-arc $\xi\left(\lambda, J_{z_{l}}\right)$ degenerates to the single point $z_{l}$ (see Figure 3.1), its length $\mathcal{H}^{1}\left(\xi\left(\lambda, J_{z_{l}}\right)\right)$ tends to zero. Hence, the contribution of those summands vanishes.

For the remaining summands we perform only those which go along $\xi\left(\lambda, J_{k,+}\right)$, since the same argumentation applies to the others which go along $\xi\left(\lambda, J_{k,-}\right)$. By Lemma $3.4 \mathrm{~b}$ ) we know that $\xi(1,):. J_{k,+} \rightarrow \Sigma_{k}^{\circ}$ is a regular parametrization and its tangent is given by the limit

$$
\partial_{s} \xi(1, s)=\lim _{\lambda \rightarrow 1} \partial_{s} \xi(\lambda, s) \quad s \in J_{k,+} .
$$

By Requirement 2.5 part 2 the field of normals extends to $\stackrel{\circ}{\Sigma}_{k}$ that means

$$
\lim _{\lambda \rightarrow 1}-N \circ \xi(\lambda, s)=n_{k} \circ \xi(1, s) \quad s \in J_{k,+} .
$$

Finally, we define the extension $u$ onto $\stackrel{\circ}{\Sigma}_{k}$ by using the extensions of $\xi^{-1}(x)$ and $s(x)$ from Lemma $3.4 \mathrm{~d})$. Let $z \in \Sigma_{k}^{\circ}$, with corresponding $s^{+}(z) \in J_{k,+}$, then we set

$$
u_{k}^{+}(z):=v\left(1, s^{+}(z)\right) \text {. }
$$

Conversely, we have $u_{k}^{+} \circ \xi(1, s)=v\left(1, s^{+}(\xi(1, s))\right)=v(1, s)$ for all $s \in J_{k,+}$. Now, we can turn to the limit. For abbreviation let $h(\lambda, s):=(\langle\varphi,-N\rangle \cdot u) \circ \xi(\lambda, s)$, then

$$
\begin{aligned}
& \mid \int_{\xi\left(\lambda, J_{k,+}\right)}\langle\varphi,-N\rangle \cdot u d \mathcal{H}^{1}(x)-\int_{\Omega}\left\langle\varphi, n_{k}\right\rangle \cdot u_{k}^{+} d \mathcal{H}^{1}\left\llcorner\Sigma_{k}(x) \mid\right. \\
& =\left|\int_{J_{k,+}} h(\lambda, s) \cdot\right| \partial_{s} \xi(\lambda, s)\left|d s-\int_{J_{k,+}} h(1, s) \cdot\right| \partial_{s} \xi(1, s)|d s| \\
& \leq \int_{J_{k,+}}|h(1, s)-h(\lambda, s)| \cdot\left|\partial_{s} \xi(1, s)\right|+|| \partial_{s} \xi(1, s)|-| \partial_{s} \xi(\lambda, s)|| \cdot|h(\lambda, s)| d s \\
& \leq \int_{J_{k,+}}|h(1, s)-h(\lambda, s)| \cdot\left|\partial_{s} \xi(1, s)\right| d s+\left\|h \circ \xi^{-1}\right\|_{L^{\infty}(\Omega)} \int_{J_{k,+}}|| \partial_{s} \xi(1, s)|-| \partial_{s} \xi(\lambda, s) \| d s .
\end{aligned}
$$

By the extensions of $N$ and $u$ the product $|h(1, s)-h(\lambda, s)| \cdot\left|\partial_{s} \xi(1, s)\right|$ tends to zero for every $s \in J_{k,+}$. Furthermore, by Lemma 3.4 part c) and the subsequent remark we know that the following bound is integrable

$$
|h(1, s)-h(\lambda, s)| \cdot\left|\partial_{s} \xi(1, s)\right| \leq 2\left\|h \circ \xi^{-1}\right\|_{L^{\infty}(\Omega)} \cdot\left|\partial_{s} \xi(1, s)\right| .
$$

Thus, the corresponding integral vanishes in the limit by dominated convergence. By same argumentation the second integral tends to zero too.

Summarizing, this means

$$
\begin{aligned}
\int_{\xi\left(\lambda, J_{k,+}\right)}\langle\varphi,-N\rangle \cdot u d \mathcal{H}^{1}(x) & \rightarrow \int_{\Omega}\left\langle\varphi, n_{k}\right\rangle \cdot u_{k}^{+} d \mathcal{H}^{1}\left\llcorner\Sigma_{k}(x),\right. \\
\int_{\xi\left(\lambda, J_{k,-}\right)}\langle\varphi,-N\rangle \cdot u d \mathcal{H}^{1}(x) & \rightarrow \int_{\Omega}\left\langle\varphi,-n_{k}\right\rangle \cdot u_{k}^{-} d \mathcal{H}^{1}\left\llcorner\Sigma_{k}(x),\right.
\end{aligned}
$$

as $\lambda \rightarrow 1$, and together we obtain the jump part for $\Sigma_{k}$

$$
\int_{\xi\left(\lambda, J_{k,+}\right) \cup \xi\left(\lambda, J_{k,-}\right)}\langle\varphi,-N\rangle \cdot u d \mathcal{H}^{1}(x) \rightarrow \int_{\Omega}\left(u_{k}^{+}-u_{k}^{-}\right)\left\langle\varphi, n_{k}\right\rangle d \mathcal{H}^{1}\left\llcorner\Sigma_{k}(x) .\right.
$$


The bound on the total variation of the jump part is

$$
\mid \int_{\Omega}\left(u_{k}^{+}-u_{k}^{-}\right)\left\langle\varphi, n_{k}\right\rangle d \mathcal{H}^{1}\left\llcorner\Sigma_{k}(x) \mid \leq 2 \cdot\|\varphi\|_{\infty} \cdot\|u\|_{L^{\infty}(\Omega)} \cdot \mathcal{H}^{1}\left(\Sigma_{k}\right) .\right.
$$

What remains to show, is that the one-sided limits $u_{k}^{+}, u_{k}^{-}$defined above are in fact the $B V$ traces $u_{\Sigma_{k}}^{+}$and $u_{\Sigma_{k}}^{-}$of $u$ on $\Sigma_{k}$. This is true, but we postpone this point to Lemma 3.7. Clearly, the complete additional jump part is given by the sum over all $k$ and the upper bound on its total variation is given by $2\|u\|_{L^{\infty}(\Omega)} \cdot \mathcal{H}^{1}(\Sigma)$. We have shown, that $u$ is in fact an element of $B V(\Omega)$ with $\|u\|_{B V(\Omega)}$ bounded by the given data, and its derivative measure reads

$$
D u=\sum_{k=1}^{n}\left(u_{k}^{+}(x)-u_{k}^{-}(x)\right) n_{k}(x) \cdot \mathcal{H}^{1}\left\llcorner\Sigma_{k}+c_{0}^{\perp}(x) \cdot \mu+\nabla u_{2}(x) \cdot \mathcal{L}^{2} .\right.
$$

So far we have seen that the candidate solution is an element of $B V(\Omega)$. Now, the following lemma shows that our candidate solution is in fact a solution of the linear PDE (3.1).

LEMMA 3.6. The candidate solution $u$ from (3.18) solves the PDE in (3.1).

Proof. By viewing $u$ as an element of $B V(\Omega \backslash \Sigma)$ we have, according to Lemma 3.5,

$$
D u=c_{0}^{\perp}(x) \cdot \mu+\nabla u_{2}(x) \cdot \mathcal{L}^{2} .
$$

By orthogonality, we obatin

$$
\left\langle c_{0}(x), D u\right\rangle=\left\langle c_{0}(x), \nabla u_{2}(x)\right\rangle \cdot \mathcal{L}^{2},
$$

and the PDE is satisfied if $\left\langle c_{0}(x), \nabla u_{2}(x)\right\rangle \cdot \mathcal{L}^{2}$ equals $f_{0}(x) \cdot \mathcal{L}^{2}$. With $\varphi \in C_{0}(\Omega)$ we test the measure $\left\langle c_{0}(x), \nabla u_{2}(x)\right\rangle \cdot \mathcal{L}^{2}$. By changing variables, $\psi=\varphi \circ \xi, v_{2}=u_{2} \circ \xi$, we obtain

$$
\begin{aligned}
& \int_{a}^{b} \int_{0}^{1} \psi\left\langle c_{0}, \nabla u_{2}\right\rangle \circ \xi \operatorname{det} D \xi d t d s=\int_{a}^{b} \int_{0}^{1} \psi\left\langle\partial_{t} \xi,\left(-\partial_{s} \xi^{\perp} \partial_{t} v_{2}+\partial_{t} \xi^{\perp} \partial_{s} v_{2}\right)\right\rangle d t d s \\
& \quad=\int_{a}^{b} \int_{0}^{1} \psi \cdot \partial_{t} v_{2} \cdot\left\langle\partial_{t} \xi,-\partial_{s} \xi^{\perp}\right\rangle d t d s=\int_{a}^{b} \int_{0}^{1} \varphi \circ \xi \cdot f_{0} \circ \xi \cdot \operatorname{det} D \xi d t d s=\int_{\Omega} \varphi f_{0} d x .
\end{aligned}
$$

Here, we used the fact that $\partial_{t} v_{2}=f_{0} \circ \xi$ from (3.19). This shows that the total variation of the difference measure is

$$
\left|\left\langle c_{0}(x), \nabla u_{2}(x)\right\rangle \cdot \mathcal{L}^{2}-f_{0}(x) \cdot \mathcal{L}^{2}\right|(\Omega)=0,
$$

and hence the measures are equal.

Next we study $B V$-traces of the candidate $u$ along level-lines of $T_{0}$. In particular we will see that $u$ satisfies the boundary condition as $B V$ boundary trace.

LEMMA 3.7. Let $u$ be the candidate solution from (3.18), then

a) (start): $u$ satisfies the boundary condition, i.e.,

$$
\lim _{r \rightarrow 0+} \frac{1}{r^{2}} \int_{\Omega \cap B_{r}(z)}\left|u_{0}(z)-u(x)\right| d x=0
$$

for every Lebesgue point $z \in \partial \Omega$ of $u_{0}$. 
b) (restart): for every $z \in \Omega \backslash \Sigma$ that corresponds to a Lebesgue point $z^{\prime}$ of $u_{0}$, that means $z^{\prime}=\gamma(s(z))$ is a Lebesgue point of $u_{0}$, we have

$$
\lim _{r \rightarrow 0+} \frac{1}{r^{2}} \int_{B_{r}^{>}(z)}|u(z)-u(x)| d x=0 \quad \text { and } \quad \lim _{r \rightarrow 0+} \frac{1}{r^{2}} \int_{B_{r}^{<}(z)}|u(z)-u(x)| d x=0 .
$$

$B_{r}^{<}(z)$ and $B_{r}^{>}(z)$, for $r$ small enough, denote the cut-off disks

$$
B_{r}^{<}(z):=\left\{x \in B_{r}(z): T_{0}(x)<T_{0}(z)\right\}, \quad B_{r}^{>}(z):=\left\{x \in B_{r}(z): T_{0}(x)>T_{0}(z)\right\} .
$$

Let $\Gamma:=\Omega_{T_{0}=\lambda}$ be a $\lambda$-level of $T_{0}$ for some $0<\lambda<1$ and let $\Gamma$ be oriented by $\left.N\right|_{\Gamma}$. If $z \in \Gamma$, the result above means that the traces $u_{\Gamma}^{+}$and $u_{\Gamma}^{-}$are identical. There is no jump across $\Gamma$, and if $z$ corresponds to a Lebesgue point of $u_{0}$, then it is itself a Lebesgue point of $u$. Owing to the identity of both traces the restriction $\left.u\right|_{\Gamma}$ is well-defined. Moreover, we have $\left.u\right|_{\Gamma} \in B V(\Gamma)$.

c) (stop): for every $z \in \Sigma_{k}^{\circ}$ that corresponds to a Lebesgue point $z^{\prime}$ of $u_{0}$ with respect to the plus-side of $\Sigma_{k}$, that means $z^{\prime}=\gamma\left(s^{+}(z)\right)$ is a Lebesgue point of $u_{0}$, we have

$$
\lim _{r \rightarrow 0+} \frac{1}{r^{2}} \int_{B_{r}^{+}(z)}\left|u_{k}^{+}(z)-u(x)\right| d x=0 .
$$

Here, $u_{k}^{+}(z)$ is defined by (3.24). $B_{r}^{+}(z)$ is the disk centered at $z$ and restricted to the plus-side. Hence, the trace $u_{\Sigma_{k}}^{+}$is given by $u_{k}^{+}$. Moreover, we have $u_{k}^{+} \in B V\left(\Sigma_{k}^{\circ}\right)$. The analogous result holds for the minus-side.

Proof.

a) \& b). Let $z \in \bar{\Omega} \backslash \Sigma$ and let $(\tau, \sigma)$ be its characteristic coordinates, i.e., $z=\xi(\tau, \sigma)$. Choose $r>0$ sufficiently small, then

$$
\frac{1}{r^{2}} \int_{B_{r}^{\succ}(z)}|u(z)-u(x)| d x=\frac{1}{r^{2}} \int_{s_{-}(r)}^{s_{+}(r)} \int_{\tau}^{t_{+}(r, s)}|v(\tau, \sigma)-v(t, s)| \operatorname{det} D \xi d t d s .
$$

Because any level-line of $T_{0}$ is a regular $C^{1}$-curve, the cut-off disk $B_{r}^{>}(z)$ tends to a half disk, aligned with the tangent $\partial_{s} \xi(\tau, \sigma)$ of this curve. Thus, the we obtain the asymptotic $s_{ \pm}(r)=$ $\sigma \pm \mathcal{O}(r)$. Since $\operatorname{det} D \xi(t, s) \neq 0$, a similar argument applies regarding the $t$-variable, i.e., $t_{+}(r, s)=\tau+\mathcal{O}(r)$. Taylor expansion yields $\partial_{s} \xi(t, s)=\partial_{s} \xi(\tau, s)+\mathcal{O}(t-\tau)$ and therefore we have

$$
\operatorname{det} D \xi(t, s) \leq\left|\partial_{t} \xi\right| \cdot\left|\partial_{s} \xi\right|=\frac{\left|\partial_{s} \xi(\tau, s)\right|}{\beta \cdot m_{0}}+\mathcal{O}(t-\tau) \leq C+\mathcal{O}(t-\tau)
$$

for a suitable constant $C$. The difference is estimated by

$$
|v(t, s)-v(\tau, \sigma)| \leq\left|\gamma^{*} u_{0}(s)-\gamma^{*} u_{0}(\sigma)\right|+\left|v_{2}(t, s)-v_{2}(\tau, \sigma)\right|,
$$

with

$$
\begin{aligned}
\left|v_{2}(t, s)-v_{2}(\tau, \sigma)\right| & =\left|\int_{0}^{t} f_{0} \circ \xi(h, s) d h-\int_{0}^{\tau} f_{0} \circ \xi(h, \sigma) d h\right| \\
& \leq \int_{0}^{t}\left|\nabla f_{0} \circ \xi\left(h, s_{*}\right)\right|\left|\partial_{s} \xi\left(h, s_{*}\right)\right||s-\sigma| d h+\left|\int_{\tau}^{t} f_{0} \circ \xi(h, \sigma) d h\right| .
\end{aligned}
$$


Because $z \notin \Sigma$, we have for $r$ that the cut-off disk $B_{r}^{>}(z)$ is contained in some lower level-set $\Omega_{T_{0} \leq \lambda}$ with $\lambda<1$. Hence, $t \leq \lambda$ for the last two integrals, and the following bound exists:

$$
M:=\left\|\nabla f_{0}\right\|_{L^{\infty}(\Omega)} \cdot \sup _{\left(h, s_{*}\right) \in[0, \lambda] \times\left[s_{-}(r), s_{+}(r)\right]}\left|\partial_{s} \xi\left(h, s_{*}\right)\right| .
$$

In summary,

$$
\begin{aligned}
|v(t, s)-v(\tau, \sigma)| & \leq\left|\gamma^{*} u_{0}(s)-\gamma^{*} u_{0}(\sigma)\right|+t \cdot M|s-\sigma|+\left\|f_{0}\right\|_{\infty}|t-\tau| \\
& \leq\left|\gamma^{*} u_{0}(s)-\gamma^{*} u_{0}(\sigma)\right|+\mathcal{O}(s-\sigma)+\mathcal{O}(t-\tau) .
\end{aligned}
$$

Armed with the last result and the estimate of the determinant, we have

$$
\begin{aligned}
\frac{1}{r^{2}} \int_{B_{r}^{>}(z)}|u(z)-u(x)| d x & \leq \frac{1}{r^{2}} \int_{s_{-}(r)}^{s_{+}(r)} \int_{\tau}^{t_{+}(r)}\left|\gamma^{*} u_{0}(s)-\gamma^{*} u_{0}(\sigma)\right| \cdot C+\mathcal{O}(s-\sigma)+\mathcal{O}(t-\tau) d t d s \\
& \leq \frac{C^{\prime}}{r} \int_{s_{-}(r)}^{s_{+}(r)}\left|\gamma^{*} u_{0}(s)-\gamma^{*} u_{0}(\sigma)\right| d s+\mathcal{O}(r) .
\end{aligned}
$$

The latter expression tends, as $r \rightarrow 0$, to zero for any Lebesgue-point $\sigma$ of $\gamma^{*} u_{0}$, i.e., for any Lebesgue-point $z^{\prime}=\gamma(\sigma)$ of $u_{0}$. If $z \in \partial \Omega$, then $\Omega \cap B_{r}(z)=B_{r}^{>}(z)$ and the argumentation above shows that $u$ satisfies the boundary condition as $B V$-trace. If $z \in \Omega \backslash \Sigma$, we have got the assertion for the $B_{r}^{>}(z)$-case. In the $B_{r}^{<}(z)$-case the one and only difference is that, after having changed variables, we have to integrate the $t$-variable over the interval $\left[t_{-}(r, s), \tau\right]$. For the remainder one must perform the same steps to get the assertion for the $B_{r}^{<}(z)$. Finally, the restriction $\left.u\right|_{\Gamma}$ is defined, and when parametrizing $\Gamma$ regularly by $\xi_{\lambda}(s):=\xi(\lambda, s)$, we have

$$
\xi_{\lambda}^{*} u(s)=u \circ \xi(\lambda, s)=v(\lambda, s)=\gamma^{*} u_{0}(s)+v_{2}(\lambda, s) \quad s \in \mathbb{R} .
$$

Since $v_{2}(\lambda, s)$ is a periodic $C^{1}$-function, while $\gamma^{*} u_{0}(s)$ is a periodic $B V$-function, the sum $\xi_{\lambda}^{*} u$ is a periodic $B V$-function, and consequently $\left.u\right|_{\Gamma} \in B V(\Gamma)$.

c) In order to argue in the same way as in part a) \& b) we cannot use diffeomorphism $\xi$, because $\left.c_{0}\right|_{\Sigma}=0$ and thus $\operatorname{det} D \xi(1, s)=0$. But at least $\xi_{1}(s):=\xi(1, s)$ with $s \in J_{k,+}$ is a regular parametrization of $\Sigma_{k}^{\circ}$ (cf. Lemma 3.4 b)), so we set up a local diffeomorphism by considering the unscaled backward IVP

$$
y^{\prime}=-c(y), \quad y(0, s)=\xi(1, s), \quad s \in J_{k,+}
$$

with $c$ extended onto $\stackrel{\circ}{\Sigma}_{k}$ by $c^{+}$. Let $\tau(s)=\tau(1, s)$ denote the time when $y(., s)$ reaches the boundary $\partial \Omega$. In the proof of Lemma $3.4 \mathrm{~b}$ ) we have seen that $\tau: J_{k,+} \rightarrow \mathbb{R}$ is continuously differentiable. For $s \in J_{k,+}$ we have

$$
u \circ y(t, s)=\gamma^{*} u_{0}(s)+\int_{t}^{\tau(s)} f \circ y(h, s) d h, \quad u_{k}^{+} \circ \xi(1, s)=u_{k}^{+} \circ y(0, s)=\lim _{t \rightarrow 0} u \circ y(t, s) .
$$

Let then $z \in \stackrel{\circ}{\Sigma}_{k}$ with $z=\xi_{1}(\sigma), \sigma \in J_{k,+}$. For small $r$, we obtain

$$
\frac{1}{r^{2}} \int_{B_{r}^{+}(z)}\left|u_{k}^{+}(z)-u(x)\right| d x=\frac{1}{r^{2}} \int_{s_{-}(r)}^{s_{+}(r)} \int_{0}^{t(r, s)}\left|u_{k}^{+} \circ y(0, \sigma)-u \circ y(t, s)\right||\operatorname{det} D y| d t d s
$$

by changing variables. For $r$ small enough, the determinant $|\operatorname{det} D y(t, s)|$ is approximately $|\operatorname{det} D y(0, \sigma)|$ with

$$
|\operatorname{det} D y(0, \sigma)|=\left|\partial_{s} \xi_{1}(\sigma)\right|\left|c^{+}(z)\right|\left|\left\langle c^{+}(z), n_{k}(z)\right\rangle\right| \neq 0
$$


which is non-zero because of Requirement 2.6 part 2c). For the functions $s_{+}(r), s_{-}(r)$, and $t(r, s)$ we have the same asymptotic, for $r \rightarrow 0$, as in the previous part.

Let $\tau_{1}=\min (\tau(s), \tau(\sigma))$ and $\tau_{2}=\max (\tau(s), \tau(\sigma))$. If $\tau_{1}=\tau(s)$, we set $s_{1}=\sigma$, and otherwise we set $s_{1}=s$. For the difference $\left|u_{k}^{+} \circ y(0, \sigma)-u \circ y(t, s)\right|$ we estimate first

$$
\begin{aligned}
& \left|\int_{0}^{\tau(\sigma)} f \circ y(h, \sigma) d h-\int_{t}^{\tau(s)} f \circ y(h, s) d h\right| \\
& \leq\left|\int_{\tau_{1}}^{\tau_{2}} f \circ y\left(h, s_{1}\right) d h\right|+\left|\int_{t}^{\tau_{1}} f \circ y(h, \sigma)-f \circ y(h, s) d h\right|+\left|\int_{0}^{t} f \circ y(h, \sigma) d h\right| \\
& \leq\|f\|_{\infty} \cdot|\tau(\sigma)-\tau(s)|+\|\nabla f\|_{\infty} \cdot\left\|\partial_{s} y\left(., s^{*}\right)\right\|_{\infty} \cdot \tau_{1} \cdot|s-\sigma|+\|f\|_{\infty} t \\
& \leq\left(\|f\|_{\infty} \cdot\left|\partial_{s} \tau\left(s^{*}\right)\right|+\|\nabla f\|_{\infty} \cdot\left\|\partial_{s} y\left(., s^{*}\right)\right\|_{\infty} \cdot \tau_{1}\right) \cdot|s-\sigma|+\|f\|_{\infty} t .
\end{aligned}
$$

Because $\tau(s)$ and $\tau(\sigma)$ are arc-lengths of characteristics, $\tau_{1}$ is bounded by $1 /\left(\beta \cdot m_{0}\right)$ according to Lemma 3.1. And, as highlighted in the proof of Lemma $3.4 \mathrm{~b}$ ), when $s$ is restricted to small neighborhood around $\sigma \in J_{k,+}$, we also have uniform bounds on $\left|\partial_{s} \tau\left(s^{*}\right)\right|$ and $\left\|\partial_{s} y\left(., s^{*}\right)\right\|_{\infty}$. Taking this into account, the upper bound in (3.25) is $\mathcal{O}(s-\sigma)+\mathcal{O}(t)$ and consequently

$$
\left|u_{k}^{+} \circ y(0, \sigma)-u \circ y(t, s)\right|=\left|\gamma^{*} u_{0}(\sigma)-\gamma^{*} u_{0}(s)\right|+\mathcal{O}(s-\sigma)+\mathcal{O}(t) .
$$

As in part a) \& b), we obtain

$$
\frac{1}{r^{2}} \int_{B_{r}^{+}(z)}\left|u_{k}^{+}(z)-u(x)\right| d x \leq \frac{C^{\prime}}{r} \int_{s_{-}(r)}^{s_{+}(r)}\left|\gamma^{*} u_{0}(s)-\gamma^{*} u_{0}(\sigma)\right| d s+\mathcal{O}(r)
$$

which tends to zero whenever $z$, by $\gamma(\sigma)=\gamma\left(s^{+}(z)\right)$, corresponds to a Lebesgue point of $u_{0}$. From the representation

$$
u_{k}^{+} \circ \xi(1, s)=\gamma^{*} u_{0}(s)+\int_{0}^{1} f_{0} \circ \xi(\tau, s) d \tau, \quad s \in J_{k,+},
$$

it is obvious that $u_{k}^{+} \circ \xi(1, s)$ is a $B V$-function of one variable on the interval $J_{k,+}$ and that $u_{k}^{+} \in B V\left(\Sigma_{k}^{\circ}\right)$ since $\xi(1, s)$ is regular.

Remark: part b) of Lemma 3.7 is called "restart" because having stopped the characteristics at some intermediate $\lambda$-level $\Gamma$ of $T_{0}$, the restarted problem

$$
\langle c(x), D w\rangle=f(x) \cdot \mathcal{L}^{2} \quad \text { in } \quad \Omega_{T_{0}>\lambda},\left.\quad w\right|_{\Gamma}=\left.u\right|_{\Gamma} .
$$

is of the same type as (3.1). This is, because Lemma $3.7 \mathrm{~b}$ ) ensures that $\left.u\right|_{\Gamma} \in B V(\Gamma)$. Moreover, when applying the same method of construction, then $w$ reproduces $u$ by $w=\left.u\right|_{\Omega_{T_{0}>\lambda}}$.

3.3. Uniqueness and Stability. We have seen that the linear problem (3.1) has a solution in $B V(\Omega)$. In this section we carry on with the uniqueness of the solution and its continuous dependence on the data.

THEOREM 3.8. The solution of the linear problem (3.1) is

a) unique and stable regarding perturbations of the boundary data: let $u$ and $w$ be solutions of PDE (3.1)

in $\Omega \backslash \Sigma$ such that $\left.u\right|_{\partial \Omega}=u_{0}$ and $\left.w\right|_{\partial \Omega}=w_{0}$, then

$$
\|u-w\|_{L^{\infty}(\Omega)}=\left\|u_{0}-w_{0}\right\|_{L^{\infty}(\partial \Omega)} .
$$


b) stable regarding perturbations of the right-hand side of PDE (3.1): let $u$ and $w$ be the solutions of

$$
\langle c(x), D u\rangle=f(x) \cdot \mathcal{L}^{2}, \quad\langle c(x), D w\rangle=g(x) \cdot \mathcal{L}^{2},
$$

in $\Omega \backslash \Sigma$ with the same boundary data $\left.u\right|_{\partial \Omega}=\left.w\right|_{\partial \Omega}=u_{0}$, then

$$
\|u-w\|_{L^{\infty}(\Omega)} \leq \frac{\|f-g\|_{\infty}}{\beta \cdot m_{0}} .
$$

Proof.

a) By the linearity of the scaled PDE the difference $h:=u-w$ clearly satisfies the homogeneous

$$
\left\langle c_{0}(x), D h\right\rangle=0 \quad \text { in } \Omega \backslash \Sigma,\left.\quad h\right|_{\partial \Omega}=h_{0}:=\left(u_{0}-w_{0}\right) .
$$

In the following passage we prove that the latter problem has a unique solution. Let $\varphi \in$ $C_{c}^{1}(\Omega \backslash \Sigma)$. We set $\psi=\varphi \circ \xi$ and $v=h \circ \xi$. Moreover, let $\tilde{\Omega}:=\Omega \backslash(\Sigma \cup S)$ as in Lemma 3.3.

Next, we want to rewrite the PDE in characteristic variables. If we change the variables first and compute the $B V$-derivative of $v$ afterwards, we obtain

$$
\begin{aligned}
\int_{\tilde{\Omega}} h \partial_{k} \varphi d x & =\int_{\xi^{-1}(\tilde{\Omega})} v \partial_{k} \varphi \circ \xi \operatorname{det} D \xi d(t, s)=\int_{\xi^{-1}(\tilde{\Omega})} v(-1)^{l}\left(\partial_{t}\left(\partial_{s} \xi_{l} \cdot \psi\right)-\partial_{s}\left(\partial_{t} \xi_{l} \cdot \psi\right)\right) d(t, s) \\
& =-\int_{\xi^{-1}(\tilde{\Omega})} \psi \cdot(-1)^{l}\left(\partial_{s} \xi_{l} d D_{t} v(t, s)-\partial_{t} \xi_{l} d D_{s} v(t, s)\right) .
\end{aligned}
$$

And, if we proceed the other way round, meaning we first compute the $B V$-derivative of $u$ and then pull back onto characteristic variables, we obtain

$$
\int_{\tilde{\Omega}} h \partial_{k} \varphi d x=-\int_{\tilde{\Omega}} \varphi d D_{k} h(x)=-\int_{\tilde{\Omega}} \psi \circ \xi^{-1} d D_{k} h(x)=-\int_{\xi^{-1}(\tilde{\Omega})} \psi d \xi_{\sharp}^{-1} D_{k} h(t, s) .
$$

Hence, we have $\xi_{\sharp}^{-1} D_{k} h=(-1)^{l}\left(\partial_{s} \xi_{l} D_{t} v-\partial_{t} \xi_{l} D_{s} v\right)$ and employing matrix-vector notation, this can be written as

$$
\xi_{\sharp}^{-1} D h=\left(-\partial_{s} \xi^{\perp} \mid \partial_{t} \xi^{\perp}\right) D_{t, s} v=\operatorname{det} D \xi \cdot D \xi^{-T} D_{t, s} v \quad \Rightarrow \quad D_{t, s} v=\frac{D \xi^{T}}{\operatorname{det} D \xi} \cdot \xi_{\sharp}^{-1} D h .
$$

The latter equality yields a chain rule. Note that in the special case of $D h=\nabla h(x) \cdot \mathcal{L}^{2}$ being absolutely continuous one gets back the well known chain rule:

$$
\xi_{\sharp}^{-1} D h=\nabla h \circ \xi \cdot \xi_{\sharp}^{-1}\left(\mathcal{L}^{2}\llcorner\tilde{\Omega})=\nabla h \circ \xi \cdot \operatorname{det} D \xi \cdot \mathcal{L}^{2}\left\llcorner\xi^{-1}(\tilde{\Omega}) .\right.\right.
$$

From (3.26) we read off

$$
\operatorname{det} D \xi \cdot D_{t} v=\left\langle\partial_{t} \xi_{,} \xi_{\sharp}^{-1} D h\right\rangle=\left\langle c_{0} \circ \xi_{,} \xi_{\sharp}^{-1} D h\right\rangle=\xi_{\sharp}^{-1}\left(\left\langle c_{0}, D h\right\rangle\right)=0,
$$

and hence the homogeneous PDE in characteristic variables is simply $D_{t} v=0$. Since $v$ is a $B V$ function on $] 0,1[\times] a, b\left[=\xi^{-1}(\tilde{\Omega})\right.$, there exist slices $v_{s}(t):=v(t, s)$ for almost every $\left.s \in\right] a, b[$ (see [4, Chapter 3.11]). By theorem [4, Theorem 3.107] such a slice $v_{s}$ is itself a $B V$-function of one variable on ], $1[$ and its derivative relates to the partial derivative of $v$ by

$$
D_{t} v=\left(\mathcal{L}^{1}\llcorner] a, b[) \otimes D v_{s} .\right.
$$

The PDE $D_{t} v=0$ clearly implies that the derivative of every slice is zero and therefore the slice $v_{s}$ is equivalent to a constant $v(t, s)=\alpha(s)$ at most depending on $s$. Finally, $\alpha(s)$ is fixed by 
the boundary condition. Let $z \in \partial \Omega$ with $z=\gamma(\sigma)$. Proceeding as in the proof of Lemma 3.7 a), we write down the integral for the boundary trace as

$$
\frac{1}{r^{2}} \int_{\Omega \cap B_{r}(x)}\left|h(y)-h_{0}(z)\right| d y=\frac{C^{\prime}}{r} \int_{s_{-}(r)}^{s_{+}(r)}\left|\alpha(s)-\gamma^{*} h_{0}(\sigma)\right| d s+\mathcal{O}(r) .
$$

Thus, in order to satisfy the boundary condition the only possible choice is $\alpha(\sigma)=\gamma^{*} h_{0}(\sigma)$ whenever $\sigma \in] a, b\left[\right.$ is a Lebesgue-point of $\gamma^{*} h_{0}$. In summary, the solution of the homogeneous problem is given by

$$
v(t, s)=\gamma^{*} h_{0}(s) \quad \Rightarrow \quad h(x)=h_{0}\left(\eta\left(T_{0}(x), x\right)\right),
$$

which further implies the stability equation

$$
\|u-w\|_{L^{\infty}(\Omega)}=\|h\|_{L^{\infty}(\Omega)}=\left\|h_{0}\right\|_{L^{\infty}(\partial \Omega)}=\left\|u_{0}-w_{0}\right\|_{L^{\infty}(\partial \Omega)},
$$

The solution $u$ is thus unique in the case of $w_{0}=u_{0}$.

b) Here again, we consider the equivalent scaled problems

$$
\left\langle c_{0}(x), D u\right\rangle=f_{0}(x) \cdot \mathcal{L}^{2}, \quad\left\langle c_{0}(x), D w\right\rangle=g_{0}(x) \cdot \mathcal{L}^{2} .
$$

By using the uniqueness result from part a), we can write down the solutions as

$$
u(x)=u_{0}\left(\eta\left(T_{0}(x), x\right)\right)+\int_{0}^{T_{0}(x)} f_{0} \circ \eta(\tau, x) d \tau, \quad w(x)=u_{0}\left(\eta\left(T_{0}(x), x\right)\right)+\int_{0}^{T_{0}(x)} g_{0} \circ \eta(\tau, x) d \tau .
$$

The difference of the solutions is easily estimated by

$$
|u(x)-w(x)| \leq\left\|f_{0}-g_{0}\right\|_{\infty} \cdot T_{0}(x) \leq \frac{\|f-g\|_{\infty}}{\beta \cdot m_{0}},
$$

taking the essential supremum on the left-hand side yields the assertion.

Having the uniqueness of the solution and the continuous dependence on the right-hand side as well as on the boundary data, the last theorem of this section focuses on the continuous dependence of the solution on the transport field and the time function.

THEOREM 3.9. Let $\left(c_{n}\right)_{n \in \mathbb{N}}$ be a sequence of transport fields and let $c$ be a transport field. Let $\left(f_{n}\right)_{n \in \mathbb{N}}$ be a sequence of right-hand sides and let $f$ be a right-hand side. Let $\left(T_{n}\right)_{n \in \mathbb{N}}$ be a sequence of time functions and let $T$ be a time function with corresponding fields of normals $N_{n}=\nabla T_{n} /\left|\nabla T_{n}\right|$ and $N=\nabla T /|\nabla T|$. Consider the family of linear problems

$$
\begin{aligned}
& \left\langle c_{n}(x), D u_{n}\right\rangle=f_{n},\left.\quad u_{n}\right|_{\partial \Omega}=u_{0}, \quad\left\langle c_{n}(x), N_{n}(x)\right\rangle \geq \beta, \\
& \langle c(x), D u\rangle=f,\left.\quad u\right|_{\partial \Omega}=u_{0}, \quad\langle c(x), N(x)\rangle \geq \beta,
\end{aligned}
$$

in $\Omega \backslash \Sigma_{n}$ respectively $\Omega \backslash \Sigma$. If the following requirements hold

1. $f_{n}$ converges uniformly to $f:\left\|f_{n}-f\right\|_{\infty} \rightarrow 0$,

2. $\nabla f_{n}$ is bounded: $\left\|\nabla f_{n}\right\|_{\infty} \leq M_{1}$ for all $n$,

3. $c_{n}$ converges uniformly to $c:\left\|c_{n}-c\right\|_{\infty} \rightarrow 0$,

4. $D c_{n}$ is bounded: $\left\|D c_{n}\right\|_{L^{1}(\Omega)} \leq M_{2}$ for all $n$,

5. $T_{n}$ converges uniformly to $T:\left\|T_{n}-T\right\|_{\infty} \rightarrow 0$,

6. $\nabla T_{n}$ converges uniformly to $\nabla T:\left\|\nabla T_{n}-\nabla T\right\|_{\infty} \rightarrow 0$,

7. $D N_{n}$ is bounded: $\left\|D N_{n}\right\|_{L^{1}(\Omega)} \leq M_{3}$ for all $n$,

8. lengths of stops sets $\Sigma_{n}$ are uniformly bounded: $\mathcal{H}^{1}\left(\Sigma_{n}\right) \leq M_{4}$ for all $n$, 
9. there is a positive lower bound on

$$
\min \left\{m_{0}, \inf _{n \in \mathbb{N}} m_{n, 0}\right\} \geq \bar{m}>0,
$$

where $m_{n, 0}, m_{0}$ denote the positive minima of the transformed time functions $T_{n, 0}, T_{0}$ from Lemma 3.1, then the sequence of solutions $u_{n}$ converges $B V$ weakly* to $u$, i.e.,

$$
u_{n} \stackrel{*}{\rightarrow} u \text { in } B V(\Omega) \text {, as } n \rightarrow \infty .
$$

Proof. $L^{1}$-convergence. We begin with showing the $L^{1}$-convergence of $u_{n}$ to $u$. Again, we use the scaled PDEs

$$
\left\langle c_{n, 0}(x), D u_{n}\right\rangle=f_{n, 0} \quad \text { and } \quad\left\langle c_{0}(x), D u\right\rangle=f_{0},
$$

where the first PDE was scaled with $1 /\left\langle c_{n}, \nabla T_{n, 0}\right\rangle$ and the second one with $1 /\left\langle c, \nabla T_{0}\right\rangle$. We denote the backward characteristics associated with the transport fields $c_{n, 0}$ by $\eta_{n}$. Likewise, $\eta$ denotes the characteristics corresponding to $c_{0}$. The solutions are then given by

$$
\begin{aligned}
& u_{n}(x)=u_{1, n}(x)+u_{2, n}(x)=u_{0}\left(\eta_{n}\left(T_{0}(x), x\right)\right)+\int_{0}^{T_{n, 0}(x)} f_{n, 0} \circ \eta_{n}(\tau, x) d \tau, \\
& u(x)=u_{1}(x)+u_{2}(x)=u_{0}\left(\eta\left(T_{0}(x), x\right)\right)+\int_{0}^{T_{0}(x)} f_{0} \circ \eta(\tau, x) d \tau .
\end{aligned}
$$

with an additive decomposition as in (3.19). We split the proof into three steps.

Step one. We restrict the discussion to a lower level set $\Omega_{T_{0} \leq \lambda}$. By Lemma 3.5 we know that the solutions are bounded by

$$
\left\|u_{n}\right\|_{L^{\infty}(\Omega)} \leq\left\|u_{0}\right\|_{L^{\infty}(\partial \Omega)}+\frac{\left\|f_{n}\right\|_{\infty}}{\beta \cdot m_{n, 0}} \leq\left\|u_{0}\right\|_{L^{\infty}(\partial \Omega)}+\frac{M}{\beta \cdot \bar{m}}=: C_{1},
$$

where $M$ denotes the $L^{\infty}$-bound of the family $\left\{f_{n}, f\right\}$. The same estimate is true for $\|u\|_{L^{\infty}(\Omega)} \leq$ $C_{1}$. Now, we choose $\lambda$ such that $\left\|u_{n}\right\|_{L^{1}\left(\Omega \backslash \Omega_{T_{0} \leq \lambda}\right)} \leq C_{1} \cdot \mathcal{L}^{2}\left(\Omega \backslash \Omega_{T_{0} \leq \lambda}\right) \leq \varepsilon / 6$ and obtain

$$
\left\|u_{n}-u\right\|_{L^{1}(\Omega)} \leq\left\|u_{n}-u\right\|_{L^{1}\left(\Omega_{T_{0} \leq \lambda}\right)}+2 C_{1} \cdot \mathcal{L}^{2}\left(\Omega \backslash \Omega_{T_{0} \leq \lambda}\right) \leq\left\|u_{n}-u\right\|_{L^{1}\left(\Omega_{T_{0} \leq \lambda}\right)}+\frac{\varepsilon}{3} .
$$

For the remainder of the proof we focus on $\left\|u_{n}-u\right\|_{L^{1}\left(\Omega_{T_{0} \leq \lambda}\right)}$. Moreover, we choose $n_{0} \in \mathbb{N}$ such that for $n \geq n_{0}$ each stop set $\Sigma_{n}$ (corresponding to $T_{n}$ ) is compactly contained in $\Omega \backslash \Omega_{T_{0} \leq \lambda}$. This is possible because $T_{n}$ converges uniformly to $T$. From now on we consider only the subsequences starting at index $n_{0}$. So, we can be sure that the point $x \in \Omega_{T_{0} \leq \lambda}$ does not belong to one of the singular sets $\Sigma_{n}$. Hence, all curves $\eta_{n}(., x)$ are well-defined. In addition, we have that $T_{n, 0}$ converges to $T_{0}$ and that $\nabla T_{n, 0}$ converges to $\nabla T_{0}$ both uniformly on $\Omega_{T_{0} \leq \lambda}$.

Step two. We study the contribution $\left\|u_{2, n}-u_{2}\right\|_{L^{1}\left(\Omega_{T_{0}} \leq \lambda\right)}$. Let $a(x):=\min \left\{T_{0}(x), T_{n, 0}(x)\right\}$, then we have following pointwise estimate

$$
\left|u_{2, n}(x)-u_{2}(x)\right| \leq \int_{0}^{a(x)}\left|f_{n, 0} \circ \eta_{n}(\tau, x)-f_{0} \circ \eta(\tau, x)\right| d \tau+\frac{M}{\beta \cdot \bar{m}}\left|T_{n, 0}(x)-T_{0}(x)\right| .
$$

Now, we make $M /(\beta \cdot \bar{m})\left\|T_{n, 0}-T_{0}\right\|_{L^{\infty}\left(\Omega_{T_{0} \leq \lambda}\right)} \mathcal{L}^{2}\left(\Omega_{T_{0} \leq \lambda}\right) \leq \varepsilon / 6$ by using the locally uniform convergence of $T_{n, 0}$ to $T_{0}$, and find

$$
\left\|u_{2, n}-u_{2}\right\|_{L^{1}\left(\Omega_{T_{0} \leq \lambda}\right)} \leq \int_{\Omega_{T_{0} \leq \lambda}} \int_{0}^{\lambda}\left|f_{0} \circ \eta(\tau, x)-f_{0, n} \circ \eta_{n}(\tau, x)\right| \cdot \mathbb{1}_{] 0, a(x)[}(\tau) d \tau d x+\frac{\varepsilon}{6} .
$$


The remaining integral will become smaller than $\varepsilon / 6$ by dominated convergence since the integrand possesses the integrable bound $\left|f_{0} \circ \eta-f_{0, n} \circ \eta_{n}\right| \cdot \mathbb{1}_{0, a(x)[} \leq 2 M /(\beta \cdot \bar{m})$ and converges pointwise almost everywhere to zero. In combination with step one, we end up with

$$
\left\|u_{n}-u\right\|_{L^{1}\left(\Omega_{T_{0} \leq \lambda}\right)} \leq\left\|u_{1, n}-u_{1}\right\|_{L^{1}\left(\Omega_{T_{0} \leq \lambda}\right)}+\left\|u_{2, n}-u_{2}\right\|_{L^{1}\left(\Omega_{T_{0} \leq \lambda}\right)}+\frac{\varepsilon}{3} \leq\left\|u_{1, n}-u_{1}\right\|_{L^{1}\left(\Omega_{T_{0} \leq \lambda}\right)}+\frac{2 \varepsilon}{3} .
$$

Step three. We study the contribution $\left\|u_{1, n}-u_{1}\right\|_{L^{1}\left(\Omega_{T_{0} \leq \lambda}\right)}$. For now, we assume that the boundary data is continuous. Then we can appeal to dominated convergence again to make

$$
\left\|u_{1, n}-u_{1}\right\|_{L^{1}\left(\Omega_{T_{0} \leq \lambda}\right)}=\int_{\Omega_{T_{0} \leq \lambda}}\left|u_{0}\left(\eta_{n}\left(T_{n, 0}(x), x\right)\right)-u_{0}\left(\eta\left(T_{0}(x), x\right)\right)\right| d x,
$$

small given that $\eta_{n}\left(T_{n, 0}(x), x\right)$ converges pointwise to $\eta\left(T_{0}(x), x\right)$. The difference $\eta_{n}(., x)-$ $\eta(., x)$ of the characteristics satisfies the IVP

$$
\left(\eta_{n}-\eta\right)^{\prime}=c_{0}(\eta)-c_{n, 0}\left(\eta_{n}\right), \quad\left(\eta_{n}-\eta\right)(0, x)=0 .
$$

Hence, for $t \leq a(x)$, integration yields

$$
\left(\eta_{n}-\eta\right)(t, x)=\int_{0}^{t} c_{0}(\eta(\tau, x))-c_{n, 0}\left(\eta_{n}(\tau, x)\right) d \tau .
$$

Next, we supplement $c_{0}\left(\eta_{n}(\tau, x)\right)$ and use the triangle inequality to get a first estimate

$$
\left|\eta_{n}-\eta\right|(t, x) \leq \int_{0}^{t}\left|c_{0}(\eta(\tau, x))-c_{0}\left(\eta_{n}(\tau, x)\right)\right|+\left|c_{0}\left(\eta_{n}(\tau, x)\right)-c_{n, 0}\left(\eta_{n}(\tau, x)\right)\right| d \tau .
$$

Form Requirement $2.63 \mathrm{a}$ ) we get a bound $|D c(x)| \leq M_{\lambda}$ since $x \in \Omega_{T_{0} \leq \lambda}$. A similar bound $\left|D c_{0}(x)\right| \leq M_{\lambda}^{\prime}$ must hold for the transformed transport field $c_{0}$. This in turn implies the existence of a Lipschitz constant $L_{\lambda}$ for $c_{0}$ which is valid on $\Omega_{T_{0} \leq \lambda}$. For $0 \leq t \leq a(x)$ all points $\eta(t, x)$ and $\eta_{n}(t, x)$ are located in $\Omega_{T_{0} \leq \lambda}$. We conclude that

$$
\left|\eta_{n}-\eta\right|(t, x) \leq \int_{0}^{t} L_{\lambda} \cdot\left|\eta_{n}-\eta\right|(\tau, x) d \tau+t\left\|c_{0}-c_{n, 0}\right\|_{L^{\infty}\left(\Omega_{T_{0} \leq \lambda}\right)},
$$

and apply Gronwall's lemma (see [32])

$$
\left|\eta_{n}-\eta\right|(t, x) \leq t \cdot e^{t \cdot L_{\lambda}} \cdot\left\|c_{0}-c_{n, 0}\right\|_{L^{\infty}\left(\Omega_{T_{0} \leq \lambda}\right)} \leq C_{\lambda} \cdot\left\|c_{0}-c_{n, 0}\right\|_{L^{\infty}\left(\Omega_{T_{0} \leq \lambda}\right)} \cdot
$$

Because of $t \leq a(x)=\min \left\{T_{0}(x), T_{n, 0}(x)\right\} \leq \lambda$ for $x \in \Omega_{T_{0} \leq \lambda}$, the constant is $C_{\lambda}=\lambda \cdot e^{\lambda \cdot L_{\lambda}}$. The sequence $c_{n, 0}$ converges uniformly $c_{0}$ on $\Omega_{T_{0} \leq \lambda}$ since $c_{n} \rightarrow c$ and $\nabla T_{n, 0} \rightarrow \nabla T_{0}$ do so too. Consequently, we can make the difference $\left|\eta_{n}-\eta\right|(t, x)$ arbitrarily small. Based on that result, we estimate the difference $\left|\eta_{n}\left(T_{n, 0}(x), x\right)-\eta\left(T_{0}(x), x\right)\right|$. For abbreviation we set $\tau=T_{0}(x)$ and $\tau_{n}=$ $T_{n, 0}(x)$. Estimate (3.27) is valid for $t \leq a(x)=\min \left\{\tau, \tau_{n}\right\}$ only. Hence we need to supplement: in the case of $\tau=\min \left\{\tau, \tau_{n}\right\}$ we proceed as

$$
\begin{aligned}
\left|\eta_{n}\left(\tau_{n}, x\right)-\eta(\tau, x)\right| & \leq\left|\eta_{n}\left(\tau_{n}, x\right)-\eta_{n}(\tau, x)\right|+\left|\eta_{n}(\tau, x)-\eta(\tau, x)\right| \\
& \leq\left\|-c_{n, 0} \circ \eta_{n}(., x)\right\|_{L^{\infty}\left(\left[\tau, \tau_{n}\right]\right)} \cdot\left|\tau-\tau_{n}\right|+C_{\lambda} \cdot\left\|c_{0}-c_{n, 0}\right\|_{L^{\infty}\left(\Omega_{T_{0} \leq \lambda}\right)} \\
& \leq \frac{\left\|T_{0}-T_{n, 0}\right\|_{L^{\infty}\left(\Omega_{T_{0} \leq \lambda}\right)}}{\beta \cdot \bar{m}}+C_{\lambda} \cdot\left\|c_{0}-c_{n, 0}\right\|_{L^{\infty}\left(\Omega_{T_{0} \leq \lambda}\right)} .
\end{aligned}
$$


In the case of $\tau_{n}=\min \left\{\tau, \tau_{n}\right\}$ we supplement $\eta\left(\tau_{n}, x\right)$ instead $\eta_{n}(\tau, x)$ and obtain the same result. Now, we have everything to conclude that $\left|\eta_{n}\left(T_{n, 0}(x), x\right)-\eta\left(T_{0}(x), x\right)\right| \rightarrow 0$. And in the case of continuous boundary data $u_{0}$, we infer from dominated convergence that $\| u_{1, n}$ $u_{1} \|_{L^{1}\left(\Omega_{T_{0} \leq \lambda}\right)} \leq \varepsilon / 6$ when $n$ is sufficiently large. In the case of general $B V$ boundary data we approximate $u_{0}$ by a sequence $\left(u_{0}^{k}\right)_{k \in \mathbb{N}}$ of smooth functions. Note that $u_{1, n}$ and $u_{1}$ solve the PDEproblems with $f_{n}=f=0$ and boundary data $u_{0}$. Let $u_{1, n}^{k}$ and $u_{1}^{k}$ be the solutions with zero right-hand side and boundary data $u_{0}^{k}$. Then, Theorem 3.8 tells us that the differences in both cases are $\left\|u_{1, n}-u_{1, n}^{k}\right\|_{L^{\infty}(\Omega)}=\left\|u_{1}-u_{1}^{k}\right\|_{L^{\infty}(\Omega)}=\left\|u_{0}-u_{0}^{k}\right\|_{L^{\infty}(\partial \Omega)}$. Now, we choose $k$ so large that $2\left\|u_{0}-u_{0}^{k}\right\|_{L^{\infty}(\partial \Omega)} \cdot \mathcal{L}^{2}(\Omega) \leq \varepsilon / 6$ and obtain

$$
\left\|u_{1, n}-u_{1}\right\|_{L^{1}\left(\Omega_{T_{0} \leq \lambda}\right)} \leq 2\left\|u_{0}-u_{0}^{k}\right\|_{L^{\infty}(\partial \Omega)} \cdot \mathcal{L}^{2}(\Omega)+\left\|u_{1, n}^{k}-u_{1}^{k}\right\|_{L^{1}\left(\Omega_{T_{0} \leq \lambda}\right)} \leq \frac{\varepsilon}{3}
$$

where $\left\|u_{1, n}^{k}-u_{1}^{k}\right\|_{L^{1}\left(\Omega_{T_{0} \leq \lambda}\right)}$ was treated as described above. This step completes the $\varepsilon / 3$-argument which proves the $L^{1}$-convergence of $u_{n}$ to $u$.

$B V$-weak ${ }^{*}$-convergence. By Lemma 3.5 all the functions $u, u_{n}$ are elements of $B V(\Omega)$. Using the uniform bounds $\left\|f_{n}\right\|_{\infty} \leq M,\left\|\nabla f_{n}\right\|_{\infty} \leq M_{1},\left\|D c_{n}\right\|_{L^{1}(\Omega)} \leq M_{2},\left\|D N_{n}\right\|_{L^{1}(\Omega)} \leq M_{3}, \mathcal{H}^{1}\left(\Sigma_{n}\right) \leq$ $M_{4}$, and $\bar{m} \leq m_{n, 0}$, we learn from (3.20) and (3.21) that the sequence of total variations $\left|D u_{n}\right|(\Omega)$ is also bounded. By [4, Proposition 3.13], the boundedness of the sequence $\left|D u_{n}\right|(\Omega)$ along with the $L^{1}$-convergence $u_{n} \rightarrow u$ imply the $B V$-weak ${ }^{*}$-convergence of $u_{n}$ to $u$.

We have seen that the linear problem (3.1) has a unique solution in $B V(\Omega)$ and that the solution depends continuously on the data. In other words, this problem is well-posed in $B V(\Omega)$. Certainly, one might ask what the point of using the space $B V(\Omega)$ is, because if the boundary data $u_{0}$ were $C^{1}$, one could solve in $C^{1}(\Omega \backslash \Sigma)$. The advantage of our approach is that we can close the gap: $u$ is defined on all of $\Omega$, not only $\Omega \backslash \Sigma$, and we have a description of what happens to $u$ on $\Sigma$; we know that the solution belongs to $B V(\Omega)$. This is very useful, since in $B V(\Omega)$ it is easy to obtain compact subsets (with respect to the weak* topology) and compactness will be crucial for the existence theory of the quasi-linear problem.

4. The Quasi-Linear Problem. In Problem 2.8 the transport field as well as the right-hand side depend non-locally on the function $u$ which we are looking for. The first goal of this section is to prove the existence of a solution of the quasi-linear Problem 2.8. The plan for doing so is to interpret a solution $u$ as a fixed-point of a certain map $U$. The map $U$ in turn is obtained as an application of the theory of the previous section: by fixing the functional argument of the coefficients by some $v \in L^{1}(\Omega)$ the linear theory will give us a unique solution $U[v]$ which depends on $v$. Now, we will show that $U$ is a well-behaved self-mapping such that Schauder's fixed-point theorem provides us with the existence of a fixed-point $u=U[u]$. Any fixed-point of $U$ is a solution to Problem 2.8 .

4.1. Formulation as a Fixed-Point Problem. In order to formulate a fixed-point problem we need to find the function space $\mathfrak{X}$ in which $U: \mathfrak{X} \rightarrow \mathfrak{X}$ will be a well-behaved self-mapping. Here, we define the subsets of $B V(\partial \Omega)$ and $\mathfrak{X} \subset B V(\Omega)$ with which we work.

Definition 4.1. Let $\Omega$ be a domain and $\Sigma$ a stop set according to Requirements 2.1 and 2.2. Let $M_{1}, M_{2}, M_{3}$ be the bounds on $D_{x} c[v], f[v], \nabla_{x} f[v]$ from Requirements 2.6 and 2.7.

a) We denote by

$$
\mathfrak{B}=\mathfrak{B}(\partial \Omega):=\left\{v \in B V(\partial \Omega):\|v\|_{L^{\infty}(\partial \Omega)} \leq M_{4},|D v| \leq M_{5}\right\}
$$

the set of boundary functions. ( $M_{4}, M_{5}$ are generic constants.) 
b) Let $M_{*} \in \mathbb{R}$ and $M_{* *} \in \mathbb{R}$ be given by

$$
\begin{aligned}
M_{*} & :=\left(M_{4}+\frac{M_{2}}{\beta \cdot m_{0}}\right) \cdot \mathcal{L}^{2}(\Omega) \\
M_{* *} & :=2 \cdot \frac{M_{*}}{\mathcal{L}^{2}(\Omega)} \cdot \mathcal{H}^{1}(\Sigma)+\frac{M_{5}}{\beta \cdot m_{0}}+\left(\frac{M_{2}}{\beta}+\frac{M_{3}}{\beta^{2} \cdot m_{0}}\right) \cdot \mathcal{L}^{2}(\Omega)+\frac{M_{2}}{\beta^{3} \cdot m_{0}^{2}} \cdot\left(M_{1}+\|D N\|_{L^{1}(\Omega)}\right)
\end{aligned}
$$

We set

$$
\mathfrak{X}=\mathfrak{X}(\Omega):=\left\{v \in B V(\Omega):\|v\|_{L^{1}(\Omega)} \leq M_{*},|D v|(\Omega) \leq M_{* *}\right\} .
$$

The corollary below justifies the change of our viewpoint: Problem 2.8 is in fact equivalent to a fixed-point problem.

COROLLARY 4.2. Let all of the data $\Omega, \Sigma, T, c, f$ and $u_{0}$ be as assumed in Problem 2.8. Then,

a) for fixed $v \in L^{1}(\Omega)$, the unique solution of the linear problem with transport field $c[v]$ and right-hand side $f[v]$ defines a map / operator of type $U: L^{1}(\Omega) \rightarrow B V(\Omega)$.

b) The solution operator $U$, after restriction to $\mathfrak{X}$, is a self-mapping of type $U: \mathfrak{X} \rightarrow \mathfrak{X}$.

Proof.

a) Let $v \in L^{1}(\Omega)$ be arbitrary but fixed then the linear theory guarantees the existence of a unique solution $U[v]$ which belongs to $B V(\Omega)$ according to Lemma 3.5. In other words, the map $U: L^{1}(\Omega) \rightarrow B V(\Omega)$ is well-defined.

b) Let $U[v]$ be the solution of the linear problem. Lemma 3.5 provides us with the following estimate of the $L^{\infty}$-norm

$$
\|U[v]\|_{L^{\infty}(\Omega)} \leq\left\|u_{0}\right\|_{L^{\infty}(\partial \Omega)}+\frac{\|f[v]\|_{\infty}}{\beta \cdot m_{0}}
$$

and with an estimate of the total variation

$$
\begin{aligned}
|D U[v]|(\Omega) \leq & 2 \cdot\|U[v]\|_{L^{\infty}(\Omega)} \cdot \mathcal{H}^{1}(\Sigma)+\frac{\left|D u_{0}\right|}{\beta \cdot m_{0}}+\left(\frac{\|f[v]\|_{\infty}}{\beta}+\frac{\|\nabla f[v]\|_{\infty}}{\beta^{2} \cdot m_{0}}\right) \cdot \mathcal{L}^{2}(\Omega) \\
& +\frac{\|f[v]\|_{\infty}}{\beta^{3} \cdot m_{0}^{2}} \cdot\left(\|D c[v]\|_{L^{1}(\Omega)}+\|D N\|_{L^{1}(\Omega)}\right) .
\end{aligned}
$$

Using the bounds $M_{1}, M_{2}, M_{3}$ from Requirement 2.6 and 2.7, and also the bounds $M_{4}, M_{5}$ on $u_{0} \in \mathfrak{B}$, it is easy to see that the upper bounds

$$
\|U[v]\|_{L^{1}(\Omega)} \leq M_{*}, \quad|D U[v]|(\Omega) \leq M_{* *}
$$

hold independently of $v$. Summarizing, the operator $U$ is in fact of type $U: L^{1}(\Omega) \rightarrow \mathfrak{X}$. Because of $\mathfrak{X} \subset B V(\Omega) \subset L^{1}(\Omega)$ we can restrict the domain of $U$ to $\mathfrak{X}$, and thus $U: \mathfrak{X} \rightarrow \mathfrak{X}$ is a well-defined self-mappings.

4.1.1. Existence of a Fixed-Point. The next step is to conclude the existence of fixed-points $u=U[u]$. The tool for achieving this objective is Schauder's fixed-point theorem (cf. [34], [19]) which states

Let $\mathcal{X}$ be a Banach space and let $\mathcal{M} \subset \mathcal{X}$ be a non-empty, convex, and compact subset. Let the map $F: \mathcal{M} \rightarrow \mathcal{M}$ be continuous. Then, $F$ has a fixed-point $x \in \mathcal{M}$, i.e., $x=F(x)$.

The next two lemmas show that $\mathfrak{X}$ and $U: \mathfrak{X} \rightarrow \mathfrak{X}$ satisfy the assumptions of Schauder's theorem.

LEMMA 4.3.

a) The set $\mathfrak{X}$ of Definition 4.1 is non-empty, convex, and sequentially compact in the $B V$-weak ${ }^{*}$ topology.

b) The map $U: \mathfrak{X} \rightarrow \mathfrak{X}$ is sequentially continuous in the $B V$-weak* topology. 
Proof.

a) The set $\mathfrak{X}$, by its definition, is convex and obviously non-empty. Because $\mathfrak{X}$ is $\|\cdot\|_{B V(\Omega)}$ bounded, the sequential compactness is a consequence of [4, Theorem 3.23].

b) Let $\left(v_{n}\right)_{n \in \mathbb{N}}$ be a sequence in $\mathfrak{X}$ which tends to $v \in \mathfrak{X}$ in the $B V$-weak topology. Then, we have in particular $\left\|v-v_{n}\right\|_{L^{1}(\Omega)} \rightarrow 0$. Now set $c_{n}:=c\left[v_{n}\right], c:=c[v], f_{n}:=f\left[v_{n}\right], f:=f[v]$, $u_{n}:=U\left[v_{n}\right], u:=U[v]$. By Requirement 2.6 and 2.7 all the assumptions of Theorem 3.9 a) and b) are satisfied, which tells us that $U\left[v_{n}\right]=u_{n} \stackrel{*}{\rightarrow} u=U[v]$.

In order to apply Schauder's theorem we use a result from [10], which characterizes the weak $^{*}$ convergence of sequences in the dual space $\mathcal{X}^{\prime}$ of a separable normed space $\mathcal{X}$.

LEMMA 4.4. Let $(\mathcal{X},\|\cdot\|)$ be a separable normed space. Let $\sigma=\left(\sigma_{n}\right)_{n \in \mathbb{N}}$ be a sequence with $\left\|\sigma_{n}\right\|=1$ and $\mathcal{X}=\overline{\operatorname{span} \sigma}$. Let $\left(\mathcal{X}^{\prime},\|.\|^{\prime}\right)$ be the dual space with induced norm. Then,

a) the function $\left\|x^{\prime}\right\|_{\sigma}:=\sum_{n=1}^{\infty} 2^{-n}\left|x^{\prime}\left(\sigma_{n}\right)\right|$ defines a norm on the dual space $\mathcal{X}^{\prime}$.

b) a $\|\cdot\|^{\prime}$-bounded sequence $\left(x_{k}^{\prime}\right)_{k \in \mathbb{N}}$ in the dual space $\mathcal{X}^{\prime}$ converges to $x^{\prime} \in \mathcal{X}^{\prime}$ in weak ${ }^{*}$-topology if and only if it converges with regard to $\|.\|_{\sigma}$, i.e., $\left\|x_{k}^{\prime}-x^{\prime}\right\|_{\sigma} \rightarrow 0$, as $k \rightarrow \infty$.

Proof. Part a) is [10, Lemma 1], while part b) is [10, Theorem 1].

Finally, we come to our central existence result.

THEOREM 4.5. The map $U: \mathfrak{X} \rightarrow \mathfrak{X}$ admits a fixed-point $u=U[u]$.

Proof. In [4, Remark 3.12] we learn that $B V(\Omega)=\mathcal{X}^{\prime}$ is the dual of a separable space $\mathcal{X}$. By Lemma 4.4 we can introduce a new norm $\|\cdot\|_{\sigma}$ which characterizes the weak ${ }^{*}$-topology on $B V(\Omega)$. In terms of the new norm we see that Lemma 4.3 part a) tells us that $\mathfrak{X}$ is non-empty, convex and $\|\cdot\|_{\sigma}$-compact, while part b) tells us that the operator $U: \mathfrak{X} \rightarrow \mathfrak{X}$ is $\|\cdot\|_{\sigma}$-continuous. Now, Schauder's fixed-point theorem yields the existence of a fixed-point $u=U[u]$.

4.1.2. Example of Non-Uniqueness. We consider an almost linear problem on $\Omega \backslash \Sigma$, where the transport field does not depend on $u$ and where the right-hand side does not depend on $x$ :

$$
\langle c(x), D u\rangle=f[u] \cdot \mathcal{L}^{2},\left.\quad u\right|_{\partial \Omega}=0, \quad f[u]:=g\left(\|u\|_{L^{1}(\Omega)}\right) .
$$

Here, $g: \mathbb{R} \rightarrow \mathbb{R}$ is a continuous bounded function so that $f$ satisfies Requirement 2.7. Having fixed the functional argument of $f$, the solution $U[v]$ is given according to (3.18) and reduces to

$$
U[v](x)=\int_{0}^{T_{0}(x)} f_{0}[v] \circ \eta(\tau, x) d \tau=f[v] \int_{0}^{T_{0}(x)} \frac{1}{\left\langle c, \nabla T_{0}\right\rangle} \circ \eta(\tau, x) d \tau=f[v] \int_{0}^{T_{0}(x)}\left|\eta^{\prime}(\tau, x)\right| d \tau .
$$

The last equality in (4.4) becomes obvious by looking at the characteristic ODE $\eta^{\prime}=-c_{0} \circ \eta$ : we see that $\left|\eta^{\prime}\right|=1 /\left\langle c, \nabla T_{0}\right\rangle \circ \eta$ since $|c|=1$. Let $a(x)$ denote the arc-length of the curve $\eta(., x)$ which connects $x=\eta(0, x)$ and the point $\eta\left(T_{0}(x), x\right)$ on the boundary $\partial \Omega$, then (4.4) can be written as $U[v](x)=f[v] \cdot a(x)=g\left(\|v\|_{L^{1}(\Omega)}\right) \cdot a(x)$. Thus, if $u=U[u]$ is a fixed-point, it must satisfy

$$
u(x)=g\left(\|u\|_{L^{1}(\Omega)}\right) \cdot a(x) .
$$

Consequently, $u(x)=\alpha \cdot a(x)$ is a scalar multiple of the arc-length function $a$. Substituting $u(x)=$ $\alpha \cdot a(x)$ in (4.5) accordingly yields a scalar fixed-point problem

$$
\alpha \cdot a(x)=g\left(\alpha \cdot\|a\|_{L^{1}(\Omega)}\right) \cdot a(x) \quad \Rightarrow \quad \alpha=g\left(\alpha \cdot\|a\|_{L^{1}(\Omega)}\right)=: \tilde{g}(\alpha) .
$$

If $\tilde{g}$ is a continuous bounded function with many fixed-points $\alpha$, e.g. $\tilde{g}(t)=\max \{0, \min \{t, 1\}\}$, then the operator $U$ has as many fixed-points as $\tilde{g}$. 
4.2. Uniqueness and Stability under Causality. In this section we will show the uniqueness of the fixed-point given that the functional dependence of the coefficients $c$ and $f$ is of Volterratype, i.e., if it is causal. The notion of time induced by the time function $T$ allows us to define causality.

DEFINITION 4.6. Let $\Omega$ be a domain and $T$ a time function according to Requirements 2.1 and 2.4. Let $\mathcal{F}(\Omega)$ and $\mathcal{G}(\Omega)$ be function spaces defined on $\Omega$ and let $f$ be an operator

$$
f: \mathcal{F}(\Omega) \rightarrow \mathcal{G}(\Omega), \text { with } \quad f[.](x): \mathcal{F}(\Omega) \rightarrow \mathbb{R}^{d}, \quad x \in \Omega .
$$

Let $T(x)$ be the time of the point $x \in \Omega$, then we call the lower level-set $\Omega_{T<T(x)}$ of $T$ the "past" of $x$. We say that the functional dependence of $f$ is causal or of Volterra-type if the equation

$$
f[v](x)=f\left[v \cdot \mathbb{1}_{\Omega_{T<T(x)}}\right](x)
$$

is valid, i.e., if the value $f[v](x)$ depends only on those values which $v$ takes on in the past of $x$.

This causality provides us with the following domain restriction feature: let $\lambda$ be in the range of $T$ and let $v \in \mathcal{F}(\Omega)$. For $x \in \Omega_{T<\lambda}$ we have the inclusion $\Omega_{T<T(x)} \subset \Omega_{T<\lambda}$ which implies

$$
f[v](x)=f\left[v \cdot \mathbb{1}_{\Omega_{T<T(x)}}\right](x)=f\left[v \cdot \mathbb{1}_{\Omega_{T<\lambda}}\right](x) .
$$

Hence, the domain restriction (onto $\Omega_{T<\lambda}$ ) $f: \mathcal{F}\left(\Omega_{T<\lambda}\right) \rightarrow \mathcal{G}\left(\Omega_{T<\lambda}\right)$ is well-defined.

The requirements on transport fields and right-hand sides are updated as follows. Regarding the causality statements, we refer to the same given time function $T$.

REQUIREMENT 4.7. Let $c: L^{1}(\Omega) \rightarrow C^{1}(\Omega \backslash \Sigma)^{2}$ be a transport field according to Requirement 2.6. $c$ is required to satisfy in addition:

a) the functional dependence of $c$ is of Volterra-type.

b) $c$ is Lipschitz in $v: \quad\|c[v]-c[w]\|_{\infty} \leq L_{1} \cdot\|v-w\|_{L^{1}(\Omega)}$.

REQUIREMENT 4.8. Let $f: L^{1}(\Omega) \rightarrow C^{1}(\bar{\Omega})$ be a right-hand side according to Requirement 2.7. $f$ is required to satisfy in addition:

a) the functional dependence of $f$ is of Volterra-type.

b) $f$ is Lipschitz in $v: \quad\|f[v]-f[w]\|_{\infty} \leq L_{2} \cdot\|v-w\|_{L^{1}(\Omega)}$.

Our first result is that $U$ inherits the causality of the coefficients.

LEMMA 4.9. If the functional dependence of the coefficients $c, f$ is causal, then the functional dependence of the solution operator $U: \mathfrak{X} \rightarrow \mathfrak{X}$ is causal too.

Proof. Let $v \in \mathfrak{X}$ be arbitrary but fixed. Let $T_{0}, c_{0}$, and $f_{0}$ denote the transformed versions of $T, c$, and $f$. Clearly, $c_{0}$ and $f_{0}$ are as causal as $c$ and $f$. Let $\eta[v](., x)$ the backward characteristic; $\eta[v](., x)$ is the solution of

$$
y^{\prime}=-c_{0}[v](y), \quad y(0)=x .
$$

For every $t \in] 0, T_{0}(x)\left[\right.$ we know that $\eta[v](t, x) \in \Omega_{T_{0}<T_{0}(x)}$. Thus, $\eta[v](., x)$ depends only on the restriction $-\left.c_{0}[v]\right|_{\Omega_{T_{0}<T_{0}(x)}}$ which depends only on $v \cdot \mathbb{1}_{\Omega_{T_{0}<T_{0}(x)}}$. In other words $\eta[v](., x)$ is causal. From the representation of $U[v](x)$

$$
U[v](x)=u_{0}\left(\eta[v]\left(T_{0}(x), x\right)\right)+\int_{0}^{T_{0}(x)} f_{0}[v] \circ \eta[v](\tau, x) d \tau,
$$

it is now obvious that $U[v](x)=U\left[v \cdot \mathbb{1}_{\Omega_{T_{0}<T_{0}(x)}}\right](x)$.

In the following we investigate fixed-points of $U: \mathfrak{X}_{\lambda} \rightarrow \mathfrak{X}_{\lambda}$, where $\mathfrak{X}_{\lambda}=\mathfrak{X}\left(\Omega_{T_{0}<\lambda}\right):=$ $\left\{\left.v\right|_{\Omega_{T_{0}<\lambda}}: v \in \mathfrak{X}\right\}$ denotes the domain-restricted version of $\mathfrak{X}$. By Lemma 4.9 the operator $U$ : $\mathfrak{X} \rightarrow \mathfrak{X}$ is causal, and so the domain-restricted version $U: \mathfrak{X}_{\lambda} \rightarrow \mathfrak{X}_{\lambda}$ is well-defined. The proof of Lemma 4.9 shows in addition that $U[v]$ (as function of $x$ ) solves the domain-restricted linear problem. Finally, every fixed-point $u$ of the original operator $U: \mathfrak{X} \rightarrow \mathfrak{X}$ after domain restriction $\left.u\right|_{\Omega_{T_{0}<\lambda}}$ belongs to $\mathfrak{X}_{\lambda}$ and is a fixed-point of $U: \mathfrak{X}_{\lambda} \rightarrow \mathfrak{X}_{\lambda}$. As the existence question has been settled in Theorem 4.5, the next step is to prove the uniqueness. 
4.2.1. Uniqueness of the Fixed-Point. In this section we will show that, for any choice of $0<\lambda<1$, the operator $U: \mathfrak{X}_{\lambda} \rightarrow \mathfrak{X}_{\lambda}$ is Lipschitz. Moreover, we will see that $U: \mathfrak{X}_{\lambda} \rightarrow \mathfrak{X}_{\lambda}$ is in fact contractive for a suitable choice of $\lambda$. The latter feature will then imply the uniqueness of the fixed-point.

For the estimation of the difference $U\left[v_{1}\right]-U\left[v_{2}\right]$ we prepare by setting up a PDE which is satisfied by the difference. For the purpose of abbreviation, we set $c_{i}:=c\left[v_{i}\right], f_{i}:=f\left[v_{i}\right]$, $i \in\{1,2\}$. Let $u_{1}$ and $u_{2}$ respectively denote the solutions of the two linear problems in $\Omega_{T_{0}<\lambda}$

$$
\begin{aligned}
& \left\langle c_{1}(x), D u_{1}\right\rangle=f_{1}(x) \cdot \mathcal{L}^{2} \quad \text { in } \Omega_{\lambda},\left.\quad u_{1}\right|_{\partial \Omega}=u_{0,1}, \\
& \text { and } \quad\left\langle c_{2}(x), D u_{2}\right\rangle=f_{2}(x) \cdot \mathcal{L}^{2} \quad \text { in } \Omega_{\lambda},\left.\quad u_{2}\right|_{\partial \Omega}=u_{0,2} \text {. }
\end{aligned}
$$

For the first considerations we use different boundary data. When setting $u_{0,1}=u_{0,2}=u_{0}$ later on, we will obtain the relations

$$
u_{1}=U\left[v_{1}\right], \quad u_{2}=U\left[v_{2}\right] .
$$

Let $w$ denote the difference $w:=u_{1}-u_{2}$. After having subtracted the problems from each other, the difference $w$ must satisfy the PDE

$$
\left\langle c_{1}(x), D w\right\rangle=\left(f_{1}(x)-f_{2}(x)\right) \cdot \mathcal{L}^{2}-\left\langle c_{1}(x)-c_{2}(x), D u_{2}\right\rangle \quad \text { in } \quad \Omega_{T_{0}<\lambda},
$$

and the boundary condition $\left.w\right|_{\partial \Omega}=w_{0}:=u_{0,1}-u_{0,2}$.

As in the proof of Theorem 3.8 we see that $w$ is the unique solution of this linear PDE. But, in order to solve for $w$, we cannot directly apply the method of characteristics, since the right-hand side is not an absolutely continuous measure. Instead we approximate the right-hand side by absolutely continuous measures. Since $u_{2} \in B V(\Omega)$, by [4, Theorem 3.9], there exists a sequence $\left(u_{2, n}\right)_{n \in \mathbb{N}}$ of $C^{\infty}(\Omega)$-functions which converges strictly to $u_{2}$, i.e.,

$$
\left\|u_{2}-u_{2, n}\right\|_{L^{1}(\Omega)} \rightarrow 0 \text { and } \quad|| D u_{2}|(\Omega)-| D u_{2, n}|(\Omega)| \rightarrow 0 .
$$

Moreover, we have $D u_{2, n}=\nabla u_{2, n}(x) \cdot \mathcal{L}^{2}$ and $\left|D u_{2, n}\right|(\Omega)=\left\|\nabla u_{2, n}\right\|_{L^{1}(\Omega)}$. Using such a sequence we obtain an approximate problem

$$
\left\langle c_{1}(x), D w_{n}\right\rangle=\left(f_{1}(x)-f_{2}(x)-\left\langle c_{1}(x)-c_{2}(x), \nabla u_{2, n}(x)\right\rangle\right) \cdot \mathcal{L}^{2} \quad \text { in } \quad \Omega_{T_{0}<\lambda},
$$

with a sequence of solutions $w_{n}$ which we can construct. We scale with $1 /\left\langle c_{1}, \nabla T_{0}\right\rangle$ and set

$$
c_{1,0}:=\frac{c_{1}}{\left\langle c_{1}, \nabla T_{0}\right\rangle}, \quad c_{2}^{0}:=\frac{c_{2}}{\left\langle c_{1}, \nabla T_{0}\right\rangle}, \quad f_{1,0}:=\frac{f_{1}}{\left\langle c_{1}, \nabla T_{0}\right\rangle}, \quad f_{2}^{0}:=\frac{f_{2}}{\left\langle c_{1}, \nabla T_{0}\right\rangle}
$$

(if we were to be consistent, we would set $f_{2,0}:=f_{2} /\left\langle c_{2}, \nabla T_{0}\right\rangle$, which differs from $f_{2}^{0}$ ). The family of forward characteristics $\xi(., s)$ of PDE (4.7) are trajectories of $c_{1,0}$ and we obtain $w_{n}$ in characteristic variables by

$$
w_{n} \circ \xi(t, s)=\gamma^{*} w_{0}(s)+\int_{0}^{t}\left(f_{1,0}-f_{2}^{0}-\left\langle c_{1,0}-c_{2}^{0}, \nabla u_{2, n}\right\rangle\right) \circ \xi(\tau, s) d \tau .
$$

The consideration of the sequence $w_{n}$ will not be of any use if $w_{n}$ does not tend to $w$ in an appropriate fashion. We will show the desired convergence in Lemma 4.11. But first, we rewrite $\left(w_{n}-w\right) \circ \xi$. Because the PDE for $u_{1}$ has the same transport field $c_{1}$, we have

$$
\begin{aligned}
& u_{1} \circ \xi(t, s)=\gamma^{*} u_{0,1}+\int_{0}^{t} f_{1,0} \circ \xi(\tau, s) d \tau, \quad \text { and } \\
& u_{2, n} \circ \xi(t, s)=u_{2, n} \circ \gamma(s)+\int_{0}^{t}\left\langle c_{1,0}, \nabla u_{2, n}\right\rangle \circ \xi(\tau, s) d \tau
\end{aligned}
$$


by the fundamental theorem of calculus. With the last two observations, and with $w_{0}=u_{0,1}-$ $u_{0,2}, w=u_{1}-u_{2}$, we end up with

$$
\left(w_{n}-w\right) \circ \xi=\left(u_{2}-u_{2, n}\right) \circ \xi+\left(\gamma^{*} u_{2, n}-\gamma^{*} u_{0,2}\right)+\int_{0}^{t}\left(\left\langle c_{2}^{0}, \nabla u_{2, n}\right\rangle-f_{2}^{0}\right) \circ \xi(\tau, .) d \tau .
$$

As a second step of preparation, we will show that there are uniform bounds on the determinant of $D \xi$.

LEMMA 4.10. For fixed $v \in L^{1}(\Omega)$, let $\left.\xi[v]:\right] 0,1[\times] a, b[\rightarrow \Omega \backslash(S \cup \Sigma)$ denote the diffeomorphism from Corollary 3.3 given by the solution of the IVP

$$
y^{\prime}=c_{0}[v](y) \quad y(0)=\gamma(s) .
$$

Then, for $0<\lambda<1$ there are bounds $k_{\lambda}$ and $K_{\lambda}$ such that

$$
\left.0<k_{\lambda} \leq \operatorname{det} D \xi[v](t, s) \leq K_{\lambda} \quad \forall(t, s) \in\right] 0, \lambda[\times] a, b[.
$$

The bounds $k_{\lambda}$ and $K_{\lambda}$ depend on $\lambda$, but not on $v$. Moreover, $k_{\lambda}$ decreases, while $K_{\lambda}$ increases monotonically with $\lambda$.

Proof. The right-hand side of the IVP is given by $c_{0}[v]=c[v] /\left\langle c[v], \nabla T_{0}\right\rangle$. By Requirement 2.6 part 3a), there is the uniform bound $\left|D_{x} c[v](x)\right| \leq M_{\varepsilon}, x \in \Omega \backslash V_{\varepsilon}$, and a similar bound $M_{0, \varepsilon}$ will hold for $D_{x} c_{0}[v](x)$. The diffeomorphism $\xi[v]$ maps the set $] 0, \lambda[\times] a, b\left[\right.$ onto $\Omega_{T_{0}<\lambda}$ for every $v$. Now, we choose $\varepsilon$ so small that $\Omega_{T_{0}<\lambda} \subset \Omega \backslash V_{\varepsilon}$ and obtain a bound that depends on $\lambda$ only:

$$
\left|D_{x} \mathcal{c}_{0}[v](x)\right| \leq M_{0, \varepsilon(\lambda)} \quad, \quad \forall x \in \Omega_{T_{0}<\lambda} \quad, \quad \forall v \in L^{1}(\Omega) .
$$

From Lemma 3.4 a) we know that $\operatorname{det} D \xi[v] \leq\left|\partial_{t} \xi[v]\right| \cdot\left|\partial_{s} \xi[v]\right| \leq\left|\partial_{s} \xi[v]\right| /\left(\beta \cdot m_{0}\right)$. Since $\partial_{s} \xi[v]$ solves the $\mathrm{ODE} \partial_{s} \xi[v]^{\prime}=D_{x} c_{0}[v] \circ \xi[v] \cdot \partial_{s} \xi[v]$ we estimate

$$
\left|\partial_{s} \xi[v]\right|(t, s) \leq\left\|\gamma^{\prime}\right\|_{\infty}+\int_{0}^{t} M_{0, \varepsilon(\lambda)}\left|\partial_{s} \xi[v]\right|(\tau, s) d \tau, \quad t \in[0, \lambda]
$$

An application of Gronwall's lemma gives $\left|\partial_{s} \xi[v]\right|(t, s) \leq\left\|\gamma^{\prime}\right\|_{\infty} \exp \left(\lambda \cdot M_{0, \varepsilon}(\lambda)\right)$ and leads to

$$
\left.\operatorname{det} D \xi[v](t, s) \leq \frac{\left\|\gamma^{\prime}\right\|_{\infty} \exp \left(\lambda \cdot M_{0, \varepsilon}(\lambda)\right)}{\beta \cdot m_{0}}=: K_{\lambda} \quad \forall(t, s) \in\right] 0, \lambda[\times] a, b[.
$$

For the lower bound consider the inverse $\xi[v]^{-1}(x)=\left(T_{0}(x), s[v](x)\right)$ on $\Omega_{T_{0}<\lambda}$, with $s[v](x)=$ $\gamma^{-1}\left(\eta[v]\left(T_{0}(x), x\right)\right)$ and the backward characteristics $\eta[v](., x)$. The determinant of $D_{x} \xi[v]^{-1}$ is bounded by det $D_{x} \xi[v]^{-1}(x) \leq\left|\nabla T_{0}(x)\right| \cdot\left|\nabla_{x} s[v](x)\right|$ and

$$
\nabla_{x} s[v](x)^{T}=\left(\gamma^{-1}\right)^{\prime}(\eta)^{T} \cdot\left(\partial_{t} \eta[v]\left(T_{0}(x), x\right) \cdot \nabla T_{0}(x)^{T}+\left.D_{x} \eta[v](t, x)\right|_{t=T_{0}(x)}\right) .
$$

Since $x \in \Omega_{T_{0}<\lambda}$, we can estimate $\left|D_{x} \eta[v](t, x)\right|$ in the same way as $\left|\partial_{s} \xi[v](t, s)\right|$ which gives us $\left|D_{x} \eta[v](t, x)\right| \leq \exp \left(\lambda \cdot M_{0, \varepsilon(\lambda)}\right)$. Finally, we observe that

$$
\operatorname{det} D_{x} \xi[v]^{-1}(x) \leq \frac{\left\|\nabla T_{0}\right\|_{L^{\infty}\left(\Omega_{\lambda}\right)}}{\min _{s \in[a, b]}\left|\gamma^{\prime}(s)\right|} \cdot\left(\frac{\left\|\nabla T_{0}\right\|_{L^{\infty}\left(\Omega_{\lambda}\right)}}{\beta \cdot m_{0}}+\exp \left(\lambda \cdot M_{0, \varepsilon(\lambda)}\right)\right), \quad x \in \Omega_{T_{0}<\lambda} .
$$

We set $1 / k_{\lambda}$ equal to the right-hand side of the last inequality. Then, we have

$$
\frac{1}{\operatorname{det} D \xi[v](t, s)}=\operatorname{det}\left((D \xi[v](t, s))^{-1}\right)=\left.\operatorname{det} D_{x} \xi[v]^{-1}(x)\right|_{x=\xi(t, s)} \leq \frac{1}{k_{\lambda}},
$$


for $(t, s) \in] 0, \lambda[\times] a, b\left[\right.$, since in this case $\xi(t, s) \in \Omega_{T_{0}<\lambda}$. Both bounds $k_{\lambda}$ and $K_{\lambda}$ do not depend on the choice of $v \in L^{1}(\Omega)$. The monotonicity properties of $k_{\lambda}$ and $K_{\lambda}$ as functions of $\lambda$ are obvious.

Now, we can turn to the approximation of $w$ by $w_{n}$.

LEMMA 4.11. Let $w$ and $w_{n}$ be as defined above. Interpret the $L^{1}\left(\Omega_{T_{0}<\lambda}\right)$-functions $w$ and $w_{n}$ as absolutely continuous measures $w(x) \cdot \mathcal{L}^{2}$ and $w_{n}(x) \cdot \mathcal{L}^{2}$ on $\Omega_{T_{0}<\lambda}$. Then, the sequence of measures $w_{n}(x) \cdot \mathcal{L}^{2}$ converges weakly* to $w(x) \cdot \mathcal{L}^{2}$.

Proof. Let $\varphi \in C_{0}\left(\Omega_{T_{0}<\lambda}\right)$ be a test function. By changing variables it follows that

$$
\int_{\Omega_{T_{0}<\lambda}}\left(w_{n}-w\right) \cdot \varphi d x=\int_{a}^{b} \int_{0}^{\lambda}\left(\left(w_{n}-w\right) \cdot \varphi\right) \circ \xi \cdot \operatorname{det} D \xi d t d s
$$

We use the representation of $\left(w_{n}-w\right) \circ \xi$ from (4.9) and study the convergence of the three summands in (4.9) separately. The first summand is estimated by

$$
\left|\int_{a}^{b} \int_{0}^{\lambda}\left(\left(u_{2, n}-u_{2}\right) \cdot \varphi\right) \circ \xi \cdot \operatorname{det} D \xi d t d s\right| \leq\left\|u_{2, n}-u_{2}\right\|_{L^{1}\left(\Omega_{T_{0}<\lambda}\right)}\|\varphi\|_{\infty},
$$

and the right-hand side tends to zero, because the sequence $u_{2, n}$ strictly tends to $u_{2}$ in $B V(\Omega)$. For the second summand we write

$$
\begin{aligned}
& \left|\int_{a}^{b} \int_{0}^{\lambda}\left(\gamma^{*} u_{2, n}(s)-\gamma^{*} u_{0,2}(s)\right) \cdot \varphi \circ \xi \cdot \operatorname{det} D \xi d t d s\right| \\
& =\left|\int_{a}^{b}\left(\gamma^{*} u_{2, n}(s)-\gamma^{*} u_{0,2}(s)\right) \cdot \int_{0}^{\lambda} \varphi \circ \xi \cdot \operatorname{det} D \xi d t d s\right| \leq C \cdot \int_{a}^{b}\left|\gamma^{*}\left(u_{2, n}-u_{0,2}\right)(s)\right| \cdot\left|\gamma^{\prime}(s)\right| d s .
\end{aligned}
$$

The constant $C$ comes from the estimate below. Let $k_{\lambda}$ and $K_{\lambda}$ be as in Lemma 4.10. With $k_{\lambda} \leq$ $\operatorname{det} D \xi(0, s) \leq\left|\gamma^{\prime}(s)\right| /\left(\beta \cdot m_{0}\right)$ we obtain

$$
\frac{\left|\int_{0}^{\lambda} \varphi \circ \xi(t, s) \cdot \operatorname{det} D \xi(t, s) d t\right|}{\left|\gamma^{\prime}(s)\right|} \leq \lambda \cdot \frac{K_{\lambda}}{\beta \cdot m_{0} \cdot k_{\lambda}} \cdot\|\varphi\|_{\infty}=: C .
$$

Moreover, we have

$$
\int_{a}^{b}\left|\gamma^{*}\left(u_{2, n}-u_{0,2}\right)(s)\right| \cdot\left|\gamma^{\prime}(s)\right| d s=\int_{\partial \Omega}\left|\left(u_{2, n}-u_{2}\right)\right|_{\partial \Omega}(x)\left|d \mathcal{H}^{1}(x)=\left\|\left.\left(u_{2, n}-u_{2}\right)\right|_{\partial \Omega}\right\|_{L^{1}\left(\partial \Omega, \mathcal{H}^{1}\right)} .\right.
$$

Here we apply the trace operator for $B V$-functions $.\left.\right|_{\partial \Omega}: B V(\Omega) \rightarrow L^{1}\left(\partial \Omega, \mathcal{H}^{1}\right),\left.v \rightarrow v\right|_{\partial \Omega}$. According to [4, Theorem 3.88] the trace operator is continuous in the strict topology on $B V(\Omega)$. Hence, $\left\|\left.\left(u_{2, n}-u_{2}\right)\right|_{\partial \Omega}\right\|_{L^{1}\left(\partial \Omega, \mathcal{H}^{1}\right)}$ tends to zero as $n$ tends to infinity.

Let $\psi(t, s):=\varphi \circ \xi(t, s) \cdot \operatorname{det} D \xi(t, s)$. By changing the order of integration, we get for the 
third summand

$$
\begin{aligned}
& \int_{a}^{b} \int_{0}^{\lambda} \int_{0}^{t}\left(\left\langle c_{2}^{0}, \nabla u_{2, n}\right\rangle-f_{2}^{0}\right) \circ \xi(\tau, s) d \tau \cdot \psi(t, s) d t d s \\
& =\int_{a}^{b} \int_{0}^{\lambda}\left(\left\langle c_{2}^{0}, \nabla u_{2, n}\right\rangle-f_{2}^{0}\right) \circ \xi(\tau, s)\left(\int_{\tau}^{\lambda} \psi(t, s) d t\right) d \tau d s \\
& =\int_{a}^{b} \int_{0}^{\lambda}\left(\left\langle c_{2}^{0}, \nabla u_{2, n}\right\rangle-f_{2}^{0}\right) \circ \xi \cdot h \circ \xi \cdot \operatorname{det} D \xi d \tau d s=\int_{\Omega_{T_{0}<\lambda}}\left(\left\langle c_{2}^{0}, \nabla u_{2, n}\right\rangle-f_{2}^{0}\right) \cdot h d x .
\end{aligned}
$$

In the second equality we have used the function $h$ which comes from the following consideration: by the definition of $\psi$ and since $\xi$ is a diffeomorphism, there is a continuous function $h \in C\left(\bar{\Omega}_{T_{0}<\lambda}\right)$ such that

$$
h \circ \xi(\tau, s)=(\operatorname{det} D \xi(\tau, s))^{-1} \cdot\left(\int_{\tau}^{\lambda} \psi(t, s) d t\right) .
$$

Next, we use the fact that $u_{2}$ solves the $\operatorname{PDE}\left\langle c_{2}^{0}(x), D u_{2}\right\rangle=f_{2}^{0}(x) \cdot \mathcal{L}^{2}$. Setting $\hat{\varphi}(x):=h(x) \cdot c_{2}^{0}(x)$ as a new test function which belongs to $C\left(\overline{\Omega_{T_{0}<\lambda}}\right)^{2}$ we have

$$
\int_{\Omega_{T_{0}<\lambda}}\left(\left\langle c_{2}^{0}, \nabla u_{2, n}\right\rangle-f_{2}^{0}\right) \cdot h d x=\int_{\Omega_{T_{0}<\lambda}}\left\langle\hat{\varphi}, \nabla u_{2, n}\right\rangle d x-\int_{\Omega_{T_{0}<\lambda}}\left\langle\hat{\varphi}, d D u_{2}(x)\right\rangle .
$$

Owing again to the strict convergence of $u_{2, n}$ to $u_{2}$, we conclude that the last integral expression tends to zero as $n \rightarrow \infty$. Summarizing the three steps above we obtain

$$
\int_{\Omega_{T_{0}<\lambda}}\left(w_{n}-w\right)(x) \cdot \varphi(x) d x \rightarrow 0 \quad \forall \varphi \in C_{0}\left(\Omega_{T_{0}<\lambda}\right),
$$

which means $w_{n}(x) \cdot \mathcal{L}^{2} \stackrel{*}{\rightarrow} w(x) \cdot \mathcal{L}^{2}$ on $\Omega_{T_{0}<\lambda}$.

Based on the properties of the sequence $w_{n}$ we show in the following that the operator $U$ is Lipschitz.

LEMMA 4.12. Let $\lambda, h \geq 0$ be such that $\lambda+h<1$. We set

$$
\Omega_{\lambda+h, \lambda}:=\Omega_{T_{0}<\lambda+h} \backslash \Omega_{T_{0}<\lambda}=\left\{z \in \Omega: \lambda \leq T_{0}(z)<\lambda+h\right\} .
$$

Then, the difference $w=u_{1}-u_{2}$ satisfies

$$
\begin{aligned}
\|w\|_{L^{1}\left(\Omega_{T_{0}<\lambda+h}\right)} \leq & (\lambda+h) \cdot C_{\lambda+h} \cdot\left\|u_{0,1}-u_{0,2}\right\|_{L^{1}\left(\partial \Omega, \mathcal{H}^{1}\right)} \\
& +C_{\lambda+h} \cdot \mathcal{L}^{2}(\Omega) \cdot\left((\lambda+h) \cdot\left\|f_{1}-f_{2}\right\|_{L^{\infty}\left(\Omega_{T_{0}<\lambda}\right)}+h \cdot\left\|f_{1}-f_{2}\right\|_{L^{\infty}\left(\Omega_{T_{0}<\lambda+h}\right)}\right) \\
& +C_{\lambda+h} \cdot M_{* *} \cdot\left((\lambda+h) \cdot\left\|c_{1}-c_{2}\right\|_{L^{\infty}\left(\Omega_{T_{0}<\lambda}\right)}+h \cdot\left\|c_{1}-c_{2}\right\|_{L^{\infty}\left(\Omega_{T_{0}<\lambda+h}\right)}\right) .
\end{aligned}
$$

Here, the factor $C_{\lambda}:=K_{\lambda} /\left(\beta \cdot m_{0} \cdot k_{\lambda}\right)$ is an increasing function of $\lambda$.

Proof. We use the approximation of $w$ by $w_{n}$ again. Because of the weak*-convergence according to Lemma 4.11 and because of the lower semi-continuity of the total variation with respect to the weak*-convergence (for the semi-continuity of norms, e.g., see [2]), we have

$$
\|w\|_{L^{1}\left(\Omega_{T_{0}<\lambda}\right)}=\left|w \cdot \mathcal{L}^{2}\right|\left(\Omega_{T_{0}<\lambda}\right) \leq \liminf _{n \rightarrow \infty}\left|w_{n} \cdot \mathcal{L}^{2}\right|\left(\Omega_{T_{0}<\lambda}\right)=\liminf _{n \rightarrow \infty}\left\|w_{n}\right\|_{L^{1}\left(\Omega_{T_{0}<\lambda}\right)} .
$$


Thus, we can estimate $\left\|w_{n}\right\|_{L^{1}\left(\Omega_{T_{0}<\lambda}\right)}$ instead. Using the representation of $w_{n}$ by (4.8), we obtain

$$
\begin{aligned}
& \left\|w_{n}\right\|_{L^{1}\left(\Omega_{T_{0}<\lambda+h}\right)}=\int_{a}^{b} \int_{0}^{\lambda+h}\left|w_{n}\right| \circ \xi \cdot \operatorname{det} D \xi d t d s \leq \int_{a}^{b} \int_{0}^{\lambda+h}\left|\gamma^{*}\left(u_{0,1}-u_{0,2}\right)\right| \operatorname{det} D \xi d t d s \\
& +\int_{a}^{b} \int_{0}^{\lambda+h}\left(\int_{0}^{t}\left|f_{1,0}-f_{2}^{0}\right| \circ \xi(\tau, s)+\left|\left\langle\left(c_{1,0}-c_{2}^{0}\right), \nabla u_{2, n}\right\rangle\right| \circ \xi(\tau, s) d \tau\right) \operatorname{det} D \xi d t d s .
\end{aligned}
$$

By arguing the same way as in the proof of Lemma 4.11, we get for the first summand in the right-hand side of (4.11)

$$
\int_{a}^{b} \int_{0}^{\lambda+h}\left|\gamma^{*}\left(u_{0,1}-u_{0,2}\right)(s)\right| \cdot \operatorname{det} D \xi d t d s \leq(\lambda+h) \cdot C_{\lambda+h} \cdot\left\|u_{0,1}-u_{0,2}\right\|_{L^{1}\left(\partial \Omega, \mathcal{H}^{1}\right)} \cdot
$$

Regarding the third summand, let $g(\tau, s, t):=\left|c_{1,0}-c_{2}^{0}\right| \circ \xi(\tau, s) \cdot\left|\nabla u_{2, n}\right| \circ \xi(\tau, s) \cdot \operatorname{det} D \xi(t, s)$. Then, we estimate

$$
\begin{gathered}
\int_{a}^{b} \int_{0}^{\lambda+h} \int_{0}^{t}\left|\left\langle\left(c_{1,0}-c_{2}^{0}\right), \nabla u_{2, n}\right\rangle\right| \circ \xi(\tau, s) d \tau \cdot \operatorname{det} D \xi d t d s \leq \int_{a}^{b} \int_{0}^{\lambda+h} \int_{0}^{t} g(\tau, s, t) d \tau d t d s \\
\quad=\int_{a}^{b} \int_{0}^{\lambda+h}\left(\int_{\tau}^{\lambda+h} g d t\right) d \tau d s \leq \int_{a}^{b} \int_{0}^{\lambda}\left(\int_{0}^{\lambda+h} g d t\right) d \tau d s+\int_{a}^{\lambda+h} \int_{\lambda}^{\lambda+h}\left(\int_{\lambda}^{\lambda} g d t\right) d \tau d s .
\end{gathered}
$$

For the inner integrals with respect to $t$, we have

$$
\int_{\tau}^{\lambda} g d t=\left(\left|c_{1,0}-c_{2}^{0}\right| \cdot\left|\nabla u_{2, n}\right|\right) \circ \xi \int_{\tau}^{\lambda} \operatorname{det} D \xi(t, s) d t \leq\left(\left|c_{1,0}-c_{2}^{0}\right| \cdot\left|\nabla u_{2, n}\right|\right) \circ \xi \cdot(\lambda-\tau) \cdot K_{\lambda} .
$$

In the next step we multiply with $\operatorname{det} D \xi(\tau, s) / k_{\lambda}>1$ and take away the scaling factor, which is in the transport fields and the right-hand sides of the PDE, by $1 /\left\langle c_{1}, \nabla T_{0}\right\rangle \leq 1 /\left(m_{0} \cdot \beta\right)$ :

$$
\int_{\tau}^{\lambda} g d t \leq\left(\left|c_{1}-c_{2}\right| \cdot\left|\nabla u_{2, n}\right|\right) \circ \xi(\tau, s) \cdot \operatorname{det} D \xi(\tau, s) \cdot(\lambda-\tau) \cdot \frac{K_{\lambda}}{m_{0} \cdot \beta \cdot k_{\lambda}} .
$$

By the last result and the definition of $C_{\lambda}$ we infer on the one hand that

$$
\int_{a}^{b} \int_{0}^{\lambda} \int_{0}^{\lambda+h} g(\tau, s, t) d t d \tau d s \leq(\lambda+h) \cdot C_{\lambda+h}\left\|c_{1}-c_{2}\right\|_{L^{\infty}\left(\Omega_{T_{0}<\lambda}\right)} \cdot\left\|\nabla u_{2, n}\right\|_{L^{1}\left(T_{0}<\Omega_{\lambda}\right)},
$$

and on the other hand that

$$
\int_{a}^{b} \int_{\lambda}^{\lambda+h} \int_{\lambda}^{\lambda+h} g(\tau, s, t) d t d \tau d s \leq h \cdot C_{\lambda+h}\left\|c_{1}-c_{2}\right\|_{L^{\infty}\left(\Omega_{T_{0}<\lambda+h}\right)} \cdot\left\|\nabla u_{2, h}\right\|_{L^{1}\left(\Omega_{T_{0}<\lambda+h}\right)} .
$$

Finally, for the second summand in the right-hand side of (4.11) we need to perform the same steps with $g(\tau, s, t):=\left|f_{1,0}-f_{2}^{0}\right| \circ \xi(\tau, s) \cdot \mathbb{1}_{\Omega_{T_{0}<\lambda}} \circ \xi(\tau, s) \cdot \operatorname{det} D \xi(t, s)$. Up to some replacements we obtain the same estimate: $\left\|c_{1}-c_{2}\right\|_{L^{\infty}\left(\Omega_{T_{0}<\lambda}\right)}$ has to be replaced with $\left\|f_{1}-f_{2}\right\|_{L^{\infty}\left(\Omega_{T_{0}<\lambda}\right)}$ and 
$\left\|\nabla u_{2, n}\right\|_{L^{1}\left(\Omega_{T_{0}<\lambda}\right)}$ has to be replaced with $\mathcal{L}^{2}\left(\Omega_{T_{0}<\lambda}\right)$. Combining the last considerations we conclude the following estimate of $\left\|w_{n}\right\|_{L^{1}\left(\Omega_{T_{0}<\lambda+h}\right)}$

$$
\begin{aligned}
& \left\|w_{n}\right\|_{L^{1}\left(\Omega_{T_{0}<\lambda+h}\right)} \leq(\lambda+h) C_{\lambda+h} \cdot\left\|u_{0,1}-u_{0,2}\right\|_{L^{1}\left(\partial \Omega, \mathcal{H}^{1}\right)} \\
& +C_{\lambda+h} \mathcal{L}^{2}\left(\Omega_{T_{0}<\lambda+h}\right)\left((\lambda+h) \cdot\left\|f_{1}-f_{2}\right\|_{L^{\infty}\left(\Omega_{T_{0}<\lambda}\right)}+h \cdot\left\|f_{1}-f_{2}\right\|_{L^{\infty}\left(\Omega_{T_{0}<\lambda+h}\right)}\right) \\
& +C_{\lambda+h}\left\|\nabla u_{2, n}\right\|_{L^{1}\left(\Omega_{T_{0}<\lambda+h}\right)}\left((\lambda+h) \cdot\left\|c_{1}-c_{2}\right\|_{L^{\infty}\left(\Omega_{T_{0}<\lambda}\right)}+h \cdot\left\|c_{1}-c_{2}\right\|_{L^{\infty}\left(\Omega_{T_{0}<\lambda+h}\right)}\right) .
\end{aligned}
$$

Because of the strict convergence of $u_{2, n}$, we have $\left\|\nabla u_{2, n}\right\|_{L^{1}\left(\Omega_{T_{0}<\lambda+h}\right)} \rightarrow\left|D u_{2}\right|\left(\Omega_{T_{0}<\lambda+h}\right)$. Hence, taking the lower limit on both sides of (4.12) and plugging in the estimates $\left|D u_{2}\right|\left(\Omega_{T_{0}<\lambda+h}\right) \leq$ $M_{* *}$ and $\mathcal{L}^{2}\left(\Omega_{T_{0}<\lambda+h}\right) \leq \mathcal{L}^{2}(\Omega)$ finally yields (4.10). Lemma 4.10 tells us that $K_{\lambda}$ increases while $k_{\lambda}$ decreases with $\lambda$, hence $C_{\lambda}$ increases with $\lambda$.

COROLLARY 4.13. For any choice of $0<\lambda<1$ the operator $U: \mathfrak{X}_{\lambda} \rightarrow \mathfrak{X}_{\lambda}$ is $L^{1}$-Lipschitz

$$
\left\|U\left[v_{1}\right]-U\left[v_{2}\right]\right\|_{L^{1}\left(\Omega_{T_{0}<\lambda}\right)} \leq \lambda \cdot \kappa_{\lambda} \cdot\left\|v_{1}-v_{2}\right\|_{L^{1}\left(\Omega_{T_{0}<\lambda}\right)} .
$$

Here, $\kappa_{\lambda}$ is defined by $\kappa_{\lambda}:=C_{\lambda} \cdot\left(L_{2} \cdot \mathcal{L}^{2}(\Omega)+L_{1} \cdot M_{* *}\right)$ and is an increasing function of $\lambda$.

Proof. Let $v_{1}, v_{2} \in \mathfrak{X}_{\lambda}$. When we consider the operator $U$, we always use the same boundary data $u_{0} \in \mathfrak{B}$. Hence, (4.6) holds and we have $w=u_{1}-u_{2}=U\left[v_{1}\right]-U\left[v_{2}\right]$, since $u_{0,1}=u_{0,2}=u_{0}$. By using Lemma 4.12 with $h=0$ we see that

$$
\left\|U\left[v_{1}\right]-U\left[v_{2}\right]\right\|_{L^{1}\left(\Omega_{T_{0}<\lambda}\right)} \leq \lambda \cdot C_{\lambda} \cdot\left(\mathcal{L}^{2}(\Omega) \cdot\left\|f_{1}-f_{2}\right\|_{L^{\infty}\left(\Omega_{T_{0}<\lambda}\right)}+M_{* *} \cdot\left\|c_{1}-c_{2}\right\|_{L^{\infty}\left(\Omega_{T_{0}<\lambda}\right)}\right) .
$$

For the differences $f_{1}-f_{2}=f\left[v_{1}\right]-f\left[v_{2}\right]$ and $c_{1}-c_{2}=c\left[v_{1}\right]-c\left[v_{2}\right]$ we use the causality and the Lipschitz conditions, which we require. That is

$$
\begin{aligned}
\left\|f_{1}-f_{2}\right\|_{L^{\infty}\left(\Omega_{T_{0}<\lambda}\right)} & \leq\left\|f\left[v_{1} \cdot \mathbb{1}_{\Omega_{T_{0}<\lambda}}\right]-f\left[v_{2} \cdot \mathbb{1}_{\Omega_{T_{0}<\lambda}}\right]\right\|_{\infty} \\
& \leq L_{2} \cdot\left\|\left(v_{1}-v_{2}\right) \cdot \mathbb{1}_{\Omega_{T_{0}<\lambda}}\right\|_{L^{1}(\Omega)}=L_{2} \cdot\left\|v_{1}-v_{2}\right\|_{L^{1}\left(\Omega_{T_{0}<\lambda}\right)}
\end{aligned}
$$

and analogously $\left\|c_{1}-c_{2}\right\|_{L^{\infty}\left(\Omega_{T_{0}<\lambda}\right)} \leq L_{1} \cdot\left\|v_{1}-v_{2}\right\|_{L^{1}\left(\Omega_{T_{0}<\lambda}\right)}$. Now, (4.13) follows easily. Finally, $\kappa_{\lambda}$ increases with $\lambda$ since $C_{\lambda}$ does so too.

Now that we have brought together all ingredients we are able to show the uniqueness of the fixed-point.

THEOREM 4.14. Let the transport field $c: L^{1}(\Omega) \rightarrow C^{1}(\Omega \backslash \Sigma)^{2}$ and the right-hand side $f$ : $L^{1}(\Omega) \rightarrow C^{1}(\bar{\Omega})$ satisfy Requirements 4.7 and 4.8. Then, the solution operator $U: \mathfrak{X} \rightarrow \mathfrak{X}$ of the (non-restricted) original problem has a unique fixed-point $u \in \mathfrak{X}, u=U[u]$.

Proof. First, we show that, for any choice of $0<\lambda<1$, the domain-restricted operator $U: \mathfrak{X}_{\lambda} \rightarrow \mathfrak{X}_{\lambda}$ has a unique fixed-point. In order to do so we decompose $\Omega_{T_{0}<\lambda}$ into finitely many stripes $\Omega_{(l+1) h, l h}$ of "thickness" $h$. Let the step size $h$ be such that $h<1 / \kappa_{\lambda}$ and let $L:=$ $\lfloor\lambda / h\rfloor \in \mathbb{N}$ be the number of steps. Then, $\Omega_{T_{0}<\lambda}=\Omega_{h, 0} \cup \ldots \cup \Omega_{L h,(L-1) h} \cup \Omega_{\lambda, L h}$. For the first step, consider the operator $U: \mathfrak{X}_{h} \rightarrow \mathfrak{X}_{h}$ on $\Omega_{T_{0}<h}=\Omega_{h, 0}$. By Corollary 4.13 and the choice of $h$ we have a contraction

$$
\left\|U\left[v_{1}\right]-U\left[v_{2}\right]\right\|_{L^{1}\left(\Omega_{T_{0}<h}\right)} \leq h \cdot \kappa_{\lambda} \cdot\left\|v_{1}-v_{2}\right\|_{L^{1}\left(\Omega_{T_{0}<h}\right)}<\left\|v_{1}-v_{2}\right\|_{L^{1}\left(\Omega_{T_{0}<h}\right)} .
$$

If now $u_{1}=U\left[u_{1}\right]$ and $u_{2}=U\left[u_{2}\right]$ were two different fixed-points, we had, after domainrestriction onto $\Omega_{T_{0}<h}$, the nonsensical inequality

$$
\left\|u_{1}-u_{2}\right\|_{L^{1}\left(\Omega_{T_{0}<h}\right)}<\left\|u_{1}-u_{2}\right\|_{L^{1}\left(\Omega_{T_{0}<h}\right)},
$$

Consequently, all fixed-points coincide on the stripe $\Omega_{T_{0}<h}$. 
Next, we perform an inductive step. Assume that all fixed-points coincide on $\Omega_{l h}$, we show that they must also coincide on $\Omega_{(l+1) h}=\Omega_{l h+h}$. Let $u_{1}=U\left[u_{1}\right]$ and $u_{2}=U\left[u_{2}\right]$ be two different fixed-points again. We apply Lemma 4.12 with $\lambda=l h$ to $w=u_{1}-u_{2}$. Let $A:=\Omega_{T_{0}<(l+1) h}$. Because $u_{1}$ and $u_{2}$ coincide on $\Omega_{l h},(4.10)$ reduces to

$$
\|w\|_{L^{1}(A)} \leq C_{(l+1) h} \cdot h \cdot\left(\mathcal{L}^{2}(\Omega) \cdot\left\|f\left[u_{1}\right]-f\left[u_{2}\right]\right\|_{L^{\infty}(A)}+M_{* *} \cdot\left\|c\left[u_{1}\right]-c\left[u_{2}\right]\right\|_{L^{\infty}(A)}\right) .
$$

By employing the Lipschitz conditions on $c$ and $f$, and bearing in mind that $u_{1}$ and $u_{2}$ coincide on $\Omega_{l h}$, we obtain

$$
\left\|u_{1}-u_{2}\right\|_{L^{1}\left(\Omega_{(l+1) h, l h}\right)} \leq h \kappa_{(l+1) h} \cdot\left\|u_{1}-u_{2}\right\|_{L^{1}\left(\Omega_{(l+1) h, l h}\right)} .
$$

By the contractivity, $h \kappa_{(l+1) h} \leq h \kappa_{\lambda}<1$, we see that $\left\|u_{1}-u_{2}\right\|_{L^{1}\left(\Omega_{(l+1) h, l h}\right)}=0$ and so the fixedpoints also coincide on the next stripe $\Omega_{(l+1) h, l h}$. For the remaining last stripe we have to adapt the step size to $\hat{h}=\lambda-L h \leq h$ and the same argumentation applies.

As claimed, the domain-restricted operator $U: \mathfrak{X}_{\lambda} \rightarrow \mathfrak{X}_{\lambda}$ has a unique fixed-point for any choice of $0<\lambda<1$. Now the last step: assume by contradiction that the non-restricted operator $U: \mathfrak{X} \rightarrow \mathfrak{X}$ has two different fixed-points, $u_{1}$ and $u_{2}$. Therefor, $u_{1}$ and $u_{2}$ must differ on a subset $W \subset \Omega$ with $\mathcal{L}^{2}(W) \neq 0$. Because the stop set $\Sigma$ has Lebesgue measure zero, $\mathcal{L}^{2}(\Sigma)=0$, we can choose $0<\lambda<1$ so close to 1 that $\mathcal{L}^{2}\left(W \cap \Omega_{T_{0}<\lambda}\right) \neq 0$. Thus, we have

$$
\left\|u_{1}-u_{2}\right\|_{L^{1}\left(\Omega_{T_{0}<\lambda}\right)}=\left\|u_{1}-u_{2}\right\|_{L^{1}\left(W \cap \Omega_{T_{0}<\lambda}\right)} \neq 0 .
$$

But, because $\left.u_{1}\right|_{\Omega_{T_{0}<\lambda}}$ and $\left.u_{2}\right|_{\Omega_{T_{0}<\lambda}}$ are fixed-points of the domain-restricted operator $U: \mathfrak{X}_{\lambda} \rightarrow$ $\mathfrak{X}_{\lambda}$, we also have $\left\|u_{1}-u_{2}\right\|_{L^{1}\left(\Omega_{T_{0}<\lambda}\right)}=0$ by the previous uniqueness proof. A contradiction.

4.2.2. Continuous Dependence of the Fixed-Point. In this section, we show that the unique fixed-point depends $L^{1}$-continuously on the transport field, the right-hand side, and the boundary data. To this end we consider two linear problems:

$$
\begin{aligned}
& \langle c[v](x), D u\rangle=f[v](x) \cdot \mathcal{L}^{2} \quad \text { in } \quad \Omega \backslash \Sigma,\left.\quad u\right|_{\partial \Omega}=u_{0}, \\
& \langle\tilde{c}[\tilde{v}](x), D \tilde{u}\rangle=\tilde{f}[\tilde{v}](x) \cdot \mathcal{L}^{2} \quad \text { in } \quad \Omega \backslash \Sigma,\left.\quad \tilde{u}\right|_{\partial \Omega}=\tilde{u}_{0},
\end{aligned}
$$

where we assume that for both problems the same domain $\Omega$, the same stop set $\Sigma$, and the same time function $T$ (with transformed version $T_{0}$ ) are specified.

Moreover, we assume that $c$ and $\tilde{c}$ both satisfy the Requirements 2.6 and 4.7 with the same bounds, and also that $f$ and $\tilde{f}$ both satisfy the Requirements 2.7 and 4.8 with the same bounds. Finally, we assume $u_{0} \in \mathfrak{B}$ and $\tilde{u}_{0} \in \mathfrak{B}$. By the latter assumptions we are sure that we obtain two solution operators

$$
U: \mathfrak{X} \rightarrow \mathfrak{X}, \quad v \rightarrow U[v], \quad \text { and } \quad \tilde{U}: \mathfrak{X} \rightarrow \mathfrak{X}, \quad \tilde{v} \rightarrow \tilde{U}[\tilde{v}],
$$

which respectively correspond to the two linear problems above and possess the same domain and range $\mathfrak{X}$, which depends on all those bounds. In the following we view $\tilde{c}$ and $\tilde{f}$ as perturbed versions of $c$ and $f$. In order to measure perturbations we introduce the following norm:

DEFINITION 4.15. For maps of type $g: L^{1}(\Omega) \rightarrow C_{b}(\Omega \backslash \Sigma)^{d}$ or of type $g: L^{1}(\Omega) \rightarrow C(\bar{\Omega})^{d}$, $d \in \mathbb{N}$, we define the norm

$$
\|g\|_{0}:=\sup _{v \in L^{1}(\Omega)}\|g[v]\|_{\infty}
$$

THEOREM 4.16. Consider the two solution operators $U$ and $\tilde{U}$ as described above. Let $u$ and $\tilde{u}$ be the unique fixed-points of these operators, i.e., $u=U[u]$ and $\tilde{u}=\tilde{U}[\tilde{u}]$. Then, for every $\varepsilon>0$, one can find $\delta>0$ such that

$\|u-\tilde{u}\|_{L^{1}(\Omega)} \leq \varepsilon, \quad$ whenever $\left(\left\|u_{0}-\tilde{u}_{0}\right\|_{L^{1}\left(\partial \Omega, \mathcal{H}^{1}\right)}+\mathcal{L}^{2}(\Omega) \cdot\|f-\tilde{f}\|_{0}+M_{* *} \cdot\|c-\tilde{c}\|_{0}\right) \leq \delta$. 
Proof. Let $v, \tilde{v} \in \mathfrak{X}$ be arbitrary but fixed and set $u_{1}:=U[v], u_{2}:=\tilde{U}[\tilde{v}]$. On the restricted domain $\Omega_{T_{0}<\lambda}, 0<\lambda<1$, the difference $w:=u_{1}-u_{2}$ must solve the problem

$$
\langle c[v](x), D w\rangle=(f[v]-\tilde{f}[\tilde{v}])(x) \cdot \mathcal{L}^{2}-\left\langle(c[v]-\tilde{c}[\tilde{v}])(x), D u_{2}\right\rangle,\left.\quad w\right|_{\partial \Omega}=u_{0}-\tilde{u}_{0} .
$$

Again, we choose a sequence $u_{2, n} \in C^{\infty}(\Omega)$, strictly converging to $u_{2}$ in $B V(\Omega)$, to set up an approximate PDE which has $\nabla u_{2, n}(x) \cdot \mathcal{L}^{2}$ instead of $D u_{2}$. And again, the sequence $w_{n}$ of solutions converges weakly* to $w$. In order to proceed as in Lemma 4.12 we rewrite the right-hand side of the PDE as

$$
\begin{aligned}
\left\langle c[v](x), D w_{n}\right\rangle= & \left((f[v]-f[\tilde{v}])-\left\langle c[v]-c[\tilde{v}], \nabla u_{2, n}\right\rangle\right)(x) \cdot \mathcal{L}^{2} \\
& +\left((f[\tilde{v}]-\tilde{f}[\tilde{v}])-\left\langle c[\tilde{v}]-\tilde{c}[\tilde{v}], \nabla u_{2, n}\right\rangle\right)(x) \cdot \mathcal{L}^{2} .
\end{aligned}
$$

For the first summand of the new right-hand side we will apply the steps from the proof of Lemma 4.12. Regarding the second summand let $\xi=\xi[v]$ be the characteristics corresponding to the field $c[v]_{0}$ and let

$$
g(x)=\frac{\left|(f[\tilde{v}]-\tilde{f}[\tilde{v}])-\left\langle c[\tilde{v}]-\tilde{c}[\tilde{v}], \nabla u_{2, n}\right\rangle\right|}{\left\langle c[v], \nabla T_{0}\right\rangle}(x) .
$$

As in the proof of Lemma 4.12 we have to estimate the integral of $g$. After having changed the order of integration and having estimated the determinant, we obtain

$$
\begin{aligned}
& \int_{a}^{b} \int_{0}^{\lambda+h} \int_{0}^{t} g \circ \xi(\tau, s) d \tau \operatorname{det} D \xi d t d s \leq(\lambda+h) \cdot \frac{K_{\lambda+h}}{k_{\lambda+h}} \cdot \int_{a}^{b} \int_{0}^{\lambda+h} g \circ \xi(\tau, s) \operatorname{det} D \xi(\tau, s) d \tau d s \\
& \quad \leq(\lambda+h) \cdot C_{\lambda+h} \cdot \int_{\Omega_{\lambda+h}}\left|(f[\tilde{v}]-\tilde{f}[\tilde{v}])-\left\langle c[\tilde{v}]-\tilde{c}[\tilde{v}], \nabla u_{2, n}\right\rangle\right|(x) d x \\
& \quad \leq(\lambda+h) \cdot C_{\lambda+h}\left(\mathcal{L}^{2}(\Omega) \cdot\|f[\tilde{v}]-\tilde{f}[\tilde{v}]\|_{\infty}+\left\|\nabla u_{2, n}\right\|_{L^{1}(\Omega)} \cdot\|c[\tilde{v}]-\tilde{c}[\tilde{v}]\|_{\infty}\right) \\
& \quad \leq(\lambda+h) \cdot C_{\lambda+h}\left(\mathcal{L}^{2}(\Omega) \cdot\|f-\tilde{f}\|_{0}+\left\|\nabla u_{2, n}\right\|_{L^{1}(\Omega)} \cdot\|c-\tilde{c}\|_{0}\right) .
\end{aligned}
$$

Putting both estimates together and taking the lower limit, we end up with

$$
\begin{aligned}
\|w\|_{L^{1}\left(\Omega_{T_{0}<\lambda+h}\right)} & (\lambda+h) \cdot C_{\lambda+h} \cdot\left\|u_{0}-\tilde{u}_{0}\right\|_{L^{1}\left(\partial \Omega, \mathcal{H}^{1}\right)} \\
& +C_{\lambda+h} \cdot \mathcal{L}^{2}(\Omega)\left((\lambda+h) \cdot\|f[v]-f[\tilde{v}]\|_{L^{\infty}\left(\Omega_{T_{0}<\lambda}\right)}+h \cdot\|f[v]-f[\tilde{v}]\|_{L^{\infty}\left(\Omega_{T_{0}<\lambda+h}\right)}\right) \\
& +C_{\lambda+h} \cdot M_{* *}\left((\lambda+h) \cdot\|c[v]-c[\tilde{v}]\|_{L^{\infty}\left(\Omega_{T_{0}<\lambda}\right)}+h \cdot\|c[v]-c[\tilde{v}]\|_{L^{\infty}\left(\Omega_{T_{0}<\lambda+h}\right)}\right) \\
& +(\lambda+h) \cdot C_{\lambda+h}\left(\mathcal{L}^{2}(\Omega) \cdot\|f-\tilde{f}\|_{0}+M_{* *} \cdot\|c-\tilde{c}\|_{0}\right) .
\end{aligned}
$$

Now we can show the continuous dependence in the domain-restricted situation. Fix $0<\lambda<1$, choose a step size $0<h<1 / \kappa_{\lambda}$ and let $L:=\lfloor\lambda / h\rfloor \in \mathbb{N}$ be the number of steps. Furthermore, let

$$
\left(\left\|u_{0}-\tilde{u}_{0}\right\|_{L^{1}\left(\partial \Omega, \mathcal{H}^{1}\right)}+\mathcal{L}^{2}(\Omega) \cdot\|f-\tilde{f}\|_{0}+M_{* *} \cdot\|c-\tilde{c}\|_{0}\right) \leq \delta,
$$

for some $\delta>0$. Let $l \in \mathbb{N}_{0}, l \leq L$. With the result above we estimate on the set $\Omega_{T_{0}<(l+1) h}$ :

$$
\begin{gathered}
\|w\|_{L^{1}\left(\Omega_{T_{0}<(l+1) h}\right)} \leq C_{\lambda} \cdot \mathcal{L}^{2}(\Omega) \cdot\left(\lambda \cdot\|f[v]-f[\tilde{v}]\|_{L^{\infty}\left(\Omega_{T_{0}<l h}\right)}+h \cdot\|f[v]-f[\tilde{v}]\|_{L^{\infty}\left(\Omega_{T_{0}<(l+1) h}\right)}\right) \\
+C_{\lambda} \cdot M_{* *} \cdot\left(\lambda \cdot\|c[v]-c[\tilde{v}]\|_{L^{\infty}\left(\Omega_{T_{0}<l h}\right)}+h \cdot\|c[v]-c[\tilde{v}]\|_{L^{\infty}\left(\Omega_{T_{0}<(l+1) h}\right)}\right)+\lambda \cdot C_{\lambda} \cdot \delta .
\end{gathered}
$$


By using the Lipschitz condition on $c$ and $f$ and the definition of $\kappa_{\lambda}$ from Corollary 4.13 we obtain

$$
\|w\|_{L^{1}\left(\Omega_{T_{0}<(l+1) h}\right)} \leq \lambda \cdot C_{\lambda} \cdot \delta+\lambda \kappa_{\lambda} \cdot\|v-\tilde{v}\|_{L^{1}\left(\Omega_{T_{0}<l h}\right)}+h \kappa_{\lambda} \cdot\|v-\tilde{v}\|_{L^{1}\left(\Omega_{T_{0}<(l+1) h}\right)} .
$$

Now, we substitute the two fixed-points $u$ and $\tilde{u}$, i.e., we set $u_{1}=v=u$ and $u_{2}=\tilde{v}=\tilde{u}$,

$$
\|u-\tilde{u}\|_{L^{1}\left(\Omega_{T_{0}<(l+1) h}\right)} \leq \lambda \cdot C_{\lambda} \cdot \delta+\lambda \cdot \kappa_{\lambda} \cdot\|u-\tilde{u}\|_{L^{1}\left(\Omega_{T_{0}<l h}\right)}+h \cdot \kappa_{\lambda} \cdot\|u-\tilde{u}\|_{L^{1}\left(\Omega_{T_{0}<(l+1) h}\right)} \cdot
$$

We define the error on the set $\Omega_{T_{0}<l h}$ to be $e_{l}:=\|u-\tilde{u}\|_{L^{1}\left(\Omega_{T_{0}<l h}\right)}$. By our choice of $h$ we have $\left(1-h \kappa_{\lambda}\right)>0$ and estimate (4.15) yields an error recursion of the form

$$
e_{l+1} \leq \hat{\delta}+\alpha \cdot e_{l} \quad \Rightarrow \quad e_{l+1} \leq \sum_{k=0}^{l} \alpha^{k} \cdot \hat{\delta}
$$

where $\hat{\delta}:=\left(\lambda \cdot C_{\lambda} \cdot \delta\right) /\left(1-h \kappa_{\lambda}\right)$ and $\alpha:=\left(\lambda \cdot \kappa_{\lambda}\right) /\left(1-h \kappa_{\lambda}\right)$. On the restricted domain $\Omega_{T_{0}<\lambda}$ we have now $\|u-\tilde{u}\|_{L^{1}\left(\Omega_{T_{0}<\lambda}\right)} \leq e_{L+1} \leq C_{*} \cdot \delta$ and the continuous dependence is obvious in this case. Let now $\varepsilon>0$ be given. For the full domain $\Omega$ we choose $\lambda$ so close to 1 that $\| u-$ $\tilde{u} \|_{L^{1}\left(\Omega \backslash \Omega_{T_{0}<\lambda}\right)} \leq \varepsilon / 2$. Based on that $\lambda$ we find $h$ and $L$. What remains to do is to require $\delta=$ $C_{*}^{-1} \cdot \varepsilon / 2$, then $\|u-\tilde{u}\|_{L^{1}(\Omega)} \leq \varepsilon$ will hold whenever (4.14) holds.

5. Conclusions and Applications. In this paper we have demonstrated that the linear advection problem (3.1) on a two-dimensional compact domain with all inflow boundary can be well-posed. The solution which we construct is global and is defined on all of $\Omega$ as an element of the space $B V(\Omega)$; it satisfies the PDE on $\Omega \backslash \Sigma$ where $\Sigma$ is an interior outflow set. Secondly, we have shown that the corresponding quasi-linear functional problem (1.6), where we allow for quite general functional dependence, admits a solution but the solution need not be unique. To cope with that we restricted the problem to have causal Volterra-type functional dependence. Based on this causality we were able to prove uniqueness and as well as stability. As an application of the theory presented above we can derive the well-posedness of the PDE model behind Image Inpainting Based on Coherence Transport. What needs to be done here is to verify that the transport field $c$ of the inpainting model (1.1) satisfies Requirements 2.6 and 4.7. As pointed out in the introduction the set up of $c$ is complicated since $c$ is obtained from the vanishing-viscosity limit applied to the weight function of (1.5) which depends non-linearly on the structure tensor of (1.4). The verification of Requirements 2.6 and 4.7 is thus rather technical and is the subject of [23, Chapter 6]. Existence and uniqueness of the solution are then consequences of Theorems 4.5 and 4.14. We have also seen that the transport field $c$ depends on a couple of parameters, namely all the parameters of the structure tensor and the weight function, and on the data image $u_{0}$. Finally, as a consequence of Theorem 4.16 the solution of our inpainting model depends continuously on these parameters and $u_{0}$.

6. Acknowledgments. The author would like to thank Folkmar Bornemann and Colin Macdonald for their advice and the inspiring discussions.

\section{REFERENCES}

[1] T. Aach, C. Mota, I. Stuke, M. MÜHLICh, AND E. BARTh, Analysis of superimposed oriented patterns, IEEE Transactions on Image Proccessing, 15 (2006), pp. 3690-3700.

[2] C. Aliprantis AND K. BORder, Infinite Dimensional Analysis, Springer-Verlag, Berlin, 1994.

[3] H. AMANN, Ordinary Differential Equations: An Introduction to Nonlinear Analysis, de Gruyter, Berlin, 1990.

[4] L. Ambrosio, N. Fusco, AND D. Pallara, Functions of Bounded Variation and Free Discontinuity Problems, Oxford University Press, 2000.

[5] G. Aubert And P. Kornprobst, Mathematical Problems in Image Processing: Partial Differential Equations and the Calculus of Variations, vol. 147, Springer, 2006.

[6] C. Barnes, E. Shechtman, A. Finkelstein, And D. Goldman, PatchMatch: a randomized correspondence algorithm for structural image editing, ACM Transactions on Graphics-TOG, 28 (2009), p. 24. 
[7] M. Bertalmio, G. SAPIRo, V. Caselles, And C. BAllester, Image inpainting, in SIGGRAPH '00: Proceedings of the 27th Conference on Computer Graphics and Interactive Techniques, New Orleans, ACM Press/AddisonWesley Publishing Co., 2000, pp. 417-424.

[8] A. L. BertOzZI, S. ESEdOGLU, AND A. GiLlette, Analysis of a two-scale Cahn-Hilliard model for binary image inpainting, Multiscale Modeling \& Simulation, 6 (2007), pp. 913-936.

[9] - Inpainting of binary images using the Cahn-Hilliard equation, Image Processing, IEEE Transactions on, 16 (2007), pp. 285-291.

[10] F. BORNEMANN, Normen zur Charakterisierung der schwach*-Konvergenz beschränkter Folgen, Mathematische Semesterberichte, 48 (2002), pp. 247-260.

[11] F. BORNEMANN AND T. MÄRZ, Fast image inpainting based on coherence transport, Journal of Mathematical Imaging and Vision, 28 (2007), pp. 259-278.

[12] M. BURGER, L. HE, AND C.-B. SCHÖNLIEB, Cahn-Hilliard inpainting and a generalization for grayvalue images, SIAM Journal on Imaging Sciences, 2 (2009), pp. 1129-1167.

[13] F. CAO, Y. Gousseau, S. MASNOU, P. PÉREZ, ET AL., Geometrically guided exemplar-based inpainting, SIAM Journal on Imaging Sciences, 4 (2011), pp. 1143-1179.

[14] V. CASELles, J.-M. MOREL, AND C. SBERT, An axiomatic approach to image interpolation, IEEE Transactions on Image Processing, 7 (1998), pp. 376-386.

[15] T. ChAn AND J. SHEN, Image Processing and Analysis: Variational, PDE, Wavelet, and Stochastic Methods, Society for Industrial Mathematics, 2005.

[16] T. F. CHAN AND J. SHEN, Nontexture inpainting by curvature-driven diffusions, Journal of Visual Communication and Image Representation, 12 (2001), pp. 436-449.

[17] - Inpainting based on nonlinear transport and diffusion, in Inverse problems, Image Analysis, and Medical Imaging (New Orleans, LA, 2001), vol. 313 of Contemporary Mathematics, Providence, RI; American Mathematical Society, 2002, pp. 53-66.

[18] A. CRIMINISI, P. PÉREZ, AND K. TOYAMA, Region filling and object removal by exemplar-based image inpainting, IEEE Trans. Image Process., 13 (2004), pp. 1200-1212.

[19] K. DeIMLing, Nonlinear Functional Analysis, Springer, Berlin, 1985.

[20] G. GilboA AND S. OSHER, Nonlocal operators with applications to image processing, Multiscale Modeling \& Simulation, 7 (2008), pp. 1005-1028.

[21] H. Grossauer AND O. SCHERZER, Using the complex Ginzburg-Landau equation for digital inpainting in $2 d$ and $3 d$, in Scale Space Methods in Computer Vision, Springer, 2003, pp. 225-236.

[22] Z. KAmONT, Hyperbolic Functional Differential Inequalities and Applications, Kluwer Academic Publishers, 1999.

[23] T. MÄRZ, First Order Quasi-Linear PDEs with BV Boundary Data and Applications to Image Inpainting, Logos Verlag Berlin $\mathrm{GmbH}, 2010$.

[24] - Image inpainting based on coherence transport with adapted distance functions, SIAM Journal on Imaging Sciences, 4 (2011), pp. 981-1000.

[25] S. MASNOU, Disocclusion: a variational approach using level lines, IEEE Transactions on Image Processing, 11 (2002), pp. 68-76.

[26] S. MASNOU AND J.-M. MOREL, Level lines based disocclusion, in ICIP'98: Proceedings of the 1998 IEEE International Conference on Image Processing, Chicago, IEEE, 1998, pp. 259-263.

[27] P. Pérez, M. Gangnet, AND A. Blake, Patchworks: Example-based region tiling for image editing, Microsoft Research, Redmond, WA, Tech. Rep. MSR-TR-2004-04, (2004).

[28] J. SHEN, S. H. KANG, AND T. F. CHAN, Euler's elastica and curvature-based inpainting, SIAM Journal on Applied Mathematics, 63 (2003), pp. 564-592.

[29] J. SUN, L. YUAN, J. JIA, AND H. YEUNG SHUM, Image completion with structure propagation, in ACM Trans. Graphics, 2005, pp. 861-868.

[30] A. TELEA, An image inpainting technique based on the fast marching method, Journal of graphics tools, 9 (2004), pp. 2334.

[31] D. TSCHUMPERLÉ, Fast anisotropic smoothing of multi-valued images using curvature-preserving PDE's, International Journal of Computer Vision, 68 (2006), pp. 65-82.

[32] W. WALTER, Differential and Integral Inequalities, Springer, Berlin, 1970.

[33] J. WeICKERT, Anisotropic Diffusion in Image Processing, B.G. Teubner, Stuttgart, 1998.

[34] E. ZEIDLER, Fixed-Point Theorems, vol. 1 of Nonlinear Functional Analysis and its Applications, Springer Verlag, 1993. 



\section{RECENT REPORTS}

13/04 Spatially Partitioned Embedded Runge-Kutta Methods

Ketcheson

Macdonald

Ruuth

13/05 Simple computation of reaction-diffusion processes on point clouds

Macdonald

Merriman

Ruuth

13/06 A Volume-Based Method for Denoising on Curved Surfaces

Biddle

von Glehn

Macdonald

März

13/07 Porous squeeze-film flow

Knox

Wilson

Duffy

McKee

13/08 Diffusion of finite-size particles in confined geometries

Bruna

Chapman

13/09 Mathematical analysis of a model for the growth of the bovine corpus luteum

Prokopiou

Byrne

Jeffrey

Robinson

Mann

Owen

13/10 Capillary deformations of bendable films

Schroll

Adda-Bedia

Cerda

Huang

Menon

Russell

Toga

Vella

Davidovitch

13/11 Twist and stretch of helices: All you need is Love

Đuričković

Goriely

Maddocks

13/12 Switch on, switch off: stiction in nanoelectromechanical switches

Wagner

Vella

13/13 Pinning, de-pinning and re-pinning of a slowly varying rivulet

Paterson

Wilson

Duffy

13/14 Travelling-wave similarity solutions for a steadily translating slen- Yatim der dry patch in a thin fluid film

Duffy

Wilson

13/15 A stochastic model for early placental development

Cotter

Klika

Kimpton

Collins Heazel 
13/20 Complexity Plots

Thiyagalingam

Walton

Duffy

Trefethen

Chen

13/21 Glyph-based video visualization for semen analysis

Duffy

Thiyagalingam

Walton

Smith

Trefethen

Kirkman-Brown

Gaffney

Chen

13/22 RBF multiscale collocation for second order elliptic boundary

Farrell value problems

Wendland

13/23 $\mathrm{Na} / \mathrm{K}$ pump regulation of cardiac repolarization: Insights from a systems biology approach

Bueno-Orovio

Sánchez

Pueyo

Rodriguez

13/24 Cellular blebs - pressure-driven, axisymmetric, membrane protrusions

Woolley

Gaffney

Oliver

Baker

Waters

Goriely

13/25 Growth-induced axial buckling of a slender elastic filament em-

O'Keeffe bedded in an isotropic elastic matrix

Moulton

Waters

Goriely

13/26 The counterbend phenomenon: a generic property of the ax-

Gadêlha oneme and cross-linked filament bundles

Gaffney

Goriely

Copies of these, and any other OCCAM reports can be obtained from:

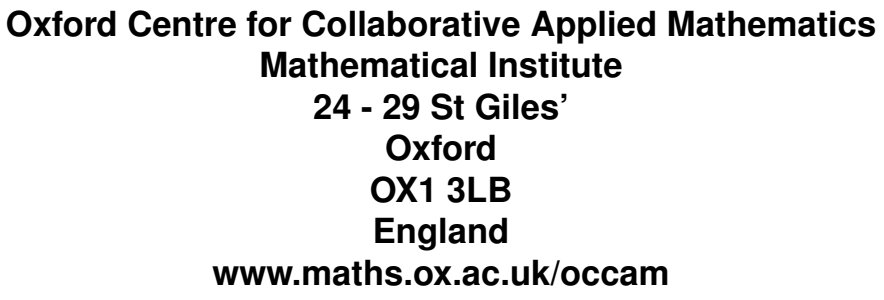

